\author{
И. И. Вульфсон \\ Т. С. Грибкова \\ М. В. Преображенская
}

\title{
ПРОГРАММНЫЕ ЗАКОНЫ ДВИЖЕНИЯ \\ РАБОЧИХ ОРГАНОВ ТЕХНОЛОГИЧЕСКИХ \\ МАШИН
}

Библиотека журнала «Вестник научно-технического развития»

The library of the Journal "Bulletin of Science and Technical Development"

(http://www.vntr.ru)

Представлено авторами

Публикуются в соответствии с решением редколлегии журнала

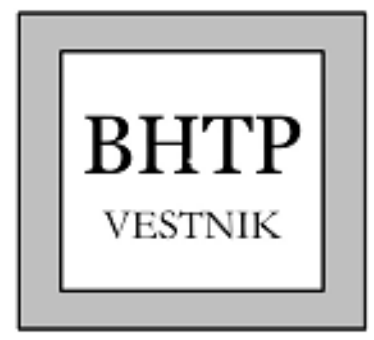

MOCKBA

2017 


\title{
ББК 4.41
}

B 88

Рецензенты:

Доктор технических наук, профессор СПГПУ В. Л. Жавнер Доктор технических наук, профессор СПГУПТД Л. С. Мазин

Вульфсон И. И., Грибкова.Т.С., Преображенская М. В. Программные законы движения технологических машин. / И. И. Вульфсон, Т. С. Грибкова, М.В. Преображенская — Библиотека ВНТР. - М.: ВНТР, 2017. - 108 с.

Книга даёт основные представления о современном подходе к решению важной инженерной задачи - синтеза закона движения рабочих органов при решении современных задач проектирования высокоскоростных машин с учетом технологических и динамических требований. Особое внимание уделено методам подавления возбуждаемых колебаний, которые являются основным источником искажений заданных программных движений. При решении оптимизационных задач использован аппарат безразмерных характеристических функций, облегчающий варьирование и оптимизацию параметров. Приведены соответствующие программы компьютерного моделирования, использование которых реализует рациональное совмещение аналитических и численных методов.

Книга существенно сокращает трудоемкость расчетов и может служить инструментом для инженеров- механиков. Она также может быть использована в качестве учебного пособия для студентов, магистров и слушателей факультетов повышения квалификации преподавателей соответствующих специальностей.

Печатается в авторской редакции.

Илл.: 52. Библиогр. 36 назв.

DOI:10.18411/a-2017-122

\author{
Библиотека журнала «Вестник Научно-Технического Развития» \\ (http://www.vntr.ru) \\ Опубликовано по решению редколлегии журнала. Представлено автором.
}

УДК $621+534.1$

ББК 34.41

(С) Вульфсон И. И., Грибкова.Т.С., Преображенская М. В.., 2017 


\section{ОГЛАВЛЕНИЕ}

ПРЕДИСЛОВИЕ 5

ГЛАВА 1. ЦИКЛОВЫЕ МЕХАНИЗМЫ....................................

1.1. Общие сведения о цикловых механизмах.................... 7

1.2. Программное движение звеньев цикловых механизмов........11

1.3. Простейшие критерии динамического синтеза................20

ГЛАВА 2. АНАЛИЗ ЗАКОНОВ ДВИЖЕНИЯ РАБОЧЕГО

ОРГАНА ПРИ УЧЕТЕ УПРУГОСТИ ЗВЕНЬЕВ

ЦИКЛОВОГО МЕХАНИЗМА $\ldots \ldots \ldots \ldots \ldots \ldots \ldots \ldots \ldots \ldots \ldots \ldots \ldots . .22$

2.1. Общее решение..............................................22

2.2. Вынужденные колебания при гармоническом возбуждении. 24

2.3. Вынужденные колебания при периодической функции

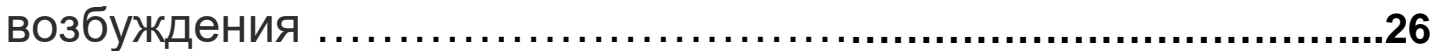

ГЛАВА 3. ВИБРОАКТИВНОСТЬ И ДИНАМИЧЕСКИЕ ОШИБКИ.............35

3.1. Оптимизация с помощью коррекции геометрических характеристик закона движения .............................35

3.2.Оптимизация с помощью коррекции частотных характеристик

3.3. Оптимизационный синтез законов движения с использованием компьютерного моделирования.

3.4. Динамические ошибки при кинематическом возбуждении исполнительных органов повышенной протяжённости........44

3.5. Минимизация ускорений на участках кратковременного реверса рабочих органов машин.

3.6. Инженерные рекомендации при синтезе законов движения кулачковых механизмов с учетом возбуждаемых колебаний....

3.7. Динамическая разгрузка...................................52

ГЛАВА 4. ВЛИЯНИЕ ЗАЗОРОВ НА ИСКАЖЕНИЯ ЗАКОНОВ

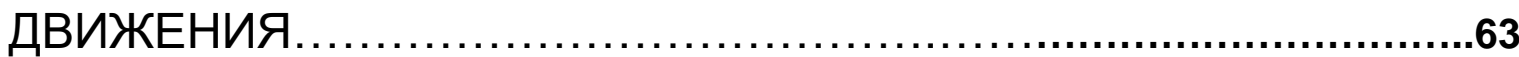

4.1. Динамические эфффекты ....................................63

4.2. Возбуждение колебаний при ударном характере взаимодействия элементов кинематических пар.....................65

4.3. Разрывы кинематической цепи и их устранение в механизмах с силовым замыканием ........................69

4.4. Продольные колебания замыкающих пружин..................72

4.5. Поперечные колебания замыкающих пружин................ 77 
ГЛАВА 5. СИНТЕЗ ЗАКОНОВ ДВИЖЕНИЯ РАБОЧЕГО ОРГАНА ПРИ УЧЕТЕ УПРУГОСТИ ПРИВОДА И ХАРАКТЕРИСТИК ЭЛЕКТРОДВИГАТЕЛЯ.

5.1. Линеаризация геометрических характеристик циклового механизма в окрестности программного движения..............82

5.2. Математическая модель. Условия устойчивости. .83

5.3. Колебания привода цикловой машины при учете динамической характеристики электродвигателя

5.4. Искажения программного движения в цикловых колебательных системах, вызванные двигателем

ГЛАВА 6. КОЛЕБАНИЯ КОМПЕНСАТОРА С ГИБКИМИ СВЯЗЯМИ ПРИ ПРОГРАММНОМ УПРАВЛЕНИИ НАТЯЖЕНИЕМ .95

6.1 Динамическая модель................................... 95

6.2. Условия устойчивости при совместном кинематическом и параметрическом возбуждении.

Список литературы. .101

Приложение .103 


\section{ПРЕДИСЛОВИЕ}

Данная книга посвящена одному из самых ответственных этапов проектирования механизмов - формированию закона движения выходного звена. За последние десятилетия этот этап претерпел существенные изменения качественного характера, что естественно нашло отражение в инженерных расчетах и учебном процессе. Если раньше при формировании законов движения доминировали расчётно-графические методы, то сейчас, с развитием компьютерной техники, синтез законов движения базируется на аналитических методах.

Одной из важных задач при реализации сложных законов программного движения рабочих органов машин является обеспечение высокой точности при выполнении технологических операций, что при постоянно растущих рабочих скоростях требует более глубокого учета динамических факторов. Поэтому современные представления о механике машин существенно отличаются от традиционного курса теории механизмов и машин. Это отличие, в первую очередь, состоит в учете упругодиссипативных характеристик звеньев и возбуждаемых колебаний.

Воспроизведение заданных программных законов движения осуществляется так называемыми цикловыми механизмами, среди которых можно выделить рычажные и кулачковые механизмы. Рычажные механизмы проще в изготовлении, однако, имеют ограниченные возможности при воспроизведении сложных законов движения. Даже такой, на первый взгляд, простой этап цикловой диаграммы машины, как выстой рабочего органа, нередко требует применения многозвенных рычажных механизмов, осуществляющих требуемое неподвижное в данном положении состояние ведомого звена лишь приближенно.

Учитывая вышеизложенные соображения, в качестве эталонного механизма принят кулачковый механизм, обладающий большими возможностями для удовлетворения повышенных требований к точности позиционирования рабочих органов при выполнении сложных технологических операций. При этом полученные результаты выходят за рамки проектирования кулачковых механизмов, так как определяют целевую направленность синтеза программных законов движения для любых механизмов и устройств при реализации этих законов. Следует также иметь в виду перспективность подобного расширенного подхода к рассматриваемой проблеме, если принять во внимание неизбежное развитие систем автоматического управления в этом классе задач.

Содержание книги базируется на научных публикациях и лекционном курсе проф. И.И. Вульфсона, отраженных в работах [5-14]. В настоящее время этот курс в сокращенной форме доступен широкому кругу читателей благодаря электронному варианту, помещенному в библиотеке журнала «Вестник научно-технического развития» (vntr.ru). Имея в виду это обстоятельство, некоторые подробности и доказательства в книге опущены (см. ссылки).

В данной книге используется ряд оригинальных компьютерных программ, в разработке которых основная роль принадлежит М.В. Преображенской [23 - 27]. Эти программы позволяют не только проиллюстрировать ис- 
следуемые режимы, но и успешно решать сложные оптимизационные задачи в интерактивном режиме. Программы прошли многолетнее апробирование и методическое совершенствование, проведенное Т.С. Грибковой.

Пособие состоит из шести глав. В первой главе введены понятия о функциях положения и геометрических передаточных функциях (аналогах скоростей и ускорений). Эти функции, предложенные в сороковых годах прошлого столетия профессорами Ленинградского политехнического института Х. Ф. Кетовым и Н. И. Колчиным, позволили отделить геометрические характеристики от кинематических $[1,14,20,22]$.

Следующий шаг в совершенствовании законов движения связан с введением безразмерных характеристик, в которых отражены основные свойства синтезируемого закона движения на участках разбега и выбега. При этом структурные параметры закона движения, связанные с конкретными технологическими и динамическими требованиями, играют роль масштабных факторов. Впервые безразмерные характеристики были предложены проф. К. В. Тиром как функции относительных значений перемещений ведомых звеньев и углов поворота ведущего звена [32]. В данном пособии используется другая безразмерная форма законов программного движения, с нашей точки зрения, более удобная для решения оптимизационных задач синтеза и компьютерного моделирования [6 - 8, 10, 13, 22].

Приведенная методика синтеза закона движения конкретизирована на примере трёх типовых задач, встречающихся в инженерной практике. Существенным отличием цикловых механизмов является трансформация равномерного вращения входного звена в неравномерное движение выходного звена. При этом нередко возникают большие динамические нагрузки, что особенно проявляется в высокоскоростных механизмах, которые одновременно являются источником колебаний и объектом виброзащиты. Этой проблеме посвящена основная часть книги.

Учёт дополнительных динамических требований при синтезе закона движения связан с возможностью варьирования параметрами, что позволяет осуществить оптимизацию, базируясь на важных динамических критериях. Последнее, в частности, находит отражение при выборе безразмерных характеристик с учётом упругости звеньев с целью уменьшить динамические ошибки, вызванные возбуждаемыми колебаниями. При этом многие традиционные представления об оптимальных законах движения потребовали существенного пересмотра. Так, в частности, переход от прямоугольного закона ускорения, который считался до середины XX века оптимальным, при учёте возбуждаемых колебаний оказался для высокоскоростных машин неприемлемым.

Книга даёт основные представления о современном подходе к решению важной инженерной задачи - синтеза закона движения рабочих органов и может служить инструментом для инженеров-механиков при решении современных задач проектирования технологических машин. Она также может быть использована в качестве учебного пособия для студентов, магистров и слушателей факультетов повышения квалификации преподавателей соответствующих специальностей. 


\section{ГЛАВА 1. ЦИКЛОВЫЕ МЕХАНИЗМЫ}

\section{1. Общие сведения о цикловых механизмах}

Функциональные особенности цикловых механизмов. Для формирования нелинейной функции положения выходных звеньев в машинах и автоматических линиях широко используются цикловые механизмы (рис. 1.1). Отличительной особенностью цикловых механизмов является нелинейность функции положения, трансформирующей координату на «входе» механизма в координату на «выходе». На рис 1.1 показаны наиболее распространенные разновидности простейших цикловых механизмов: рычажные (рис.1.1, $a, \sigma, 6)$, кулачковые (рис. 1, г) механизмы с некруглыми колесами (рис.1.1,д), шаговые, среди которых можно выделить мальтийские (рис.1.1, e), храповые (рис.1.1, ж) и червячные (рис. $1.1,3)$.

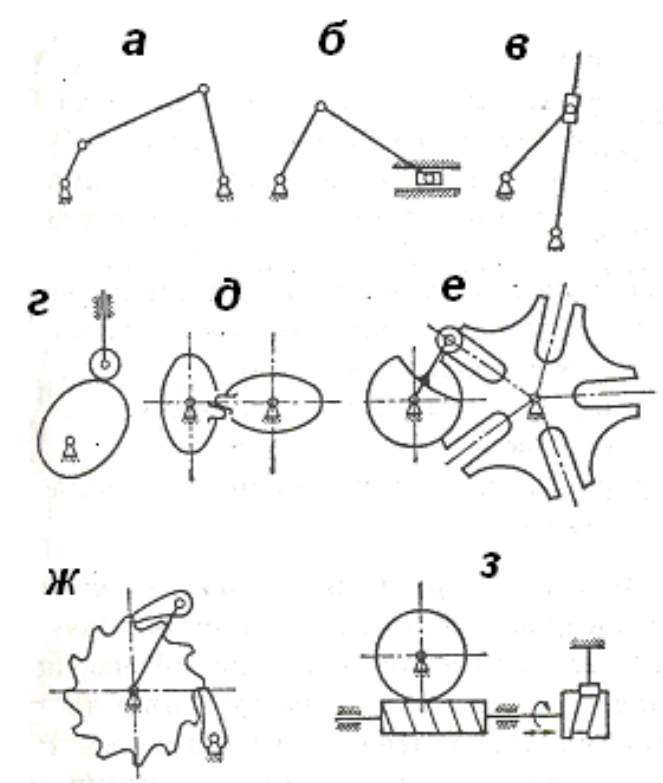

Рис. 1.1. Разновидности цикловых механизмов

Возможны различные сочетания указанных механизмов, например кулачково-рычажный, рычажно-шаговый, кулачково-шаговый и др. Кроме того, в соответствии с решаемой кинематической задачей эти простейшие механизмы могут быть значительно усложнены известным методом наслоения групп Aссура $[20,22,30,31]$. Иногда цикловой механизм шагового типа может быть создан на базе механизма с двумя степенями подвижности, осуществляющего сложение непрерывного равномерного вращательного движения с возвратнопоступательным или колебательным движениями. Пример схемы такого механизма, объединяющего свойства червячной передачи и кулачкового или рычажного механизмов, показан на рис. 1,3. В этом случае угловые перемещения червячного колеса, вызванные равномерным вращением червяка, суммируют- 
ся с дополнительными перемещениями от осевого возвратно-поступательного движения червяка, управляемого, например, кулачковым механизмом. Аналогичную задачу решает дифференциальный механизм, одно из ведущих колес которого вращается равномерно, а второе получает колебательное движение от кулачкового или рычажного механизма.

Все цикловые механизмы могут быть условно разделены на две группы - реверсивные и нереверсивные - в зависимости от того, равно или не равно нулю средняя скорость ведомого звена за период движения. В первом случае мы имеем возвратно-поступательное или колебательное движение звеньев около неподвижной оси (рис. $1, a, 6,6,2$ ), во втором - движение ведомого звена с отличной от нуля средней скоростью (рис. 1, $\partial, e$, ж, з), при котором в каждом цикле происходит смещение ведомого звена на один шаг.

Реализуемые в цикловых механизмах функции положения звеньев могут быть с выстоями (паузами) и без выстоев. По этому признаку различают механизмы прерывного и непрерывного движения. Кроме того, можно выделить квазипрерывное движение, для получения которого в современных машинах широко используются многозвенные рычажные механизмы с приближенным выстоем ведомого звена.

По своему функциональному назначению цикловые механизмы могут быть исполнительныли, передаточными, а также служить для управления, контроля, регулирования, питания, транспортировки, сортировки продукиии, автоматического счета изделий и m. n. Независимо от выполняемой операции каждый из этих механизмов может играть весьма ответственную роль в машине и подвергаться значительным динамическим нагрузкам. Поэтому с позиций динамического расчета деление механизмов по функциональному признаку обычно не является определяющим. Иногда с функциональным назначением механизма связаны некоторые особые требования к уровню допускаемых динамических искажений законов движения, динамических нагрузок и т. п., что должно быть учтено при синтезе механизма.

Кинематические и конструктивные особенности различных разновидностей цикловых механизмов подробно рассмотрены как в учебных пособиях по курсу теории механизмов и машин [22, 30, 31], так и в специальных монографиях.

Ниже излагается первый этап синтеза закона движения, основанный на рассмотрении так называемой идеальной кинетостатической модели, в которой исключены из рассмотрения зазоры и погрешности изготовления, а все звенья приняты в качестве абсолютно твердых тел. В последующих главах эти законы будут скорректированы с учетом упругости звеньев. Возможность варьирования законов программного движения вытекает из того обстоятельства, что кинематические требования к механизму обычно однозначно не обусловливают законов движения его звеньев и оставляют возможность их выбора по некоторым критериям динамического характера. Такая ситуация возникает, в частности, при решении задачи позиционирования, когда кинематические требования к механизму сводятся к перемещению выходного звена (рабочего органа) из данного начального в заданное конечное положение. 
Независимо от выполняемой операции эти механизмы, как правило, играют весьма ответственную роль в машине, поэтому к их надежности и точности предъявляются достаточно высокие требования. Трудности, возникающие при выполнении этих требований, связаны с тем, что при нелинейной функции положения динамические условия оказываются более напряженными, чем при линейной, из-за переменной скорости выходных звеньев цикловых механизмов. Это часто приводит к возникновению весьма значительных инерционных нагрузок. Кинематические требования, а следовательно, и связанные с ними динамические характеристики, могут быть реализованы в различных цикловых механизмах отнюдь не в равной степени. Например, в кулачковых механизмах профилированием рабочих поверхностей кулачков можно непосредственно подчинить движение выходного звена заданному закону движения. В рычажных же механизмах геометрические характеристики по существу заложены в их схеме, поэтому рациональным выбором конечного числа параметров можно лишь приблизиться к заданному эталону.

Если бы при сопоставлении динамических показателей цикловых механизмов мы основывались только на программных законах движения, не принимая во внимание возможности их практической реализации, то четко выявились бы преимущества кулачкового механизма, обладающего большими возможностями при синтезе для учета геометрически обусловленных динамических факторов. Однако во многих случаях важную роль играют динамические эффекты, вызванные ошибками изготовления и сборки механизма. Здесь приходится принимать во внимание, что рабочие поверхности элементов низших кинематических пар, используемых в рычажных механизмах, весьма просты и по сравнению со сложными профилями кулачков могут быть изготовлены точнее. С другой стороны, сложные законы движения, осуществляемые с помощью кулачковых механизмов исключительно просто, при применении рычажных механизмов обычно могут быть реализованы лишь при большом числе звеньев. При этом растут массы, габариты, зазоры, что в целом отрицательно сказывается на динамике механизма. Итак, не конкретизируя задачу, можно утверждать лишь одно: чем проще закон движения, тем более ощутимы преимущества рычажных механизмов перед кулачковыми.

Поскольку с помощью кулачковых механизмов закон программного движения может быть принципиально воспроизведен теоретически точно, при дальнейшем изложении будем ориентироваться именно на этот класс механизмов. Полученные при этом законы движения могут быть использованы в качестве эталонов для приближенного метрического синтеза рычажных механизмов, а также при решении задачи позиционирования рабочих органов средствами программного управления .

Движения исполнительных органов, обеспечивающие выполнение заданных технологических или транспортных операций, называют программными. Программные движения оказывают существенное влияние на уровень возбуждаемых колебаний, поэтому задача снижения виброактивности машин тесно соприкасается с проблемой формирования оптимальных законов движения. 
Функция положсеня и геометрические передаточные функции. Будем понимать под идеальным механизмом его кинетостатическую модель при абсолютно точном воспроизведении заданных характеристик, т.е. такой абстрактный механизм, в котором звенья не деформируются, отсутствуют зазоры и погрешности изготовления. Если такой механизм имеет одну степень подвижности, то положение любого звена механизма однозначно определяется в зависимости от угла поворота входного звена $\varphi_{1}$. Для определенности примем, что звено $n$ совершает вращательное или поступательное движение, описываемое одной координатой $\varphi_{n}$. Тогда

$$
\varphi_{n}=\Pi_{n}\left(\varphi_{1}\right),
$$

где $\Pi_{n}-$ функция положения звена $n$.

Рассмотрим следующие функции, полученные дифференцированием

$$
\Pi_{n}^{\prime}=\frac{d \Pi_{n}}{d \varphi_{1}} ; \Pi_{n}^{\prime \prime}=\frac{d^{2} \Pi_{n}}{d \varphi_{1}^{2}} ; \Pi_{n}^{\prime \prime \prime}=\frac{d^{3} \Pi_{n}}{d \varphi_{1}^{3}},
$$

которые называются соответственно первой, второй и третьей геометрическими передаточными функииями, либо аналогами скоростей, ускорений и ускорений второго порядка $[1,6-10,17,18,22,28]$,. Если $\varphi_{1}$ отвечает угловой координате, то размерность передаточных функций совпадает с размерностью $\Pi_{n}$.

Плоскопараллельное движение звена может быть описано тремя функциями положения, фиксирующими угловую координату звена и положение одной из его точек. Связь геометрических характеристик $\Pi_{n}^{\prime}$, $\Pi_{n}^{\prime \prime}$, $\Pi_{n}^{\prime \prime \prime}$ с кинематическими $\dot{\varphi}_{n}=d \varphi_{n} / d t ; \ddot{\varphi}_{n}=d^{2} \varphi_{n} / d t^{2} ; \dddot{\varphi}_{n}=d^{3} \varphi_{n} / d t^{3}$ определяется следующими зависимостями:

$$
\left.\begin{array}{l}
\dot{\varphi}_{n}=\Pi_{n}^{\prime}\left(\varphi_{1}\right) \dot{\varphi}_{1} ; \\
\ddot{\varphi}_{n}=\Pi_{n}^{\prime \prime}\left(\varphi_{1}\right) \dot{\varphi}_{1}^{2}+\Pi_{n}^{\prime}\left(\varphi_{1}\right) \ddot{\varphi}_{1} ; \\
\dddot{\varphi}_{n}=\Pi_{n}^{\prime \prime \prime}\left(\varphi_{1}\right) \dot{\varphi}_{1}^{3}+3 \Pi_{n}^{\prime \prime}\left(\varphi_{1}\right) \dot{\varphi}_{1} \ddot{\varphi}_{1}+\Pi_{n}^{\prime}\left(\varphi_{1}\right) \dddot{\varphi}_{1} .
\end{array}\right\}
$$

Структура выражений (1.2) свидетельствует о том, что при использовании передаточных функций имеет место четкое разделение геометрических и кинематических характеристик, описывающих движение рассматриваемого звена механизма. В частном случае, в зубчатых механизмах с постоянным передаточным отношением функция положения линейна. Как следует из зависимостей (1.2), в этом случае $\dot{\varphi}_{n}=\Pi_{n}^{\prime} \dot{\varphi}_{1} ; \ddot{\varphi}_{n}=\Pi_{n}^{\prime} \ddot{\varphi}_{1} ; \dddot{\varphi}_{n}=\Pi_{n}^{\prime} \dddot{\varphi}_{1}$, причем коэффициентом пропорциональности здесь служит первая передаточная функция. Если к тому же входное звено движется с постоянной скоростью $\dot{\varphi}_{1}=$ const, то и выходное звено будет перемещаться равномерно. Следовательно, возникновение инерционных нагрузок в подобных механизмах возможно только за счет 
нарушения условий $\dot{\varphi}_{1}=$ const или $\Pi_{n}^{\prime}=$ const из-за ошибок при изготовлении и других погрешностей.

\section{2. Программное движение звеньев цикловых механизмов}

Способы получения программных движений. В современных машинах используются два способа формирования законов движения звеньев.

Первый способ широко применяется в цикловых технологических и энергетических машинах, осуществляющих свои функции при установившемся режиме работы, когда скорость двигателя $\omega$, после некоторого переходного режима достигает примерно постоянного значения. Для реализации заданных законов движения используются так называемые цикловые механизмы (рычажные, кулачковые, мальтийские и др.), с помощью которых осуществляется нелинейное преобразование координаты на «входе» $\varphi=\omega t$ в соответствующую координату на «выходе».

При использовании второго способа формирование заданных программных движений обеспечивается с помощью программного управления серводвигателей (так называемые, «электронные кулачки»). В подобных случаях механическая система машины обычно имеет более простую структуру, поскольку механизмы осуществляют только линейное преобразование координат, как это имеет место, например, в зубчатых передачах с постоянным передаточным отношением. Другими несомненными преимуществами данного способа являются большая гибкость при настройке, уменьшение масс и моментов инерции, а, следовательно, и динамических нагрузок, снижение габаритов конструкции и др. Типичными примерами использования электронных кулачков являются современные упаковочные, полиграфические, некоторые текстильные машины, сборочные автоматы, деревообрабатывающие станки и др. В то же время широкое использование этого способа затруднено в тех случаях, когда технологический процесс или транспортная операция требует точной цикловой синхронизации с другими исполнительными органами. Подобная задача в скоростных машинах пока более надёжно решается посредством установки входных звеньев цикловых механизмов на достаточно жёстком распределительном валу.

Нередко задача программного управления решается человекомоператором, например, при управлении транспортными машинами (автомобилями, подъемными кранами, некоторыми видами промышленных роботов и др.)

Ниже мы в основном ограничимся анализом динамических процессов, реализуемых непосредственно в механической системе.

Структура закона движения. Безразмерные характеристики. Независимо от специфических требований, предъявляемых к цикловому механизму, и его функционального назначения в конкретной машине он должен удовлетворять ряду общих динамических условий. Чаще всего - это требование 
плавности движения, исключающее возможность нарушения непрерывности функции положения П и первой геометрической передаточной функции П' При этом достаточно общей оказывается трехпериодная структура интервала движения, при которой перемещение выходного звена в одном направлении (прямой или обратный ход) рассматривается как совокупность трех участков (рис. 1.2): разбега, равномерного движения 2 и выбега 3. (В целях упрощения записи индексы при геометрических характеристиках, указывающие номер звена, в дальнейшем будут опущены.)

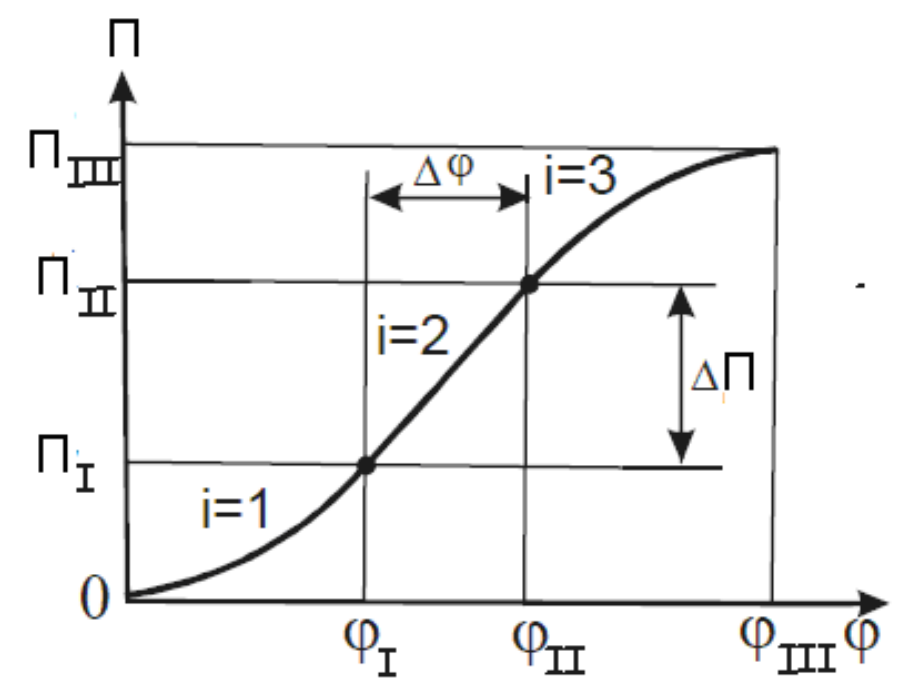

Рис.1.2 . График функции положения

При синтезе законов движения целесообразно воспользоваться аппаратом безразмерных характеристик. Введем в рассмотрение следующие функции:

$$
\begin{gathered}
\frac{\varphi}{\varphi_{1}}=\tau_{1} ; \frac{\Pi}{\Pi_{1}}=\theta_{1}\left(\tau_{1}\right) \text { ï ðѐ } \varphi \in\left[0, \varphi_{1}\right] ; \\
\frac{\varphi_{111}-\varphi}{\varphi_{111}-\varphi_{11}}=\tau_{3} ; \frac{\Pi_{111}-\Pi}{\Pi_{111}-\Pi_{11}}=\theta_{3}\left(\tau_{3}\right) \text { ï ðè } \varphi \in\left[\varphi_{11}, \varphi_{11}\right] .
\end{gathered}
$$

Функции $\tau_{1}=0, \quad \theta_{1}=0$ при $\quad \varphi=\varphi_{1} ; \quad \tau_{3}=1, \quad \theta_{3}=1$ при $\varphi=\varphi_{11} ; \quad \tau_{3}=0, \quad \theta_{3}=0$ при $\varphi=\varphi_{111}$. Таким образом, графики безразмерных характеристик $\theta_{1}\left(\tau_{1}\right)$ и $\theta_{3}\left(\tau_{3}\right)$ вписываются в квадрат со сторонами, равными единице. Если на разбеге и выбеге принят один и тот же тип закона движения, то функции $\theta_{1}$ и $\theta_{3}$ совпадают. Функции положения и геометрические передаточные функции, выраженные через безразмерные характеристики, приведены в табл. 1.1.

Очевидно, что изменением функций $\Pi, \quad \Pi^{\prime}=d \Pi / d \varphi, \quad \Pi^{\prime \prime}=d^{2} \Pi / d \varphi^{2}$ управляют функции $\theta_{i}, \quad \theta_{i}^{\prime}=d \theta_{i} / d \tau_{i}, \quad \theta_{i}^{\prime \prime}=d^{2} \theta_{i} / d \tau_{i}^{2}$. Остальные параметры играют роль масштабных факторов. Таким образом, если введением геометрических передаточных функций было произведено разделение геометрических и 
кинематических факторов, то при введении безразмерных характеристик из передаточной функции оказались выделенными масштабные факторы $\varphi_{1}, \varphi_{11}, \varphi_{111}, \Pi_{1}, \Pi_{11}, \Pi_{111}$, с помощью которых принятые безразмерные характеристики закона движения «деформируются» по осям ф и П. В дальнейшем эти масштабные факторы будем называть структурными параметрами закона движения.

Таблица 1.1. Функции положения и геометрические передаточные функции

\begin{tabular}{|l|c|c|c|}
\hline $\begin{array}{l}\text { Функ } \\
\text { ция }\end{array}$ & Разбег & Участок постоянной скорости & Выбег \\
\hline$\Pi$ & $\Pi_{\mathrm{I}} \theta_{1}\left(\tau_{1}\right)$ & $\Pi_{\mathrm{I}}+\frac{\Pi_{\mathrm{II}}-\Pi_{\mathrm{I}}}{\varphi_{\mathrm{II}}-\varphi_{\mathrm{I}}}\left(\varphi-\varphi_{\mathrm{I}}\right)$ & $\Pi_{\mathrm{III}}-\left(\Pi_{\mathrm{III}}-\Pi_{\mathrm{II}}\right) \theta_{3}\left(\tau_{1}\right)$ \\
\hline$\Pi^{\prime}$ & $\frac{\Pi_{\mathrm{I}}}{\varphi_{\mathrm{I}}} \theta^{\prime}\left(\tau_{1}\right)$ & $\frac{\Pi_{\mathrm{II}}-\Pi_{\mathrm{I}}}{\varphi_{\mathrm{II}}-\varphi_{\mathrm{I}}}$ & $\frac{\Pi_{\mathrm{III}}-\Pi_{\mathrm{II}}}{\varphi_{\mathrm{III}}-\varphi_{\mathrm{II}}} \theta_{3}^{\prime}\left(\tau_{3}\right)$ \\
\hline$\Pi^{\prime \prime}$ & $\frac{\Pi_{\mathrm{I}}}{\varphi_{\mathrm{I}}^{2}} \theta^{\prime \prime}\left(\tau_{1}\right)$ & 0 & $-\frac{\Pi_{\mathrm{II}}-\Pi_{\mathrm{II}}}{\left(\varphi_{\mathrm{III}}-\varphi_{\mathrm{II}}\right)^{2}} \theta_{3}^{\prime \prime}\left(\tau_{3}\right)$ \\
\hline
\end{tabular}

Для исключения ударов в начале и конце хода потребуем $\Pi^{\prime}(0)=0$ и $\Pi^{\prime}\left(\varphi_{111}\right)=0$, а следовательно $\theta_{i}^{\prime}(0)=0$ и $\Pi^{\prime}\left(\varphi_{111}\right)=0 \quad(i=1 ; 3)$. При $\tau_{i}=1$ функция $\theta_{i}^{\prime}\left(\tau_{i}\right)$ достигает своего максимального значения $\theta_{\max }^{\prime}$. Легко убедиться, что константа $\theta_{\max }^{\prime}$ показывает, во сколько раз максимальная скорость на рассматриваемом участке выше средней скорости. Функция $\theta_{i}^{\prime \prime}\left(\tau_{i}\right)$ в зависимости от выбранного закона движения может достичь своего максимального значения при различных значениях $\tau_{i}$. Отношение $\theta_{\max }^{\prime \prime} / \theta_{\max }^{\prime}$ показывает, во сколько раз максимальное ускорение на данном участке выше среднего значения.

Проекции боковых сторон определяются параметрами $s_{1}$ и $s_{2}$, которыми можно эффективно управлять законом программного движения. При $s_{1}=0$ и $s_{2}=0$ имеем так называемый прямоугольный закон ускорений; при $s_{1}=s_{2}=0,5$ - синусоидальный; при $s_{1}=0, s_{2}=1$ - косинусоидальный. Большое распространение получил закон равнобокой трапеции при $s_{1}=s_{2}=0,25$ (подробнее о влиянии параметров $s_{1}, s_{2}$ cм. п. 3.1).

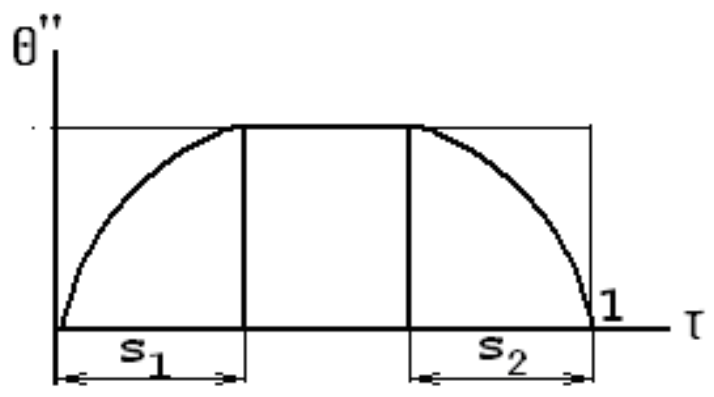

Рис.1.3. График безразмерной характеристики $\theta^{\prime \prime}(\tau)$ 
В табл. 1.2 приведены расчетные зависимости и константы, для распространенного в инженерной практике семейства безразмерных характеристик, известного под названием «модифицированная трапеция общего вида». Для этого типа закона движения график функции $\theta^{\prime \prime}(\tau)$ представляет собой трапецию, у которой боковые стороны образованы отрезками синусоиды (рис.1.3).

Таблица 1.2. Безразмерные характеристические функции закона движеНия

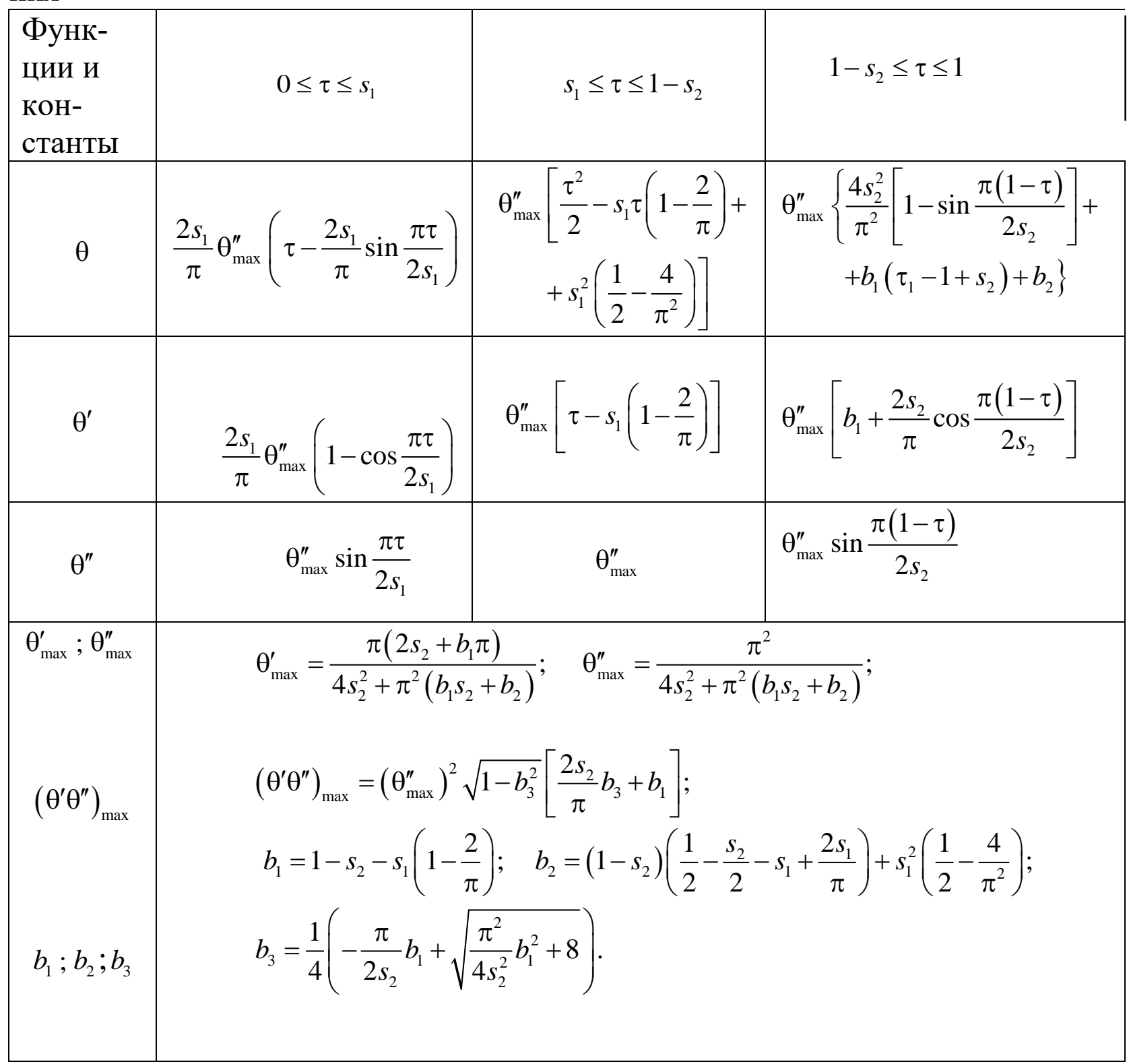

Из всех возможных законов самым малым значением $\theta_{\max }^{\prime}=2$ обладает прямоугольный закон ускорений. Однако при этом законе ускорения имеются разрывы непрерывности (мягкие удары), что приводит к возбуждению интенсивных колебаний (см. гл.2). Однако не всякий скачок, заложенный в функции $\theta^{\prime \prime}$, обязательно приводит к мягкому удару. Например, если толкатель кулачного механизма перемещается без выстоя, то можно на границе прямого и об- 
ратного ходов «застыковать» ускорения без скачка, не требуя, чтобы в точке стыкования ускорения были равны нулю. Окончательное суждение о приемлемости и достоинствах того или иного закона движения должно основываться на учете характеристик конкретной колебательной системы.

\section{Общие свойства безразмерных констант законов движения}

С в о й с т в о 1 . Константа $\theta_{\max }^{\prime}$ обратно пропорциональна величине $1-\tau_{*}$, где $\tau_{*}$-абсцисса центра тяжести площади фигуры, ограниченной графиком $\theta^{\prime \prime}(\tau)$ и осью абсцисс (см. рис.1.3). Для доказательства этого положения найдем $\tau_{*}$ :

$$
\tau_{*}=\frac{\int_{0}^{1} \tau \theta^{\prime \prime}(\tau) d \tau}{\int_{0}^{1} \theta^{\prime \prime}(\tau) d \tau}=\frac{\theta_{\max }^{\prime}-1}{\theta_{\max }^{\prime}} .
$$

Из (1.4) следует

$$
\theta_{\max }^{\prime}=1 /\left(1-\tau_{*}\right) .
$$

Очевидно, что для всех симметричных эпюр $\theta^{\prime}(\tau) \tau_{*}=0,5$, а следовательно, $\theta_{\max }^{\prime}=2$.

С в о й с т в о 2. Константа $\theta_{\max }^{\prime \prime}$ прямо пропорциональна константе $\theta_{\max }^{\prime}$ и обратно пропорциональна коэффициенту заполнения $\sigma$. Под коэффициентом заполнения $\sigma$ будем понимать отношение площади фигуры, ограниченной графиком $\theta_{\max }^{\prime \prime}$ и осью абсцисс, к площади описанного прямоугольника (см. рис. 1.3). Итак,

$$
\sigma=\int_{0}^{1} \theta^{\prime \prime} d \tau / \theta_{\max }^{\prime \prime}=\theta_{\max }^{\prime} / \theta_{\max }^{\prime \prime}
$$

Отсюда следует

$$
\theta_{\max }^{\prime \prime}=\theta_{\max }^{\prime} / \sigma=\left(1-\tau_{*}\right)^{-1} \sigma^{-1} .
$$

Поскольку $\sigma_{\max }=1$, минимальное значение $\theta_{\max }^{\prime \prime}=2$ реализуется при прямоугольном законе изменения ускорений. Область существования решений соответствует очевидным ограничениям $\sigma \leq 1, \quad \theta_{\max }^{\prime}>1, \quad \theta_{\max }^{\prime \prime} \geq 2$.

На данном этапе будем считать функции $\theta(\tau)$ заданными. К вопросам, связанным с рациональным выбором безразмерных характеристик, мы еще вернемся в п.1.3 и в гл.3 .

Определение структурных параметров для типовых задач синтеза закона движения. Перечисленные выше шесть параметров не могут быть заданы произвольно, так как они во избежание ударов должны быть связаны двумя условиями непрерывности первой геометрической передаточной функции $\Pi^{\prime \prime}$ на границах участков, т. е. при $\varphi=\varphi_{1}$ и $\varphi=\varphi_{11}$ : 


$$
\begin{aligned}
& \frac{\Pi_{\mathrm{I}}}{\varphi_{\mathrm{I}}} \theta_{1 \max }^{\prime}=\frac{\Pi_{\mathrm{II}}-\Pi_{\mathrm{I}}}{\varphi_{\mathrm{II}}-\varphi_{\mathrm{I}}} ; \\
& \frac{\Pi_{\mathrm{II}}-\Pi_{\mathrm{I}}}{\varphi_{\text {II }}-\varphi_{\mathrm{I}}}=\frac{\Pi_{\mathrm{III}}-\Pi_{\mathrm{II}}}{\varphi_{\mathrm{III}}-\varphi_{\mathrm{II}}} \theta_{3 \max }^{\prime} .
\end{aligned}
$$

При $\varphi=0$ и $\varphi=\varphi_{\text {III }}$ аналогичные условия удовлетворяются тем, что $\theta_{i}^{\prime}(0)=0$. Таким образом, для однозначного решения задачи синтеза закона движения кроме безразмерных характеристик следует задать четыре дополнительных условия, исходя из конкретных условий.

Рассмотрим ряд типовых задач синтеза законов программного движения. Предварительно введем несколько безразмерных параметров, характеризующих относительную величину участка постоянной скорости:

$$
\zeta_{n}=\left(\Pi_{\text {II }}-\Pi_{\mathrm{I}}\right) / \Pi_{\mathrm{III}} ; \zeta_{\varphi}=\left(\varphi_{\mathrm{II}}-\varphi_{\mathrm{I}}\right) / \varphi_{\mathrm{III}},
$$

a также коэффициент асимметрии закона движения

$$
f=\left(\varphi_{\text {III }}-\varphi_{\text {II }}\right) / \varphi_{\text {I. }} \text {. }
$$

При $f=1$ длительности разбега и выбега равны.

Задача 1. Дано: $\Pi_{\text {III }}, \varphi_{\text {III }}, f, \zeta_{n}$. получаем

На основании (1.3) при учете (1.4) и (1.5) после элементарных выкладок

$$
\begin{aligned}
& \Pi_{\mathrm{I}}=\Pi_{\mathrm{III}}\left(1-\zeta_{n}\right) /\left(1+v_{1} f\right) ; \varphi_{\mathrm{I}}=\varphi_{\mathrm{III}}\left(1-\zeta_{\varphi}\right) /(1+f) ; \\
& \Pi_{\mathrm{II}}=\Pi_{\mathrm{III}}\left(1+v_{1} f \zeta_{n}\right) /\left(1+v_{1} f\right) ; \varphi_{\mathrm{II}}=\varphi_{\mathrm{III}}\left(1+f \zeta_{\varphi}\right) /(1+f),
\end{aligned}
$$

где $v_{1}=\theta_{1 \max }^{\prime} / \theta_{3 \max }^{\prime}$.

Теперь остается определить неизвестный параметр $\zeta_{\varphi}$. После подстановки (1.10) в (1.8) запишем

$$
\frac{\zeta_{n}}{\zeta_{\varphi}}=\frac{\left(1-\zeta_{n}\right)(1+f)}{\left(1+v_{1} f\right)\left(1-\zeta_{\varphi}\right)} \theta_{1 \max }^{\prime}
$$

Решая это уравнение относительно $\zeta_{\varphi}$, имеем

$$
\zeta_{\varphi}=\frac{\zeta_{n}}{\zeta_{n}+U\left(1-\zeta_{n} \theta_{1 \max }^{\prime}\right)}
$$

где $U=(1+f) /\left(1+v_{1} f\right)$.

Если на разбеге и выбеге принят один и тот же тип закона движения, то $\theta_{1 \max }^{\prime}=\theta_{3 \max }^{\prime}, \quad v_{1}=1, \quad$ а следовательно, $U=1$. Выражения (1.10) и (1.11) однозначно определяют решение задачи. Если вместо параметра $\zeta_{n}$ задан $\zeta_{\varphi}$, уравнение (1.11) следует решить относительно $\zeta_{n}$.

Конкретизируем для рассматриваемой задачи простейшие динамические критерии, приведенные в начале этого параграфа:

$$
\Pi_{\max }^{\prime}=\frac{\Pi_{\mathrm{I}}}{\varphi_{\mathrm{I}}} \theta_{1 \text { max }}^{\prime}=\frac{\Pi_{\mathrm{III}}-\Pi_{\mathrm{II}}}{\varphi_{\mathrm{III}}-\varphi_{\mathrm{II}}} \theta_{3 \max }^{\prime} ;
$$

для разбега 


$$
\Pi_{\max }^{\prime \prime}=\Pi_{\max }^{\prime \prime}=\frac{\Pi_{\mathrm{I}}}{\varphi_{\mathrm{I}}^{2}} \theta_{1 \text { max }}^{\prime \prime} ; \quad\left(\Pi^{\prime} \Pi^{\prime \prime}\right)_{\max }=\frac{\Pi_{\mathrm{I}}^{2}}{\varphi_{\mathrm{I}}^{3}}\left(\theta_{1}^{\prime} \theta_{1}^{\prime \prime}\right)_{\max } ;
$$

для выбега

$$
\left|\Pi^{\prime \prime}\right|_{\max }=\frac{\Pi_{\text {III }}-\Pi_{\text {II }}}{\left(\varphi_{\text {III }}-\varphi_{\text {II }}\right)^{2}} \theta_{3 \text { max }}^{\prime \prime} ;\left|\Pi^{\prime} \Pi^{\prime \prime}\right|_{\max }=\frac{\left(\Pi_{\text {III }}-\Pi_{\text {II }}\right)^{2}}{\left(\varphi_{\text {III }}-\varphi_{\text {II }}\right)^{3}}\left(\theta_{3}^{\prime} \theta^{\prime \prime}\right)_{3 \max } \cdot
$$

Согласно формулам (1.7) структурные параметры в общем случае также зависят от констант безразмерных характеристик $\theta_{1 \max }^{\prime}, \theta_{3 \max }^{\prime}$, поэтому характер их влияния на данные критерии отнюдь не столь очевиден, как это следует формально из выражений (1.12) - (1.14). Лишь в самом простом случае, когда отсутствует участок постоянной скорости $\left(\zeta_{n}=\zeta_{\varphi}=0\right)$ и $\theta_{1 \max }^{\prime}=\theta_{3 \max }^{\prime}\left(v_{1}=1\right)$, получаем, что рассматриваемые критерии пропорциональны соответствующим безразмерным константам.

С увеличением участка постоянной скорости значение $\Pi_{\max }^{\prime \prime}$ убывает, a $\left|\Pi_{\max }^{\prime \prime}\right|$ обычно растет. В предельном случае, когда $\varphi_{1}=0, \quad \varphi_{\text {II }}=\varphi_{\text {III }}\left(\zeta_{n}=\zeta_{\varphi}=1\right)$ имеем $\min \Pi_{\max }^{\prime}=\Pi_{\mathrm{III}} / \varphi_{\mathrm{III}}$; остальные критерии неограниченно возрастают. Встречный характер влияния $\zeta_{n}$ (или $\zeta_{\varphi}$ ) на $\Pi_{\max }^{\prime}$ и $\left|\Pi_{\max }^{\prime \prime}\right|$ свидетельствует о том, что при определенном значении участка постоянной скорости, отличном от нуля, имеет место минимум критерия $\left|\Pi^{\prime} \Pi^{\prime \prime}\right|_{\max }$, который пропорционален динамической составляющей движущего момента.

Проиллюстрируем это на примере, в котором примем законы движения на разбеге и выбеге одинаковыми, а графики $\theta_{1,3 \max }^{\prime}\left(\tau_{1,3}\right)-$ симметричными $\left(\tau_{\ddot{0}}=0,5\right)$; при этом $f=1, \theta_{\max }^{\prime}=2, \quad v_{1}=1$.

На основании (1.7) имеем $\zeta_{\varphi}=\zeta_{n} /\left(2-\zeta_{n}\right)$. Тогда на разбеге и выбеге

$$
\left|\Pi^{\prime} \Pi^{\prime \prime}\right|_{\max }=\frac{\Pi_{\mathrm{III}}^{2}\left(2-\zeta_{n}\right)^{3}}{8 \varphi_{\mathrm{III}}^{3}\left(1-\zeta_{n}\right)}\left(\theta^{\prime} \theta^{\prime \prime}\right)_{\max } .
$$

Легко убедиться в том, что минимуму этой функции при варьировании параметра $\zeta_{n}$ отвечает значение $\zeta_{n}=1 / 2$; при этом $\zeta_{\varphi}=1 / 3$. Подстановка этих значений в (1.12) - (1.14) показывает, что за счет введения участка постоянной скорости величина $\left|\Pi^{\prime} \Pi^{\prime \prime}\right|_{\max }$ понизилась на $15,6 \%, \quad \Pi_{\max }^{\prime}$ — на $25 \%$ при увеличении $\left|\Pi^{\prime \prime}\right|_{\max }$ на $12,5 \%$.

Характер влияния коэффициента асимметрии $f$ будет проанализирован при рассмотрении следующей задачи.

3 а д а ч а 2. Дано: $\Pi_{\mathrm{III}}, \varphi_{\mathrm{III}}, \zeta_{n}$ (или $\left.\zeta_{\varphi}\right), \rho=\Pi_{\mathrm{Imax}}^{\prime \prime} /\left|\Pi_{3}^{\prime \prime}\right|_{\max }$. Запишем отношение экстремальных значений ускорений на разбеге и выбеге (см. табл. 1.1):

$$
\rho=\frac{\Pi_{\mathrm{I}}\left(\varphi_{\mathrm{III}}-\varphi_{\mathrm{II}}\right)^{2}}{\left(\Pi_{\mathrm{III}}-\Pi_{\mathrm{II}}\right) \varphi_{\mathrm{I}}^{2}} v_{2}
$$

где $v_{2}=\theta_{1 \max }^{\prime \prime} / \theta_{3 \max }^{\prime \prime}$. 
Согласно (1.13), (1.14), имеем $\left(\varphi_{\mathrm{III}}-\varphi_{\mathrm{II}}\right)^{2} / \varphi_{\mathrm{I}}^{2}=f^{2}, \quad\left(\Pi_{\mathrm{III}}-\Pi_{\mathrm{II}}\right) / \Pi_{\mathrm{I}}=f v_{1}$. После подстановки в (1.15)

$$
\rho=f v_{2} / v_{1} .
$$

Итак, параметром $\rho$ однозначно определяется коэффициент асимметрии $f=\rho v_{1} / v_{2}$. Тем самым, эта задача сведена к условиям предыдущей. Зависимость (1.16) облегчает анализ влияния параметра $f$ на $|\Pi "|_{\max }$. При учете (1.13), (1.14), (1.16) имеем

$$
\Pi_{\mathrm{I} \max }^{\prime \prime}=\frac{\Pi_{\mathrm{III}}\left(1-\zeta_{n}\right)(1+f) U \theta_{1 \max }^{\prime}}{\left(1-\zeta_{\varphi}\right)^{2}} ; \quad\left|\Pi_{3}^{\prime \prime}\right|_{\max }=\frac{v_{1} \Pi_{I \max }^{\prime \prime}}{f v_{2}},
$$

где $U=(1+f) /\left(1+v_{1} f\right)$.

С увеличением параметра $f$ экстремальное значение второй передаточной функции на разбеге растет, а на выбеге убывает. Для выбора оптимального значения этого параметра можно воспользоваться условием минимума критерия $K_{1}$ или $K_{2}$ (см. п. 1.2)

3 а д а ч а 3. Дано $\Pi_{\text {III }}, \varphi_{\text {III }}, \rho, \Pi_{\max }^{\prime}$. Такая задача возникает в тех случаях, когда рабочий орган должен перемещаться на некотором участке с заданной постоянной скоростью или при заданном постоянном соотношении скоростей входного и выходного звеньев. В качестве примера подобной ситуации можно привести синтез закона движения листоразгоняющего устройства печатных машин, механизма раскладки нити текстильных машин, механизмов подачи инструмента станков-автоматов и др. На рис. 1.4, а приведена типовая схема листоразгоняющего механизма (форграйфера) листовой печатной машины. Рычаг 4, являющийся ведомым звеном кулачково-рычажного механизма (звенья 1-4), своими клапанами 6 схватывает лист 5 в состоянии покоя, разгоняет его до постоянной окружной скорости печатного цилиндра 7 и передаёт его в клапаны цилиндра.

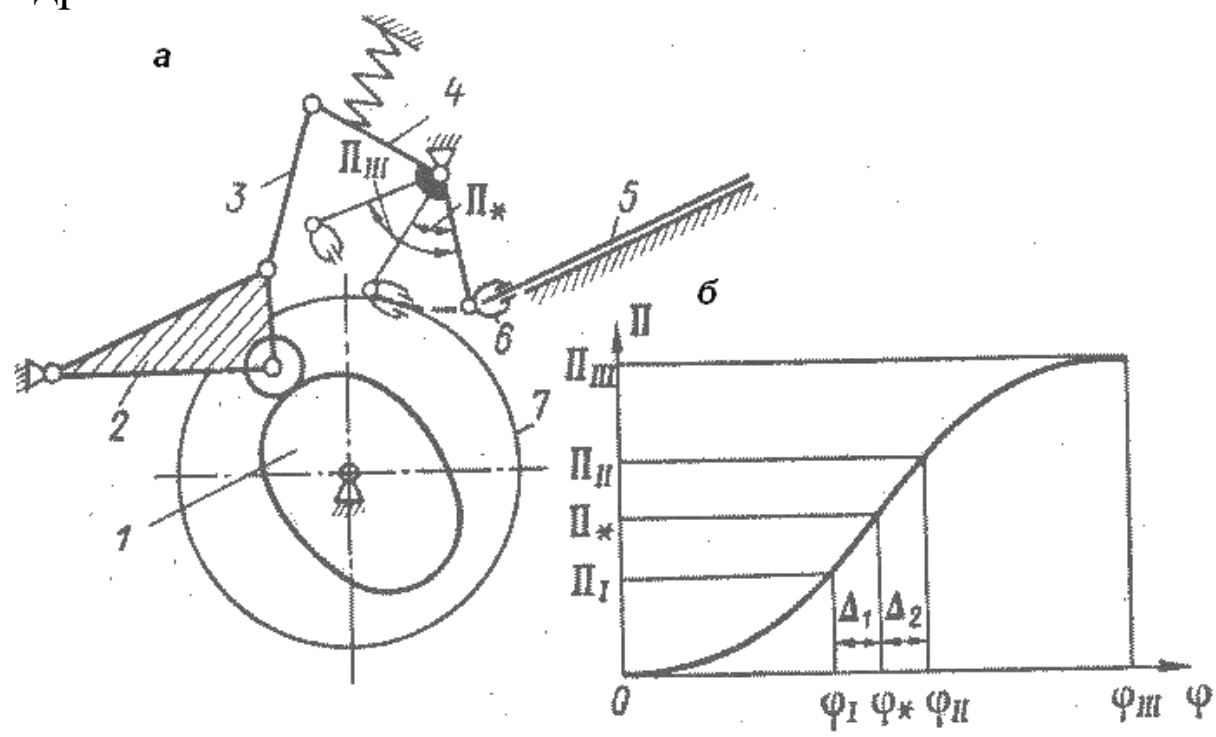

Рис. 1.4. Кинематическая схема и график функции положения листоразгоняющего механизма печатной машины (форграйфера) 
В данном механизме фиксируется значение $\Pi_{\max }^{\prime}=R_{2} / R_{1}$, где $R_{1}, R_{2}-$ радиусы цилиндра и рычага, и в функции положения появляется участок постоянной скорости (рис.1.4, б). Поскольку в этой задаче в отличие от предыдущих задано максимальное значение первой геометрической передаточной функции, приходится отказаться от задания относительной величины участка постоянной скорости, определяемой параметрами $\zeta_{n}$ или $\zeta_{\varphi}$. Из $(1.7),(1.8)$ следует очевидное соотношение

$$
\zeta_{n} / \zeta_{\varphi}=\Pi_{\max }^{\prime} / \bar{\Pi}^{\prime}>1
$$

где $\Pi^{\prime}=\Pi_{\text {III }} / \varphi_{\text {III }}-$ среднее значение первой геометрической передаточной функции на всем интервале движения $\left(\bar{\Pi}^{\prime}<\Pi_{\max }^{\prime}\right)$.

Решая систему уравнений (1.15) и (1.22) относительно $\zeta_{n}$, находим

$$
\zeta_{n}=\frac{\theta_{1 \max }^{\prime} U-\Pi_{\max }^{\prime} / \overline{\Pi^{\prime}}}{\theta_{1 \max }^{\prime} U-1} .
$$

После определения по формуле (1.19) параметра $\zeta_{n}$, исходные условия отвечают задаче 2. Условия существования решений определяются следующими очевидными требованиями: $0 \leq \zeta_{n} \leq 1$. . При $\zeta_{n}=0$ участок постоянной скорости на графике П(ф) (см. рис.1.4,б) стягивается в точку; при $\zeta_{n} \rightarrow 1$ исчезают участки разбега и выбега, что приводит к удару в начале и конце хода. Приведенные условия накладывают следующие ограничения на исходные данные:

$$
\Pi_{\mathrm{III}}<\varphi_{\mathrm{III}} \Pi_{\max }^{\prime} \leq \Pi_{\mathrm{III}} U \theta_{1 \text { max }}^{\prime} .
$$

Нередко исходные условия синтеза закона движения таковы, что наряду с заданным максимальным значением первой геометрической передаточной функции $\Pi_{\max }^{\prime}$ определенным образом фиксируется протяженность участка постоянной скорости. Это дополнительное требование может быть выполнено, если исключить из исходных данных ход рабочего органа П ствующий ему фазовый угол $\varphi_{\text {III }}$.

На основании формул (1.10), (1.19) в первом случае находим

$$
\Pi_{\mathrm{III}}=\frac{1+\zeta_{\varphi}\left(\theta_{1 \max }^{\prime} U-1\right)}{\theta_{1 \max }^{\prime} U} \Pi_{\max }^{\prime} \varphi_{\mathrm{III}}
$$

а во втором

$$
\varphi_{\mathrm{III}}=\frac{\Pi_{\mathrm{III}}}{\Pi_{\max }^{\prime}}\left[\theta_{1 \max }^{\prime} U-\zeta_{n}\left(\theta_{1 \max }^{\prime} U-1\right)\right] .
$$

Формулы (1.21) и (1.22) сводят рассматриваемые случаи к исходным условиям задачи 3 . При этом остаются в силе условия существования решений, определяемые неравенствами (1.20). 
На основании рассмотренных типовых задач синтеза законов программного движения могут быть решены и другие задачи, в которых ряд ранее зафиксированных параметров варьируется на заданном интервале [6].

В заключение подчеркнём, что для объективного сопоставления различных типов законов движения, определяемых функциями $\theta_{i}\left(\tau_{i}\right)$, следует выразить $\Pi_{\max }^{\prime},\left|\Pi_{\max }^{\prime \prime},\right|\left|\Pi^{\prime} \Pi^{\prime \prime}\right|_{\max }$ через независимые исходные условия задачи. Как уже отмечалось, при этом нельзя в общем случае судить об этих критериях по одноименным безразмерным константам $\theta_{\text {max }}^{\prime}, \theta_{\text {max }}^{\prime \prime},\left(\theta^{\prime} \theta^{\prime \prime}\right)_{\max }$, так как структурные параметры закона движения в числе прочих факторов согласно уравнениям (1.11) зависят от константы $\theta_{i \max }^{\prime}$. Из этих уравнений, в частности, следует, что непосредственно по одноименным безразмерным константам можно сопоставлять законы движения лишь при одинаковых значениях $\theta_{i \max }^{\prime}$ •

Кроме рассмотренного выше подхода, когда при синтезе мы оперируем одним или несколькими семействами законов движения, сопоставляя их по динамическим критериям, возможен и другой подход, при котором в каждом отдельном случае создается принципиально новый тип закона движения. Такой подход обычно оправдан при решении специальных задач синтеза уникального характера.

\section{3. Простейшие критерии динамического синтеза}

При нелинейной функции положения, свойственной так называемым цикловым механизмам - кулачковым, рычажным, шаговым и т.п., динамические условия работы оказываются более напряженными по сравнению с механизмами с линейной функцией положения. Даже в идеальном цикловом механизме в силу $\ddot{\varphi}_{n} \neq 0$ возникают инерционные нагрузки, причем нередко весьма значительные. Кроме того, имеет место более невыгодная силовая связь между ведущим и ведомым звеньями.

Если, например, на ведомом звене $n$ приложена сила $F$, которая на ведущем звене уравновешивается моментом $M$, то в силу равенства работ на возможных перемещениях

$$
M=\Pi_{n}^{\prime}\left(\varphi_{1}\right) F
$$

Очевидно, что при $\Pi_{n}^{\prime} \neq$ const даже постоянная сила $F$ приводит к возникновению на входном звене переменного момента, способного возбуждать вынужденные колебания привода.

Представляет интерес еще один частный случай. Пусть сила $F$ является силой инерции ведомого звена $n$. Тогда, принимая для определенности, что ведомое звено совершает поступательное движение, при $\dot{\varphi}_{1}=$ const имеем

$$
|F|=m \dot{\varphi}_{1}^{2}\left|\Pi_{n}^{\prime \prime}\right|
$$

После подстановки в (1.3) получаем 


$$
|M|=m \dot{\varphi}_{1}^{2}\left|\Pi_{n}^{\prime} \Pi_{n}^{\prime \prime}\right|
$$

Легко убедиться, что $\Pi_{n}^{\prime} \Pi_{n}^{\prime \prime}=\left(m \dot{\varphi}_{1}^{3}\right)^{-1} \frac{d T_{n}}{d t}$, где $T_{n}-$ кинетическая энергия звена $n ; d T_{n} / d t-$ кинетическая мощность.

Выражения (1.23) - (1.25) свидетельствуют о том, что геометрические характеристики существенно влияют на динамику механизма. Поэтому экстремальные значения функций $\left|\Pi^{\prime}\right|_{\max }$, $\left|\Pi^{\prime \prime}\right|_{\max }$, $\left|\Pi^{\prime} \Pi^{\prime \prime}\right|_{\max }$ могут быть использованы в качестве простейших динамических критериев, с помощью которых производится сопоставление различных законов движения, а также синтез новых законов, обладающих в определенном смысле оптимальными свойствами. Для контроля за пульсацией инерционных нагрузок на ведомом и ведущем звеньях могут быть использованы критерии

$$
K_{1}=\Pi_{\max }^{\prime \prime}+\xi_{1}\left|\Pi_{\min }^{\prime \prime}\right| ; K_{2}=\left(\Pi^{\prime} \Pi^{\prime \prime}\right)_{\max }+\xi_{2}\left|\left(\Pi^{\prime} \Pi^{\prime \prime}\right)_{\min }\right| .
$$

Здесь $\xi_{1}$ и $\xi_{2}-$ некоторые весовые коэффициенты, с помощью которых можно отразить степень важности положительной и отрицательной составляющих.

Вопросы, связанные с определением геометрических характеристик механизмов, освещены во многих монографиях и учебных пособиях [14 -17]. Здесь лишь подчеркнем, что по способу формирования геометрических характеристик механизмы можно разделить на две группы: механизмы дискретного синтеза и функционального синтеза. К первой группе относят механизмы типа рычажных, у которых при синтезе определению подлежит лишь конечное число параметров. Геометрические характеристики таких механизмов, по сути дела, заложены в их схеме, и поэтому рациональным выбором параметров можно лишь приблизиться к заданной функции положения. Ко второй группе относятся механизмы типа кулачковых, в которых профилированием рабочих поверхностей можно непосредственно реализовать заданную функцию положения. Это во многих случаях существенно расширяет возможности учета динамических факторов при синтезе подобных механизмов.

Приведенные критерии, основанные на геометрических представлениях, разумеется, носят ограниченный характер и не могут исчерпать динамическую задачу (см. ниже), тем не менее, их использование оказывается весьма полезным, особенно на начальном этапе решения таких задач. 


\section{ГЛАВА 2. СИНТЕЗ ЗАКОНОВ ДВИЖЕНИЯ РАБОЧЕГО ОРГАНА ПРИ УЧЕТЕ УПРУГОСТИ ЗВЕНЬЕВ ЦИКЛОВОГО МЕХАНИЗМА}

\section{1. Общее решение}

При конструировании высокоскоростных кулачковых механизмов необходимо провести не только кинематический анализ, когда все звенья механизма принимаются абсолютно жесткими, но и динамический анализ, при котором учитывается податливость звеньев. Одна из простейших динамических моделей, к которой может быть приведено большое число конструктивных модификаций механизмов с поступательным движением ведомого звена, представлена на рис. 2.1,а.

a

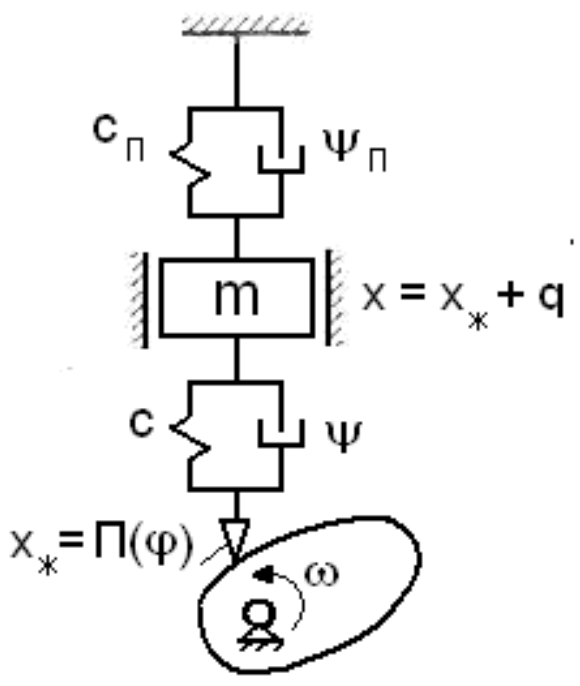

6

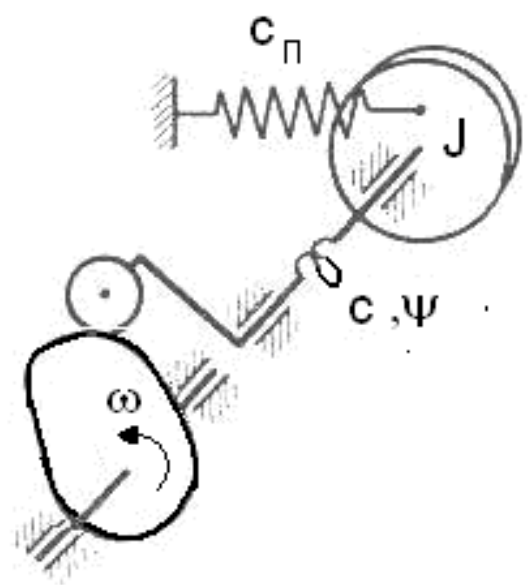

Рис. 2.1. Кулачковые механизмы с упругим толкателем

Запишем дифференциальное уравнение для данной модели при поступательном движении толкателя и $\quad \omega=$ const $\quad$ (рис. 2.1,a):

$$
m \ddot{q}+b \dot{q}+\left(c+c_{\mathrm{i}}\right) q=-F-m \ddot{x}_{*}-c_{\mathrm{i}} x_{*}-F_{0},
$$

где $q$ - деформация; $m, c$ - приведенные масса и коэффициент жесткости толкателя; $b$ - коэффициент эквивалентного линейного сопротивления $x_{*}=\Pi(\varphi)-$ программный закон движения; $F-$ внешняя сила; $c_{i \mathrm{i}}, F_{0}-$ коэффициент жесткости и предварительное усилие замыкающей пружины; $\varphi=\omega t$ - угол поворота входного звена.

Если выходное звено механизма является коромыслом, совершающим угловые перемещения вокруг неподвижной оси (рис. 2.1,б), вместо массы следует принять момент инерции $J$, а вместо жесткости в продольном направлении - крутильную жесткость. 
Поделив все члены уравнения (2.1) на $m$, запишем его в следующем виде:

$$
\ddot{q}+2 n \dot{q}+k^{2} q=W(t),
$$

где $2 n=b / m ; \quad k^{2}=\left(c+c_{\text {ï }}\right) / m$.

Правую часть уравнения (2.2), которую будем называть функцией возбуждения, можно записать так:

$$
W(t)=-\left(\Pi^{\prime \prime} \omega^{2}+k_{\mathrm{i}}^{2} \Pi+h\right) .
$$

Здесь $k_{\Pi}^{2}=c_{\text {ï }} / m ; \quad h=\left(F+F_{0}\right) / m ; \quad \Pi^{\prime \prime}=d^{2} \Pi / d \varphi^{2}-$ вторая геометрическая передаточная функция. При линейной функции положения $П$, реализуемой, например, в зубчато-реечных и зубчатых передачах, а также в кулачковых механизмах на участке постоянной скорости $[5,6,15]$. Первое слагаемое формулы (2.3) отсутствует, поскольку $\Pi^{\prime}=$ const и $\Pi^{\prime \prime}=0$.

Пусть на некотором отрезке времени функции $\Pi, W$ и их производные не имеют разрывов непрерывности. Тогда решение уравнения (2.2) имеет вид

$$
q=\mathrm{e}^{-n t}\left(C_{1} \cos k_{1} t+C_{2} \sin k_{1} t\right)+Y(t) .
$$

Здесь $k_{1}=\sqrt{k^{2}-n^{2}} ; \quad Y(t)$ - частное решение неоднородного уравнения; $C_{1}$, $C_{2}$ - постоянные интегрирования, которые определим, исходя из начальных условий при $t=0: q(0)=q_{0} ; \dot{q}(0)=\dot{q}_{0}$.

Параметр $k_{1}$ называют круговой частотой свободных колебаний или собственной частотой. Поскольку $k_{1}=k \sqrt{1-\delta^{2}}$, а коэффициент $\delta=n / k$ при отсутствии специальных демпфирующих устройств обычно не превосходит значения 0,1 , можно принять $k_{1} \approx k$. Таким образом, влиянием сил сопротивления на собственную частоту, как правило, можно пренебречь. Кроме того, $c_{\mathrm{i}} \square c$, поэтому $k \approx \sqrt{c / m}$.

После определения $C_{1}$ и $C_{2}$ получаем $q=\mathrm{e}^{-n t}\left(q_{0} \cos k t+\frac{\dot{q}_{0}+n q_{0}}{k} \sin k t\right)-\mathrm{e}^{-n t}\left[Y(0) \cos k t+\frac{\dot{Y}(0)+n Y(0)}{k} \sin k t\right]+$ $+Y(t)$

Первая группа слагаемых описывает свободные колебания, частота которых равна собственной частоте, а амплитуда зависит от начальных условий. Амплитуды свободных колебаний при линейной силе сопротивления образуют бесконечно убывающую геометрическую прогрессию, знаменатель которой $\mathrm{e}^{-n T}$ (где $T=2 \pi / k$ - период колебаний) называют декрементом колебаний, а величину $\lambda=n T-$ логарифмическим декрементом. Этот параметр равен абсолютному значению натурального логарифма от отношения двух соседних амплитуд, разделенных периодом.

Вторая группа определяет так называемые сопровождающие колебания, частота которых также равна собственной частоте $k$, однако в отличие 
от свободных колебаний амплитуда здесь не зависит от начальных условий, а определяется разрывами частного решения и его производной при $t=0$. Наконец, третье слагаемое соответствует вынужденным колебаниям. Вид частного решения определяется видом функции возбуждения (см. ниже). Интегральную форму частного решения иногда называют формулой Дюамеля:

$$
Y^{0}(t)=\frac{1}{k} \int_{0}^{t} W(u) \exp [-n(t-u)] \sin k(t-u) d u .
$$

При использовании (2.6) вторая группа слагаемых в (2.5) в явном виде отсутствует, так как при этом $Y^{0}(0)=0, \quad \dot{Y}^{0}(0)$. В этом случае сопровождающие колебания выявляются уже после процедуры интегрирования.

\section{2. Вынужденные колебания при гармоническом возбуждении}

Правая часть дифференциального уравнения (2.1) отражает двоякий характер возбуждения колебаний - кинематический и силовой. При кинематическом возбуждении какая-либо точка или сечение принудительным образом перемещается в соответствии с заданным законом программного движения; такой точкой в данном случае является входное сечение толкателя, контактирующее с профилем кулачка. При силовом возбуждении колебания возбуждаются переменной во времени вынуждающей силой $F(t)$.

Сначала рассмотрим случай, когда $F=$ const, а закон движения описывается гармонической функцией $\Pi=0,5 x_{\max }(1-\cos \varphi)$. Тогда функция возбуждения (2.3) приводит

$$
W=W_{0}+W_{1} \cos \omega t,
$$

где $W_{0}=-\left[0,5\left(\omega^{2}+k_{\mathrm{i}}^{2}\right) x_{\max }+h\right] ; \quad W_{1}=-0,5 x_{\max }\left(\omega^{2}-k_{\mathrm{i}}^{2}\right)$.

При отсутствии разрывов функций П и $W$, как было показано выше, для установившегося режима $(t \rightarrow \infty) q \rightarrow Y$.

Частное решение $Y$ неоднородного дифференциального уравнения (2.2) ищем как сумму постоянной составляющей и гармонической функции:

$$
Y=A_{0}+A_{C} \cos \omega t+A_{S} \sin \omega t=A_{0}+A \cos (\omega t-\gamma),
$$

где $A_{0}=W_{0} / k^{2}$;

$$
\begin{gathered}
A=\sqrt{A_{C}^{2}+A_{s}^{2}}=0,5 x_{\max } \kappa_{k}(z) ; \\
\gamma=\operatorname{arctg}\left[2 \delta \mathrm{z} /\left(1-\mathrm{z}^{2}\right)\right] \quad(\gamma \in[0, \pi]) .
\end{gathered}
$$

Здесь $z=\omega / k-$ коэффициент частотной расстройки; $\delta=n / k ; \kappa_{k}(z)-$ коэффициент динамичности при кинематическом возбуждении, определяемый следующим образом:

$$
\kappa_{k}=\frac{\left|z^{2}-z_{i}^{2}\right|}{\sqrt{\left(1-z^{2}\right)^{2}+4 z^{2} \delta^{2}}} .
$$


Здесь $\quad z_{i}^{2}=k_{i}^{2} / k^{2}$.

Коэффициент динамичности $\kappa_{k}$ является безразмерной амплитудночастотной характеристикой (АЧХ). Согласно (2.9) он равен отношению амплитуды колебаний $A$ к амплитуде кинематического возбуждения $0,5 x_{\max }$. Характерный график АЧХ приведен на рис. 2.2, a. При $z \in\left[0, z_{1}\right]$ функция $\kappa_{k}(z)$ изменяется от значения $z_{i}^{2}$ до нуля, после чего возрастает до своего максимального значения $\kappa_{k \max }$. Используя условие экстремума $d \kappa_{k} / d z=0$, нетрудно убедиться, что значению $\kappa_{k \max }$ соответствует

$$
z_{*}=\sqrt{\frac{1-z_{i}^{2}\left(1-2 \delta^{2}\right)}{1-z_{i}^{2}-2 \delta^{2}}} .
$$

a

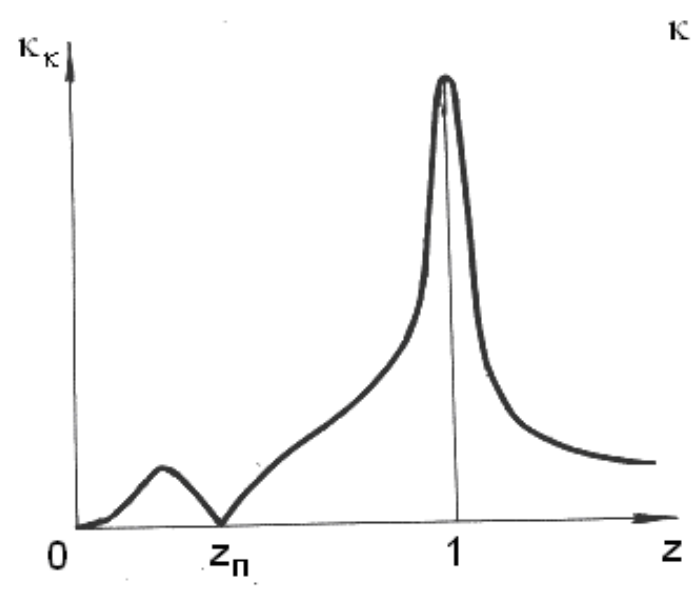

б

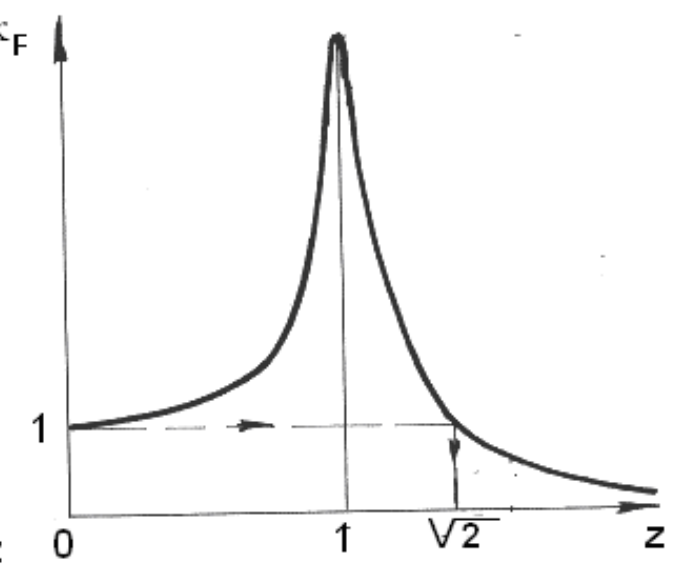

Рис. 2.2. Коэффициенты динамичности при кинематическом и силовом возбуждении

Обычно $z_{\Pi}^{2}<<1$ и $\delta<<1$, поэтому $z_{*} \approx 1$ и $\kappa_{k \max } \approx 1 / 2 \delta$. Значение $z=1(\omega=k)$ отвечает резонансу. В зарезонансной зоне $(z>1)$ коэффициент динамичности убывает, асимптотически приближаясь к значению $\kappa_{k}=1$. резонансу. В зарезонансной зоне $(z>1)$ коэффициент динамичности убывает, асимптотически приближаясь к значению резонансу $\left(\kappa_{k}=1\right)$. В зарезонансной зоне $(z>1)$ коэффициент динамичности убывает, асимптотически приближаясь к значению $\kappa_{k}=1$.

Большой интерес представляет режим $z=z_{\text {İ }}$, при котором $\omega=k_{\text {i }}$. При этом режиме $W_{1}=0$, а следовательно, $q \rightarrow A_{0}=$ const. Легко убедиться, что при разрыве кинематической связи между выходным и входным звеньями

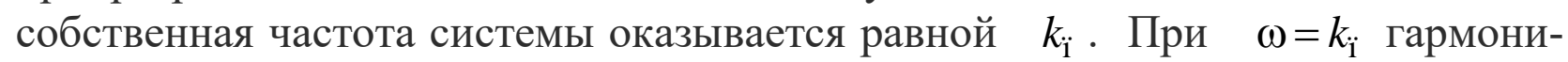
ческая составляющая восстанавливающей силы в замыкающей пружине и сила инерции переносного движения равны по величине и противоположны по направлению. 
Аналогичным способом при воздействии гармонической вынуждающей силы $F=F_{1} \cos (\omega t+\alpha)$ получаем $q=B \cos (\omega t+\alpha-\gamma)$, причем

$$
B=B_{\text {กิ் }} \kappa_{F} \text {, }
$$

где $B_{\text {กัò }}=F_{1} / c ; \quad \kappa_{F}=1 / \sqrt{\left(1-z^{2}\right)^{2}+4 z^{2} \delta^{2}}$. Здесь $B_{\text {ก̃ò }}-$ так называемая $\mathrm{cma}$ тическая амплитуда, равная деформации системы под действием амплитудного значения вынуждающей силы, приложенной в статических условиях; $\kappa_{F}$ - коэффициент динамичности при силовом возбуждении (рис. 2.2, б).

Из зависимостей $(2.8,2.9)$ следует, что вынужденные колебания по фазе смещены относительно функции возбуждения на величину $\gamma$. Зависимости $\gamma(\omega)$ или $\gamma(z)$ называют фазочастотной характеристикой (ФЧХ). На рис. 2.3 приведено семейство кривых $\gamma(z)$, отвечающее зависимости (2.9). При z $<<1$ имеем $\gamma \approx 0$, при $z=1$ (резонанс) $\gamma=\pi / 2$, при $z \rightarrow \infty$ колебания происходят в противофазе $(\gamma \rightarrow \pi)$.

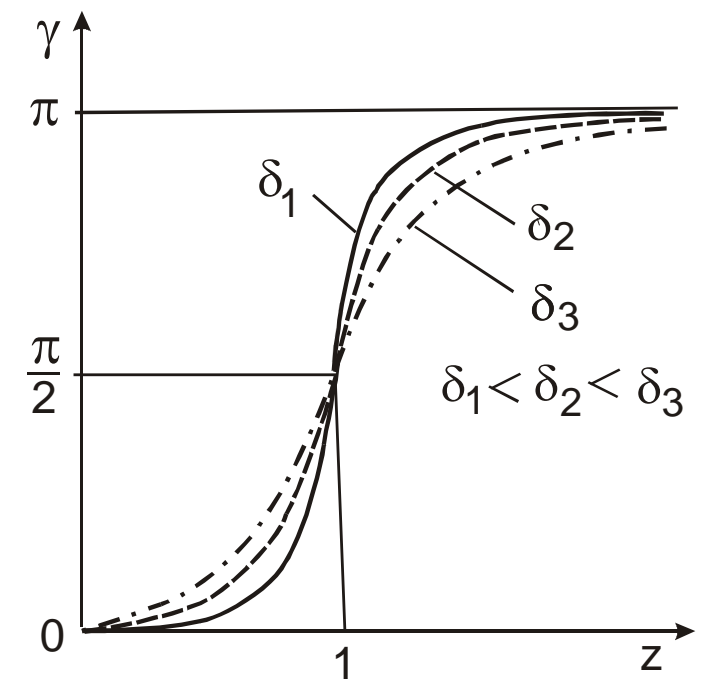

Рис.2.3. Фазочастотная характеристика

\section{3. Вынужденные колебания при периодической функции возбуждения}

Метод гармонического анализа. Выше мы анализировали вынужденные колебания при гармонической вынуждающей силе, являющейся частным случаем периодической вынуждающей силы. При периодическом силовом возбуждении (рис.2.4)) дифференциальное уравнение имеет вид

$$
a \ddot{q}+b \dot{q}+c q=F(t),
$$

где $F(t)=F(t+\tau)-$ периодическая вынуждающая сила; $\tau=2 \pi / \omega-$ период 


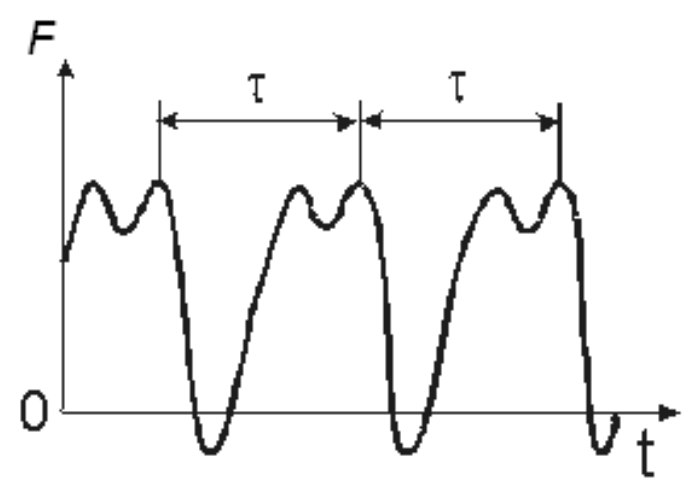

Рис. 2.4. Периодическое изменение вынуждающей силы

Представим функцию $F(t)$ в виде ряда Фурье:

$$
F(t)=F_{0}+\sum_{j=1}^{\infty}\left(F_{j c} \cos j \omega t+F_{j s} \sin j \omega t\right),
$$

где $\omega=2 \pi / \tau ; F_{0}, F_{j c}, F_{j s}-$ коэффициенты Фурье, определяемые следующим образом:

$$
\left.\begin{array}{l}
F_{0}=\tau^{-1} \int_{0}^{\tau} F(t) d t ; \\
F_{j c}=2 \tau^{-1} \int_{0}^{\tau} F(t) \cos j \omega t d t ; \\
F_{j s}=2 \tau^{-1} \int_{0}^{\tau} F(t) \sin j \omega t d t .
\end{array}\right\}
$$

Для многих типовых случаев $F_{0}, F_{j c}, F_{j s}$ приводятся в справочной литературе; кроме того, имеются стандартные программы, позволяющие находить коэффициенты Фурье, задаваясь определенным числом частных значений функции $F(t)$.

При построении решения уравнения (2.13) воспользуемся принщипом суперпозищии, справедливым для линейных систем. Применительно к данной задаче он состоит в том, что колебания, возникающие от суммы сил, можно определять как сумму колебаний от каждой силь в отдельности. Так как колебания при гармонической вынуждающей силе были рассмотрены выше, задачу можно считать принципиально решенной.

Представим (2.14) в следующем виде:

$$
F(t)=F_{0}+\sum_{j=1}^{\infty} F_{j} \sin \left(j \omega t+\alpha_{j}\right),
$$

где $F_{j}=\sqrt{F_{j c}^{2}+F_{j s}^{2}} ; \cos \alpha_{j}=F_{j s} / F_{j} ; \sin \alpha_{j}=F_{j c} / F_{j}$.

Каждый из членов ряда (2.16) называют гармоникой $j$. Деформация от постоянной составляющей $F_{0}$ равна $A_{0}=F_{0} / c$. Колебания от гармоники $j$ вынуждающей силы определяются на основании зависимостей (2.9), (2.10) 


$$
q_{j}=A_{j} \sin \left(j \omega t+\alpha_{j}-\gamma_{j}\right),
$$

где $A_{j}=A_{j \text { п̃ оั }} \kappa_{j} ; \quad \kappa_{j}=1 / \sqrt{\left(1-j^{2} z^{2}\right)^{2}+4 j^{2} z^{2} \delta^{2}} ; \quad z=\omega / k ; \quad A_{j \text { п̃ò }}=F_{j} / c-$ статическая амплитуда гармоники $j ; \quad k=\sqrt{c / a} ; \quad \gamma_{j}=\operatorname{arctg}\left[2 \delta j z /\left(1-j^{2} z^{2}\right)\right]$ - фазочастотная характеристика.

(При кинематическом возбуждении следует воспользоваться зависимостями (2.9), (2.10) при замене $z$ на $j z)$

Итак, окончательно имеем

$$
q=F_{0} / c+\sum_{j=1}^{\infty} q_{j}
$$

При $j z=1 \quad(j \omega=k)$ возникает резонанс гармоники $j$; при этом $\kappa_{j}$ ðåç $=1 /(2 \delta)$.

Решение (2.18) является математически точным, однако поскольку при суммировании членом ряда Фурье приходится ограничиваться конечным числом гармоник $j_{\max }$, с инженерных позиций оно оказывается приближенным. При выборе $j_{\max }$ можно руководствоваться следующими соображениями.

Во-первых, должны быть учтены наиболее значительные члены разложения $F_{j}$. Во-вторых, чтобы не отсечь резонансный режим, следует потребовать $j_{\max }>k / \omega+(1 \div 2)$.

Отметим, что стремление сохранить в ряде Фурье большое число гармоник нередко создаёт лишь иллюзию повышения точности, поскольку точность определения высших гармоник обычно не велика. Поэтому рассматриваемый способ расчета вынужденных колебаний целесообразно использовать в задачах динамики механизмов при отсутствии ударных нагрузок, а также при непрерывных и дифференцируемых функциях положения, обладающих повышенной «гладкостью». Это свойственно, например, простым рычажным механизмам и эксцентрикам, когда при расчетах можно ограничиваться малым числом учитываемых гармоник.

Замкнутая форма решения. При установившихся режимах $(t \rightarrow \infty)$, согласно (2.5), свободные колебания за счет экспоненциального множителя быстро затухают. На первый взгляд аналогичный вывод можно сделать и относительно сопровождающих колебаний. Однако последнее справедливо лишь при условии, что функции П и $W$ и ее производные не терпят разрывов непрерывности. В противном случае сопровождающие колебания возбуждаются не только при $t=0$, но и в моменты времени, соответствующие этим разрывам.

Обобщенная координата $q$, характеризующая колебательный процесс, в рассматриваемом примере непосредственно описывает динамическую ошибку выходного звена, поскольку абсолютная координата выходного звена определяется как $y=x+q$; соответственно имеем динамические ошибки по скорости $\dot{q}$ и ускорению $\ddot{q}$. 
Динамическая ошибка $q$ в данном случае одновременно характеризует виброактивность механизма, определяемую реактивным моментом $M \approx c q \Pi^{\prime}$, который передается на входное звено (здесь и ниже штрих отвечает производной по углу поворота входного звена $\varphi$ ). ошибок.

Ниже рассмотрим ряд типовых случаев определения динамических

Разделим ось времени на участки, в пределах которых функции $x=\Pi(\varphi)$ и $W(t)$ непрерывны и дифференцируемы. Тогда для каждого участка справедливы зависимости вида (2.4). Так, например, для участка $i+1$

$$
q_{i}=\mathrm{e}^{-n\left(t-t_{i}\right)}\left[C_{1 i} \cos k\left(t-t_{i}\right)+C_{2 i} \sin k\left(t-t_{i}\right)\right]+Y_{i+1}(t) \quad\left(t>t_{i}\right) .
$$

Для определения констант $C_{1 i}$ и $C_{2 i}$ воспользуемся тем обстоятельством, что абсолютная координата $y=x+q$ и ее производная $\dot{y}=\dot{x}+\dot{q}$ на границе участков сохраняют непрерывность. Тогда

$$
x_{i}^{-}+q_{i}^{-}=x_{i}^{+}+q_{i}^{+} ; \quad \dot{x}_{i}^{-}+\dot{q}_{i}^{-}=\dot{x}_{i}^{+}+\dot{q}_{\dot{i}}^{+} .
$$

Здесь индекс «+» соответствует моменту времени $t_{i}+\varepsilon(\varepsilon-$ бесконечно малая величина), а индекс «-»- $t_{i}-\varepsilon$. Таким образом, условия стыкования решения на границах участков могут быть записаны следующим образом:

$$
q_{i}^{+}=q_{i}^{-}-\Delta x_{i} ; \quad \dot{q}_{1}^{+}=\dot{q}_{i}^{-}-\Delta \dot{x}_{i},
$$

где $\Delta x_{i}=x_{i}^{+}-x_{i}^{-} ; \quad \Delta \dot{x}_{i}=\dot{x}_{i}^{+}-\dot{x}_{i}^{-}=\omega\left(\Pi_{i}^{\prime+}-\Pi^{\prime}{ }_{i}^{-}\right)$.

Для выявления динамических эффектов, сопутствующих этим разрывам, воспользуемся формой решения в виде ряда по производным функции возмущения $W$, которая представляет особый интерес в задачах синтеза законов движения кулачковых механизмов. Если частное решение $Y$ записать в интегральной форме (2.6) и подвергнуть его последовательному интегрированию по частям, то получаем $[6,7,10]$

где

$$
Y^{*} \approx \sum_{m=1,3,5 \ldots}^{\infty}(-1)^{0,5(m-1)} w_{m(t)}
$$

Здесь $Y^{*}$ - новая форма частного решения, из которого уже полностью исключены сопровождающие колебания (в дальнейшем звездочка опускается). Используя (2.20) и (2.21), выразим константы $C_{1 i}$ и $C_{2 i}$ : 


$$
\begin{aligned}
& C_{1 i}=-\left[\Delta x_{i}+\sum_{m=1,3 \ldots}^{\infty}(-1)^{0,5(m-1)} \Delta w_{i m}\right] ; \\
& C_{2 i}=-\left[\frac{\Delta \dot{x}_{i}}{k}+\sum_{m=2,4 \ldots}^{\infty}(-1)^{0,5 m-1} \Delta w_{i m}\right],
\end{aligned}
$$

где $\Delta w_{i m}=w_{m}\left(t_{i}+0\right)-w_{m}\left(t_{i}-0\right)$.

На основании (2.22), имеем

$$
q_{i}=D_{i} \exp \left[-n\left(t-t_{i}\right)\right] \sin \left[k\left(t-t_{i}\right)+\alpha_{i}\right]
$$

где $D_{i}=\sqrt{C_{1 i}^{2}+C_{2 i}^{2}} ; \sin \alpha_{i}=C_{1 i} / D_{i} ; \cos \alpha_{0}=C_{2 i} / D_{i}$.

В дальнейшем для краткости амплитуду $D_{i}$ будем называть скачком.

При $\omega<k$ ряды по $m$ (2.21), (2.23) обычно быстро сходятся. В частном случае, если функция $W(t)$ представлена в виде полинома, эти ряды имеют конечное число членов. В приведенных зависимостях сохраняется большая наглядность при выявлении источников возбуждения сопровождающих колебаний, что позволяет на стадии динамического синтеза активно «вмешиваться» в процесс снижения виброактивности.

Помимо описанного аналитического метода построения решения (2.24) можно также осуществить интегрирование исходного дифференциального уравнения (2.2) численными методами (например, методом Рунге - Кутта) при обязательном соблюдении условий (2.20).

Анализируя формулу (2.19), легко заметить, что с одной стороны, система периодически возбуждается за счет внешних возмущений, а с другой существует постоянный отток энергии за счет диссипативных сил, снижающих уровень колебаний. При $\omega=$ const в результате воздействия этих двух факторов (строго говоря, при $t \rightarrow \infty$, а практически достаточно быстро) возникает некоторый установившийся колебательный режим, который нам предстоит описать.

Наиболее естественным способом определения динамических ошибок в этом случае, на первый взгляд, является интегрирование исходного дифференциального уравнения до выхода на установившийся режим. Однако такой путь при использовании численных методов обычно приводит к значительной накопленной погрешности и повышенной трудоемкости расчета. Поэтому воспользуемся более точным и экономичным методом построения замкнутой формы решения.

Исследуем поведение колебательной системы на произвольно выбранном периоде $\tau=2 \pi / \omega$. Принимая за нуль отсчета момент начала данного периода, представим решение (2.22) как

$$
q=q^{0}\left(C_{1}, C_{2}, t\right)+Y^{0}(t)
$$


где $q^{0}=\mathrm{e}^{-n t}\left(C_{1} \cos k t+C_{2} \sin k t\right) ; \quad Y^{0}(t)-$ частное решение при нулевых начальных условиях.

Подчеркнем, что в данном случае нам неизвестны начальные условия, так как рассматриваемому периоду при установившемся режиме предшествует бесчисленное количество периодов. Численно интегрируя исходное дифференциальное уравнение (2.2) при нулевых начальных условиях, получаем на основании (2.5) $Y(t)=Y^{0}(t)$ (здесь, так же как и при использовании формулы Дюамеля $Y^{0}(0)=0 ; \dot{Y}^{0}(0)=0 ;$ при этом $\left.q^{0}(0,0, t)=0\right)$.

Абсолютное движение толкателя складывается из переносного движения, соответствующего в нашем случае заданному программному движению идеального механизма, и относительного движения за счёт возбуждаемых колебаний: $x(t)=x_{*}(t)+q(t)$. При переходе к следующему циклу в силу условий периодичности $y(0)=x(\tau)+q(\tau)$ и $\dot{y}(0)=\dot{x}(\tau)+\dot{q}(\tau)$, поэтому на основании зависимостей (2.23) и (2.25) имеем

$$
\begin{aligned}
& q^{0}\left(C_{1}, C_{2}, 0\right)=q^{0}\left(C_{1}, C_{2}, \tau\right)+Y^{0}(\tau)-\Delta x_{0} ; \\
& \dot{q}^{0}\left(C_{1}, C_{2}, 0\right)=\dot{q}^{0}\left(C_{1}, C_{2}, \tau\right)+\dot{Y}^{0}(\tau)-\Delta \dot{x}_{0},
\end{aligned}
$$

где $\Delta x_{0}=x(\tau)-x(0), \Delta \dot{x}=\dot{x}(\tau)-\dot{x}(0)$.

Поскольку значения $Y^{0}(\tau)$ и $\dot{Y}^{0}(\tau)$ нам уже известны, остается решить полученную систему алгебраических уравнений относительно двух неизвестных $C_{1}, C_{2}$.

Для более наглядной иллюстрации этой процедуры сначала примем $n=0$. Тогда система (2.26) принимает вид

$$
\begin{aligned}
& C_{1}=C_{1} \cos k t+C_{2} \sin k t+Y^{0}(\tau)-\Delta x_{0} ; \\
& k C_{2}=-C_{1} \sin k t+C_{2} k \cos k t+\dot{Y}^{0}(\tau)-\Delta \dot{x}_{0} .
\end{aligned}
$$

Решение этой системы алгебраических уравнений приводится к виду

$$
\begin{aligned}
& C_{1}=\frac{Y^{0}(\tau)-\Delta x_{0}}{2}+\frac{\dot{Y}^{0}(\tau)-\Delta \dot{x}_{0}}{2 k} \operatorname{ctg} \frac{\mathrm{k} \tau}{2} ; \\
& C_{2}=\frac{\dot{Y}^{0}(\tau)-\Delta \dot{x}_{0}}{2 k}+\frac{Y^{0}(\tau)-\Delta x_{0}}{2} \operatorname{ctg} \frac{\mathrm{k} \tau}{2} .
\end{aligned}
$$

При $k \tau / 2=j \pi(j=1,2, \ldots)$, что отвечает $j \omega=k$ (резонанс гармоники $\omega)$, имеем $C_{1} \rightarrow \infty, C_{2} \rightarrow \infty$. Естественно, такой же результат мы получили и при использовании метода гармонического анализа (см. п.2.2).

При $n \neq 0$ после аналогичных, хотя и более громоздких, выкладок и некоторых упрощений получаем 


$$
\begin{aligned}
& C_{1}=\frac{\left[Y^{0}(\tau)-\Delta x_{0}\right]\left(1-\mathrm{e}^{-\lambda N} \cos 2 \pi N\right)+\left[\dot{Y}^{0}(\tau)-\Delta \dot{x}_{0}\right] k^{-1} \mathrm{e}^{-\lambda N} \sin 2 \pi N}{1-2 \mathrm{e}^{-\lambda N} \cos 2 \pi N+\mathrm{e}^{-2 \lambda N}} ; \\
& C_{2}=\frac{\left.\left[\dot{Y}^{0}(\tau)-\Delta \dot{x}_{0}\right] k^{-1}\left(1-\mathrm{e}^{-\lambda N} \cos 2 \pi N\right)-\left[Y^{0}(\tau)-\Delta x_{0}\right] \mathrm{e}^{-\lambda N} \sin 2 \pi N\right)}{1-2 \mathrm{e}^{-\lambda N} \cos 2 \pi N+\mathrm{e}^{-2 \lambda N}},
\end{aligned}
$$

где $N=k / \omega ; \lambda-$ логарифмический декремент.

Тем самым найдены начальные условия, соответствующие установившемуся колебательному режиму:

$$
q_{0}=C_{1} ; \quad \dot{q}_{0}=C_{2} k-C_{1} n=k\left(C_{2}-C_{1} \delta\right) \approx k C_{2} \quad(\delta=n / k \square 1) .
$$

Повторив процедуру интегрирования с этими начальными условиями, находим окончательное решение $q(t)$, определяющее динамическую ошибку.

В соответствии с (2.29) амплитуда сопровождающих колебаний в начале цикла равна

$$
D_{0}=\sqrt{C_{1}^{2}+C_{2}^{2}}=\mu \sqrt{\left[Y^{0}(\tau)-\Delta x_{0}\right]^{2}+k^{-2}\left[\dot{Y}^{0}(\tau)-\Delta \dot{x}_{0}\right]}
$$

где $\mu-$ коэффициент накопления возмущений, определяемый как

$$
\mu=\frac{1}{\sqrt{1-2 \mathrm{e}^{-\lambda N} \cos 2 \pi N+\mathrm{e}^{-2 \lambda N}}} .
$$

Семейство кривых $\mu(N, \lambda)$, где $N=k / \omega$, приведено на рис. 2.5. Коэффициент $\mu$ может быть как больше единицы (усиление колебаний), так и меньше (ослабление колебаний). Максимальное значение $\mu^{+}$лежит в окрестности целых $N$, а минимальное значение $\mu^{-},-$когда $2 N$ - нечетное число. Из формулы (2.32) следует

$$
\mu^{+}=[1-\exp (-\lambda N)]^{-1} ; \quad \mu^{-}=[1+\exp (-\lambda N)]^{-1} .
$$

Значению $\mu^{+}$отвечает совпадение фаз ранее возбужденных колебаний и колебаний в рассматриваемом цикле; при $\mu=\mu^{-}$эти колебания находятся в противофазе. 


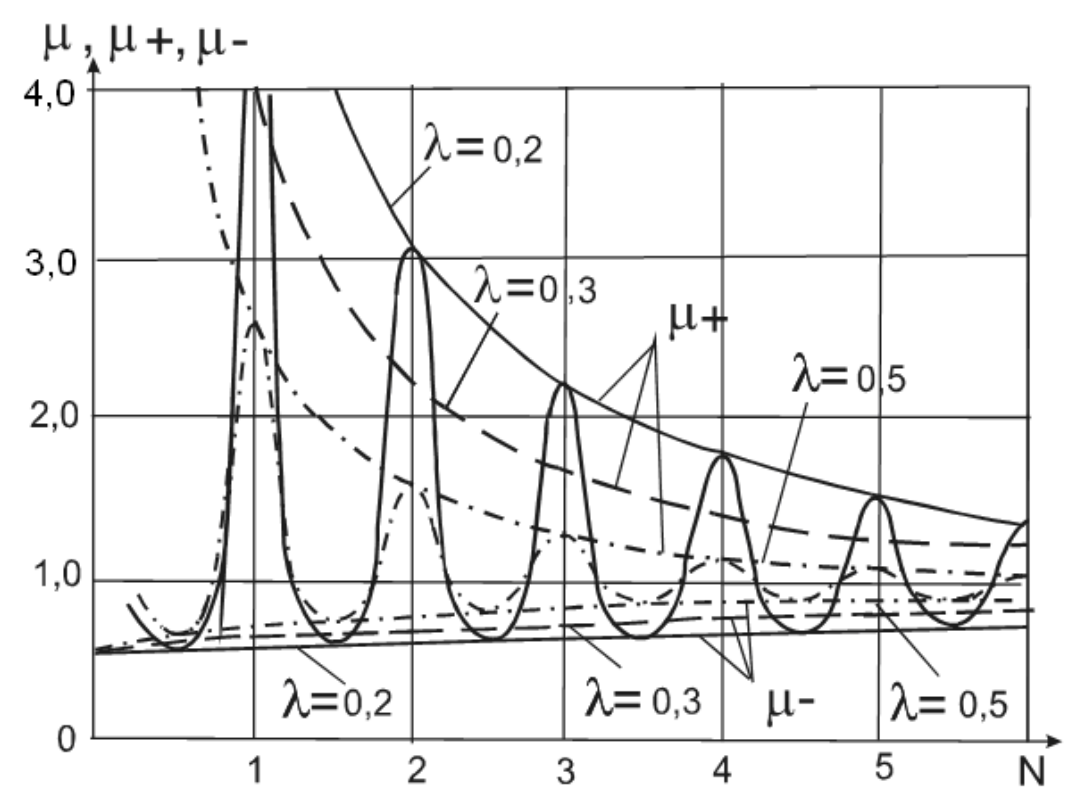

Рис. 2.5. Графики коэффициентов накопления возмущений

Подчеркнем важную особенность этого способа, связанную с возможностью рационального сочетания численных и аналитических методов: численным интегрированием с использованием компьютерных программ здесь определено лишь частное решение на ограниченном отрезке времени; условия же, отвечающие выходу на установившийся режим, выявлены аналитически. Последнее существенно сказывается на точности решения. Применение численно-аналитического способа особенно целесообразно в тех случаях, когда в геометрических передаточных функциях или внешних силах имеются разрывы непрерывности, что обычно характерно для кулачковых механизмов.

Разумеется, при относительно простых функциях возбуждения получение замкнутой формы решения можно осуществить и без обращения к численным методам, непосредственно используя решение (2.5) при учете (2.28) и аналитическую форму частного решения.

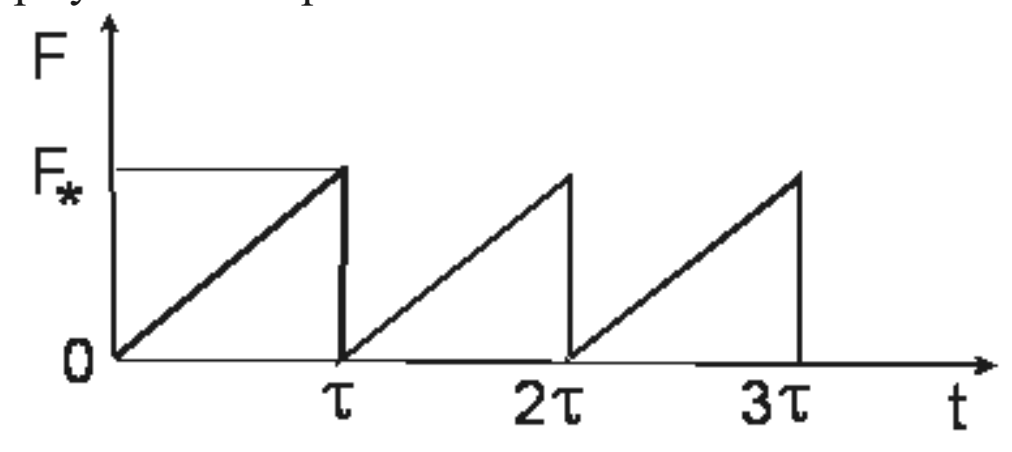

Рис.2.6. График $F(t)$

Пусть, например, периодическая вынуждающая сила имеет вид пилообразной характеристики (рис.2.6). При $0<t<\tau$ частное решение при $n=0$ имеет вид $Y=B t$,где $B=F_{*} /(c \tau)$. Тогда $Y(0)=0 ; Y(\tau)=B \tau ; \dot{Y}(0)=\dot{Y}(\tau)=B$; $\Delta Y=B \tau ; \quad \Delta \dot{Y}=0$, и согласно (2.28) $C_{1}=0,5 B \tau ; \quad C_{2}=-0,5 B \tau \operatorname{ctg}(k \tau / 2)$. 
В приведенном выше примере частное решение было найдено аналитическим способом. При более сложной форме описания вынуждающей силы целесообразно воспользоваться численно-аналитическим способом, который состоит из следующих этапов:

- численное интегрирование исходного дифференциального уравнения при нулевых начальных условиях (например, методом Рунге-Кутта); при этом находим

$$
\Delta Y=Y(\tau) \text { и } \Delta \dot{Y}=\dot{Y}(\tau) ;
$$

- определение констант $C_{1}$ и $C_{2}$ и начальных условий, отвечающих установившемуся режиму, по формулам (2.28), (2.29);

- повторное численное интегрирование с полученными начальными условиями.

При этом способе численным интегрированием найдены лишь отдельные промежуточные функции, вычисленные на ограниченном отрезке времени, который равен одному периоду $\tau$, в то время как установившийся режим $(t \rightarrow \infty)$ определяется аналитически с помощью условий периодичности. Последнее, как уже отмечалось, существенно уменьшает накопленную погрешность, что сказывается на повышении точности решения и, кроме того, снижает трудоемкость расчета. 


\section{ГЛАВА З. ВИБРОАКТИВНОСТЬ И ДИНАМИЧЕСКИЕ ОШИБКИ}

\section{1. Оптимизация с помощью коррекции геометрических характеристик закона движения}

Предварительные замечания. Виброактивность механизма определяется колебательной нагрузкой, передаваемой со стороны механизма на привод машины и через опоры на ее корпус. Снижение виброактивности механических систем и динамических ошибок является одной из центральных проблем динамического синтеза, решение которой во многом определяет производительность и техническое совершенство машинного оборудования. Исследование этой проблемы развивается в нескольких направлениях. Одно из первых по времени возникновения направлений связано с рациональным синтезом законов движения, обладающих оптимальными свойствами, исходя из выбранного динамического критерия.

Сначала динамические критерии полностью базировались на кинетостатической модели, не учитывающей упругость звеньев и связанные с ней колебательные процессы. При этом динамическая задача по существу полностью сводилась к геометрической. По мере роста рабочих скоростей машин стала проявляться ограниченность такого подхода. Действительно, как следует из уравнений (2.1), (2.2), характеристики законов движения входят в правую часть уравнений, и ими во многом определяется функция возбуждения $W(t)$, а следовательно, и возбуждаемые колебания. Уменьшение функции возбуждения может быть также достигнуто за счет установки специальных разгружающих устройств.

Другое направление динамического синтеза связано со снижением динамических ошибок за счет целенаправленного изменения параметров колебательной системы.

Следует иметь в виду, что решение той или иной частной задачи снижения виброактивности нередко носит локальный характер и иногда связано с повышением вибраций в другой части системы. Так, например, уравновешивание механизма с помощью противовесов приводит к увеличению переменной части приведенного момента инерции со всеми вытекающими отсюда отрицательными последствиями. Поэтому для инженера очень важен комплексный подход к проблеме, позволяющий в каждом отдельном случае находить компромиссное решение.

Источники возбуждения сопровождающих колебаний. Сначала определим сопровождающие колебания, связанные с разрывом непрерывности функций $x=\Pi(\varphi), \dot{x}=\Pi^{\prime}(\varphi) \omega, W$ в момент времени $t=t_{i}$ на границе двух участков $i$ и $i+1$. Как было установлено выше, колебания на участке i+1 при этом описываются зависимостью (2.19). Максимальное значение дополнительных ускорений, вызванных скачком $D_{i}$, равно 


$$
\left|\Delta \ddot{q}_{i}\right|_{\max }=k^{2} D_{i} .
$$

Ниже приведены пояснения к рассмотренным в табл. 3.1 типовым случаям.

\section{Таблица 3.1}

Расчетные зависимости для учета разрывов непрерывности геометрических характеристик механизма

\begin{tabular}{c|l|c|c|c}
\hline Тип скачка & $D$ & $|\Delta \ddot{q}|_{\max }$ & $\alpha_{1}$ & $\alpha_{2}$ \\
\hline$\Delta \Pi \neq 0$ & $|\Delta \Pi|$ & $k^{2}|\Delta \Pi|$ & $3 \pi / 2$ & $\pi / 2$ \\
\hline$\Delta \Pi^{\prime} \neq 0$ & $\omega_{0}\left|\Delta \Pi^{\prime}\right| / k$ & $k \omega_{0}\left|\Delta \Pi^{\prime}\right|$ & $\pi$ & 0 \\
\hline$\Delta \Pi^{\prime \prime} \neq 0$ & $\omega_{0}^{2}\left|\Delta \Pi^{\prime \prime}\right| / k^{2}$ & $\omega_{0}^{2}\left|\Delta \Pi^{\prime \prime}\right|$ & $\pi / 2$ & $3 \pi / 2$ \\
\hline$\Delta \Pi^{\prime \prime \prime} \neq 0$ & $\omega_{0}^{2}\left|\Delta \Pi^{\prime \prime \prime}\right| / k^{3}$ & $\omega_{0}^{3}\left|\Delta \Pi^{\prime \prime \prime}\right| / k$ & 0 & $\pi$ \\
\hline
\end{tabular}

Примечание: если рассматриваемое значение $\Delta \Pi, \Delta \Pi^{\prime}, \Delta \Pi^{\prime \prime}$ или $\Delta \Pi^{\prime \prime \prime}$ положительно, то $\alpha=\alpha_{1}$; в противном случае $\alpha=\alpha_{2}$.

Влияние разрывов непрерывности геометрических характеристик на значения $D, \alpha,|\Delta \ddot{q}|_{\max }$ определяются зависимостями (2.21) - (2.24). Для цикловых механизмов наиболее существенные скачки конкретизированы в табл.3.1. (Здесь и ниже индекс $i$ опущен). Каждая строка таблицы соответствует нарушению непрерывности лишь одной характеристики; при этом остальные функции предполагаются непрерывными.

Разрыв непрерывности функции положения $\triangle$ П. Этот случай в чистом виде не может быть реализован, однако к нему близок случай ступенчатого изменения профиля кулачка, возникающий из-за погрешностей при изготовлении. Поскольку максимальное дополнительное ускорение пропорционально квадрату собственной частоты, даже малый скачок $\Delta П$ может вызвать существенные искажения заданного закона изменения ускорения $\ddot{x}=\Pi^{\prime \prime}\left(\varphi_{1}\right) \omega_{0}^{2}$. Наблюдаемый при этом эффект напоминает езду по булыжной мостовой.

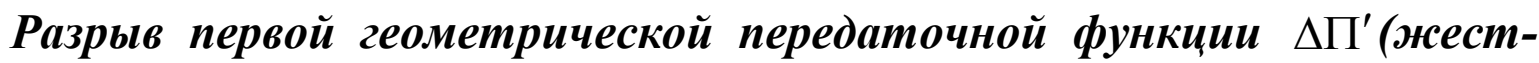
кий удар). Динамический эффект от жесткого удара возрастает с ростом угловой скорости входного звена $\omega_{0}$ и значения собственной частоты. Жесткие удары возникают не только, когда скачком меняется функция П', но и при ударах, сопутствующих выборке зазоров, подключению кинематических цепей с помощью муфт, при фиксации некоторых промежуточных положений выходного звена и в ряде других случаев.

Разрыв непрерывности второй геометрической передаточной функции $\Delta \Pi^{\prime \prime}$ ("мягкий" удар) (рис. 3.1,a). В этом случае максимум дополнительных ускорений, вызванных скачком $\Delta \Pi^{\prime \prime}$, приблизительно равен этому скачку, что приводит к существенному искажению заданных программных 
характеристик. В качестве примера на рис. 3.2, приведены ускорения $\ddot{x}(t)$, изменяющиеся в программном движении по косинусоидальному закону (кривая 1) и $\ddot{y}(t)=\ddot{x}(t)+\ddot{q}(t)$ с учетом возбужденных колебаний (кривая 2$)$.
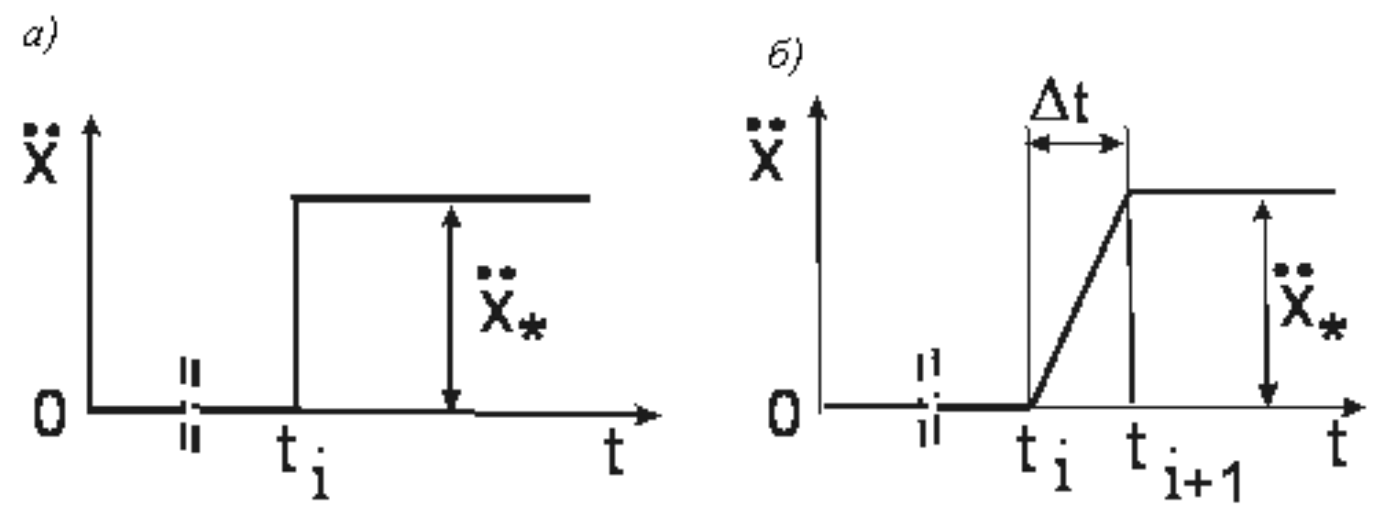

Рис. 3.1. К анализу эффекта от разрыва непрерывности геометрических характеристик

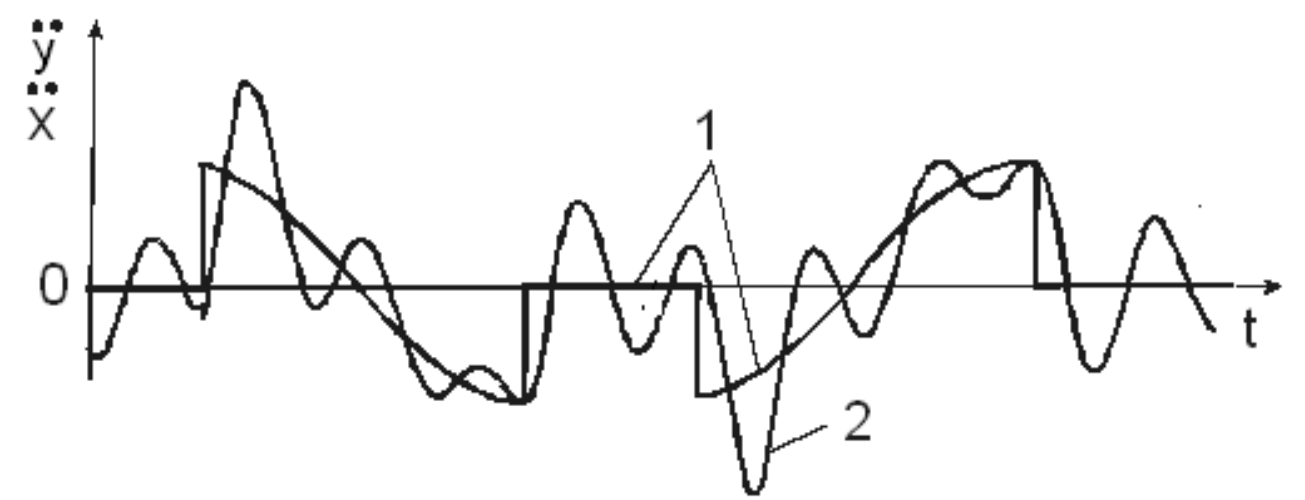

Рис. 3.2. Искажение идеальных ускорений при косинусоидальнои законе изменения ускорений

Разрыв непрерывности третьей геометрической передаточной функции $\Delta \Pi^{\prime \prime \prime}$. В этом случае имеет место скачок производной функции $\dddot{x}(t)=\omega_{0}^{3} \Pi^{\prime \prime \prime}$, называемый ускорением второго порядка или рывком. Поскольку максимальные дополнительные ускорения в отличие от случая «жесткого» удара обратно пропорциональны собственной частоте $k$, повышение жесткости приводит к положительному эффекту.

Резкое изменение функции П" (эквивалентный скачок). Рассмотрим эту задачу на примере резкого изменения ускорений программного движения $\ddot{x}=\Pi^{\prime \prime} \omega_{0}^{2}$ по линейному закону (рис. 3.1, б). Покажем, что при достаточно малом значении $\Delta t=t_{i+1}-t_{i}$ система будет реагировать на изменение $\ddot{x}$ почти так же, как и на скачкообразное изменение этой функции. Сначала определим с помощью зависимости (2.24) сопровождающие колебания $q_{*}$, вызванные на участке $t>t_{i+1}$ двумя скачками $\Delta \Pi^{\prime \prime \prime}-$ при $t=t_{i}$ и при $t=t_{i+1}$ : 


$$
\begin{aligned}
q_{*}= & \Delta q_{i}+\Delta q_{i+1}=D_{i} \exp \left[-n\left(t-t_{i}\right)\right] \sin \left[k\left(t-t_{i}\right)+\alpha_{i}\right]+ \\
& +D_{i+1} \exp \left[-n\left(t-t_{i+1}\right)\right] \sin \left[k\left(t-t_{i+1}\right)+\alpha_{i+1}\right] .
\end{aligned}
$$

Согласно формулам табл.3.1

$$
D_{i}=D_{i+1}=|\Delta \dddot{x}| / k^{3}=\ddot{x}_{*} /\left(k^{3} \Delta t\right) ; \quad \alpha_{i}=0 ; \alpha_{i+1}=\pi .
$$

Отсюда, принимая $t_{i}=0$, имеем

$$
q_{*}=\ddot{x}_{*}\left(k^{3} \Delta t\right)^{-1} \exp [-n(t-\Delta t)][\exp (-n \Delta t) \sin k t-\sin k(t-\Delta t)] .
$$

Ввиду малости $n \Delta t$ в целях упрощения примем $\exp (-n \Delta t)=1$. Тогда

$$
q_{*}=\ddot{x}_{*}\left(k^{3} \Delta t\right)^{-1} \exp [-n(t-\Delta t)][\sin k t(1-\cos k \Delta t)+\cos k t \sin k \Delta t .
$$

Опуская элементарные преобразования, получаем

$$
q_{*}=D_{*} \exp [-n(t-\Delta t)] \sin (k t+\alpha) \text {. }
$$

Здесь $D_{*}$ - эквивалентный скачок, определяемый следующим образом:

$$
D_{*}=\ddot{x}_{*} \kappa^{0}(v) / k^{2},
$$

где $\kappa^{0}(v)=|\sin \pi v| /(\pi v) ; v=\Delta t / T ; T=2 \pi / k$. Отсюда при $t>t_{i+1}$

$$
\left|\ddot{q}_{*}\right|_{\text {max }} \approx k^{2} D_{*}=\ddot{x}_{*} \kappa^{0}(v) \text {. }
$$

При $v=0$ имеем $\kappa^{0}=1$, что соответствует мягкому удару. При $v>0$ соответственно $\kappa^{0}(v)<1$, поэтому этот параметр является показателем смягчения динамического эффекта по сравнению с мягким ударом. Рассмотренному случаю на графике $\kappa^{0}(v)$ (рис. 3.3) отвечает кривая $\delta=0$, что соответствует принятому выше допущению $\exp (-n \Delta t) \approx 1$.

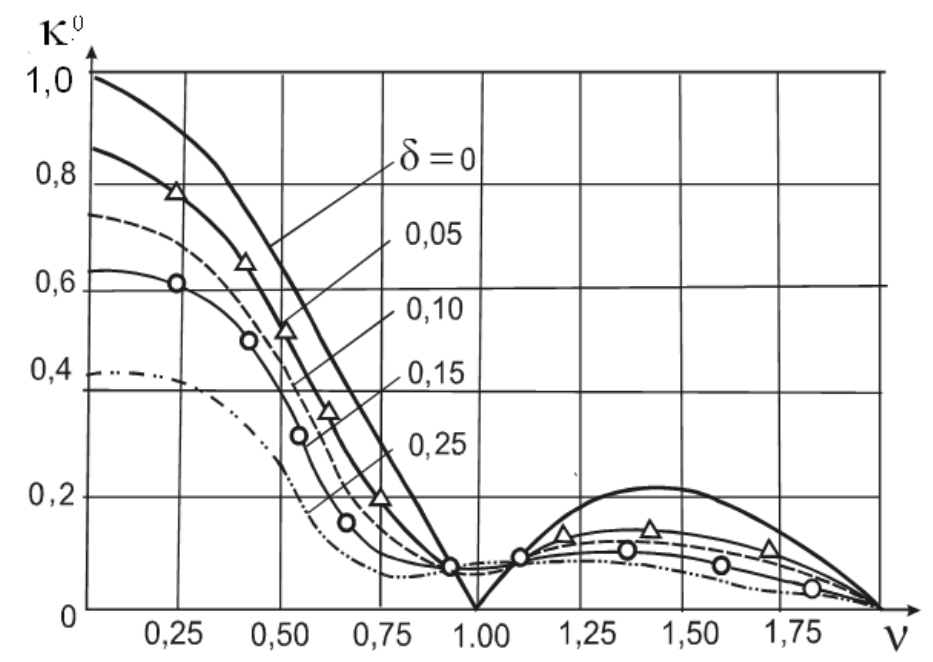

Рис. 3.3. Коэффициент смягчения

Это означает, что в пределах отрезка времени $\Delta t$ мы пренебрегли затуханием колебаний. При $\delta=\lambda /(2 \pi) \neq 0$ ( $\lambda$ - логарифмический декремент) кривые $\kappa^{0}(v)$ располагаются ниже за исключением малых зон в окрестности целых значений 
$v$. Это свидетельствует о том, что силы сопротивления в целом смягчают динамический эффект от резкого изменения идеальных ускорений $\ddot{x}$.

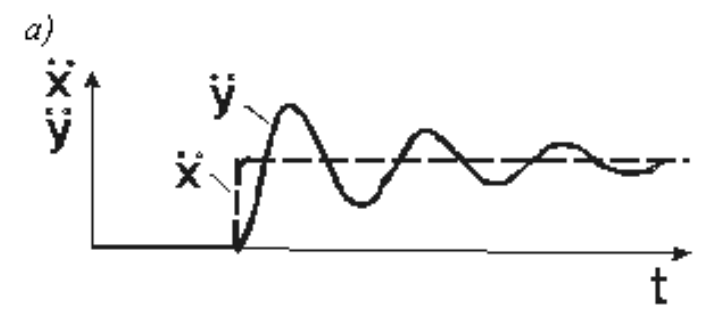

б)
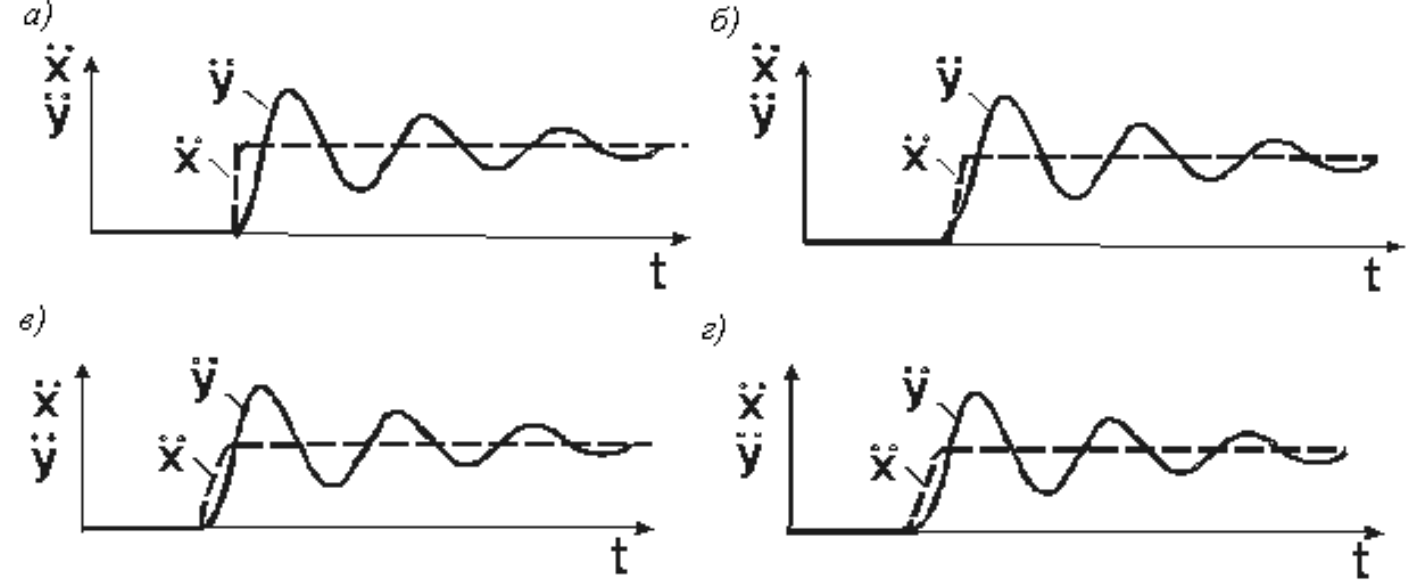

z)

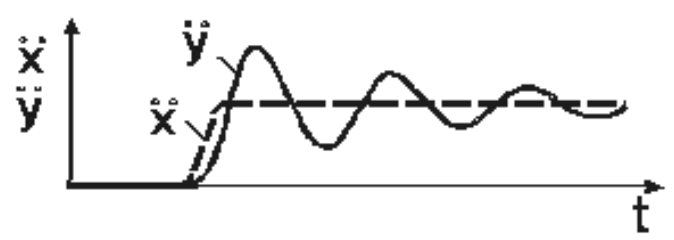

Рис.3.4. Динамический эффект при резких изменениях программных ускорений

Из графиков $\kappa^{0}(v)$ следует, что при малых значениях $v \leq 0,25$ динамический эффект от безразрывного изменения передаточной функиии практически эквивалентен эффекту от скачка. Это иллюстрируется на рис. 3.4 несколькими графиками $\ddot{y}(t)$ при мягком ударе, т.е. при $v=0$ (рис. 3.4, а) и при резком изменении $\ddot{x}(t) \quad(v=0,25)$ для трех случаев: $\ddot{x}(t)$ изменяется по линейному закону (рис. 3.4,б), по закону $\ddot{x}(t)=\ddot{x}_{\max } \sin (0,5 \pi t) / \Delta t \quad$ (рис. 3.4, в) и по закону $\ddot{x}=\ddot{x}_{\max } 0,5(1-\cos (\pi t / \Delta t))($ рис. 3.4, г).

При больших значениях $v$ коэффициент $\kappa^{0}(v)$ резко убывает; при этом $\kappa^{0} \leq 1 /(\pi v)$. На графике $\kappa^{0}(v)$ представляют интерес точки $v=j \pi \quad(j=1,2, \ldots)$, в которых $\kappa^{0} \approx 0$. Эти режимы, отвечающие так называемому квазистатическому нагружению, возникают из-за взаимной компенсации колебаний, вызванных обоими скачками $\Delta \Pi^{\prime \prime}$. Наличие силы сопротивления при этом, однако, приводит лишь к их частичной компенсации.

В приведенных эффектах еще раз проявляется невозможность сведения динамической задачи к геометрической. Другими словами, нельзя предложить закон движения, который был бы оптимальным во всех случаях не зависимо от частотных характеристик механизма.

При принятом законе изменения ускорений «модифицированная трапеция» скачки ускорений отсутствуют. При этом $\Delta \Pi^{\prime \prime}=0$, однако, $\Delta \Pi^{\prime \prime \prime} \neq 0$. Можно показать, что в этом случае

$$
\left|\ddot{q}_{*}\right|_{\max } \approx \Delta w_{*} \kappa^{0}(v)
$$

где $\Delta w_{*}=|w|_{\max }-$ максимальное значение перепада ускорений в программном движении, $\quad v=\Delta \varphi_{i} N /(2 \pi), \quad N=k / \omega ; \quad \Delta \varphi_{i}=s_{i} \varphi_{i} \quad[6,7,8,10]$. 
Согласно (3.5) функция $\kappa^{0}(v)$ определяет максимальное значение дополнительных ускорений от одного «эквивалентного скачка». При $v=0$ имеем $\kappa^{0}=1$, что соответствует мягкому удару. При $v>0$ соответственно $\kappa^{0}(v)<1$, поэтому этот параметр является показателем смягчения динамического эффекта по сравнению с мягким ударом (см. пример). Для закона движения «модифицированная трапеция»

$$
\kappa^{0}=\frac{\sqrt{1+16 v^{2}-8 v \sin 2 \pi v}}{\left|1-16 v^{2}\right|} .
$$

Анализ графика функции $\kappa^{0}(\lambda)$ (рис.3.5) показывает, что при $\lambda<0,25-0,3$ значение $\kappa^{0}$ мало отличается от единицы, а при $\lambda \geq 2,5$ не превосходит 0,13 . Этим условием можно воспользоваться для рационального выбора параметров $s_{j}$ и $N$.

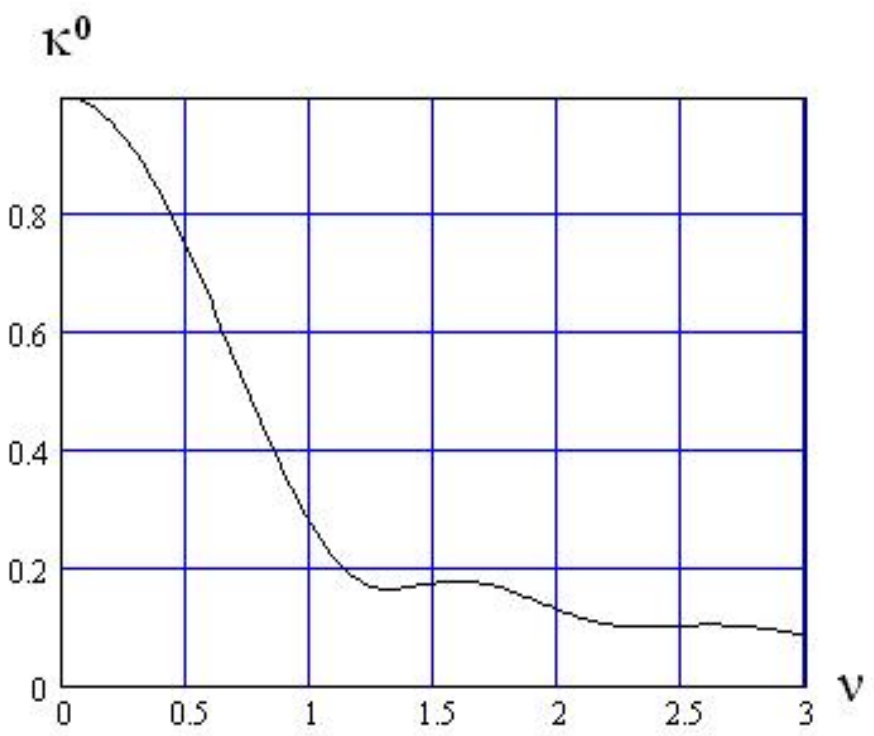

Рис. 3.5. График $\kappa^{0}(v)$

Руководствуясь изложенными выше соображениями, уменьшение в определённой степени максимальных ускорений в кулачковом механизме можно осуществить за счёт коррекции закона движения, На одном периоде $2 \pi$ закон изменения второй передаточной функции П" содержит 8 участков, относительная величина которых определяется параметром $v_{j}$. Зададим условие $v_{j} \geq[v]=2 \div 3$ (см. рис. 3.5 ). Тогда согласно (3.5)

$$
N \geq N_{v}=2 \pi \frac{[v]}{\Delta \varphi_{i} s_{j}} .
$$

Здесь $\Delta \varphi_{i}$ - углы поворота входного звена, соответствующие разбегу и выбегу на прямом и обратном ходе.

Таким образом, условия (3.7), устраняют возможность существенного возбуждения колебаний внутри каждого цикла. Это может быть достигнуто за 
счёт коррекции закона движения, т.е. увеличением параметров $s_{j}$. При этом следует иметь в виду, что на каждом участке $s_{1}+s_{2} \leq 1$.

\section{2. Оптимизация с помощью коррекции частотных характеристик}

Если за счёт геометрических характеристик закона движения не удаётся реализовать приведенные условия, приходится увеличивать параметр $N$. Это можно осуществить уменьшением $\omega$, либо повышением собственной частоты за счет уменьшения массы или момента инерции рабочего органа, либо увеличения жесткости. Разумеется, из этих трех возможностей предпочтительна последняя: в первом варианте мы снижаем производительность, а минимум инерционных характеристик обычно уже заложен при разумном проектировании механизма, поэтому их дальнейшее уменьшение практически невозможHo.

Как уже отмечалось, многие технологические операции осуществляются при постоянной скорости рабочих органов (см. задачу 3). В дополнение к ранее приведенному примеру можно привести нитеводители текстильных машин, рабочие органы металлорежущих автоматов и др. В подобных случаях при определенном выборе параметров модифицированной трапеции можно осуществить безударный реверс рабочих органов [10].

Для рычажных механизмов физические предпосылки возбуждения сопровождающих колебаний совпадают с описанными выше, однако подавление этих колебаний затруднено из-за ограниченных возможностей при синтезе закона движения. В то же время законы движения звеньев рычажных механизмов описываются более «гладкими» функциями, поэтому сопровождающие колебания чаще возникают из-за соударений в зазорах (см. ниже).

Ограничение накопления возмущений. Ввиду периодичности кинематических характеристик с периодом $\tau=2 \pi / \omega$, где $\omega$ - угловая скорость входного звена (кулачка), сумма колебаний, возбужденных от одинаковых скачков, также должна быть периодической функцией. Поэтому для оценки результирующего режима можно снова воспользоваться замкнутой формой решения, основанной на условиях периодичности (см. п. 2.3). При этом согласно формулам (2.31) и (2.33) учет скачков на предыдущих циклах приводит к тому, что скачок $D_{1}$ на рассматриваемом цикле трансформируется в $\mu D_{1}$, где $\mu$ - коэффициент накопления возмущений, а максимальные дополнительные ускорения, вызванные этим скачком, определяя

$$
\sum_{i=1}^{\infty}\left|\Delta \ddot{q}_{1}\right|_{\max }=k^{2} \mu D_{1}
$$

При динамическом синтезе для ограничения уровня сопровождающих колебаний целесообразно ввести ограничение $\mu<[\mu]$, где $[\mu]-$ допускаемое значение коэффициента накопления возмущений. Тогда на основании (2.33) 


$$
1-2 \exp (-\lambda N) \cos 2 \pi N+\exp (-2 \lambda N)<[\mu]^{-2} .
$$

Это неравенство следует решить относительно частотного критерия $N$. Для получения более наглядных результатов примем $[\mu]=1$. Тогда

$$
0,5 \exp (-\lambda N)<\cos 2 \pi N
$$

При $\lambda N \rightarrow 0$ неравенство (3.10) удовлетворяется при $N<E-1 / 6$, либо при $N>E+1 / 6$, где $E$ - целое число. В другом предельном случае, когда $\lambda \rightarrow \infty, \quad N<E-1 / 4$, либо $N>E+1 / 4$. Полученная частотная настройка практически оказывается эффективной лишь при малых значениях $N$ (примерно $N<4 \div 6$ ). Поясним это на простом примере. Пусть $k=170 \mathrm{c}^{-1}$; $\omega=20 \mathrm{c}^{-1}$. При этом $N=k / \omega=8,5$. Это значение соответствует $\mu^{-}<1$. Однако достаточно частоту вращения уменьшить примерно на $1,1 \mathrm{c}^{-1}$ (что в реальных условиях вполне возможно), как $N=9$. Казалось бы, при уменьшении угловой скорости колебания станут менее интенсивными. Но при целых значениях $N$ имеем $\mu=\mu^{+}>1$, следовательно, уровень колебаний повысился.

Таким образом, в этом случае частотный критерий оказался недостаточно надежным. В подобных ситуациях более целесообразно потребовать $\mu^{+}<[\mu]$, принимая $[\mu]=1+[\Delta \mu]$, где $[\Delta \mu]-$ малая положительная добавка (например $[\Delta \mu]<0,05 \div 0,1)$. Тогда на основании (2.33)

$$
N>\lambda^{-1}\{\ln (1+[\Delta \mu]) /[\Delta \mu]\} .
$$

При $\lambda N>3$ имеем $0,96<\mu<1,04$; это означает, что возбуждаемые сопровождающие колебания практически оказываются задемпфированными за время одного оборота входного звена. Заметим, что учет ограничения $\mu<[\mu]$ является весьма желательным даже при устранении явных причин возбуждения сопровождающих колебаний, поскольку из-за случайных факторов всегда имеют место периодические возмущения, не учтенные в инженерном расчете, которые при больших значениях $\mu$ могут существенно увеличить интенсивность колебаний.

\section{3. Оптимизационный синтез законов движения с использованием компьютерного моделирования}

В параграфах 3.1 и 3.2 была изложена методика подавления колебаний, возникающих при скачкообразных и резких изменениях геометрических и динамических характеристик механизмов. Оптимизация этих характеристик представляет собой многокритериальную задачу, поэтому для уменьшения трудоемкости при инженерных расчетах целесообразно воспользоваться компьютерным моделированием.

Эволюция колебаний при изменении параметров показана на рис.3.6 


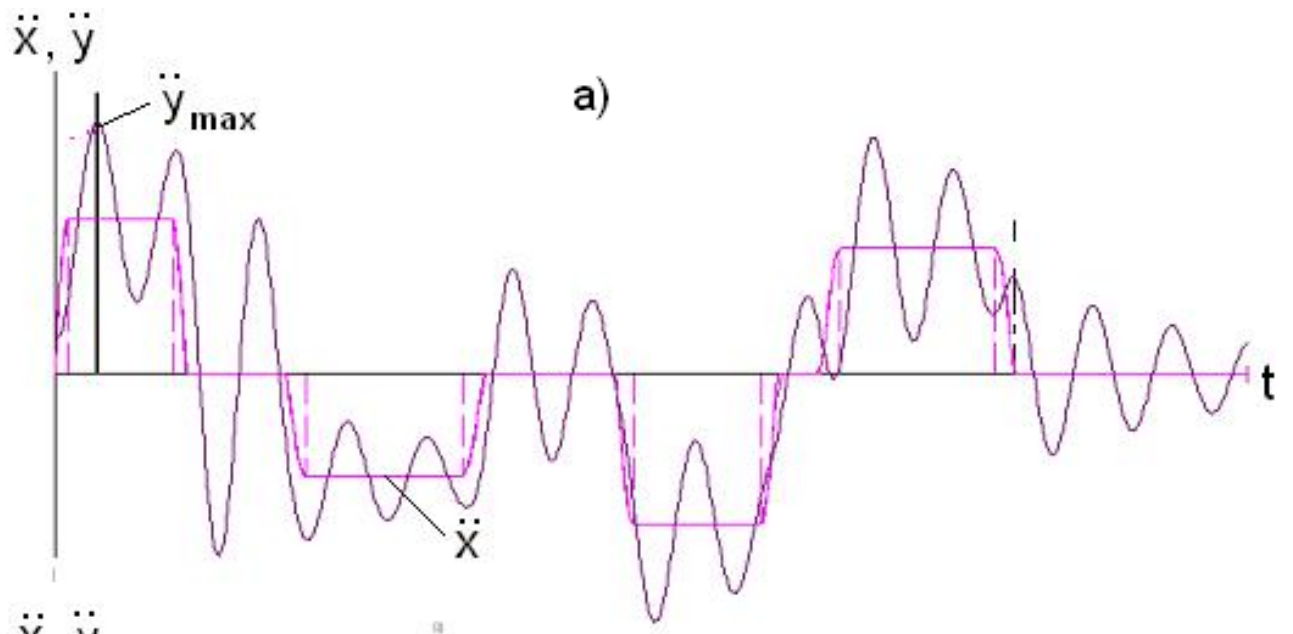

$\ddot{x}, \ddot{y}$

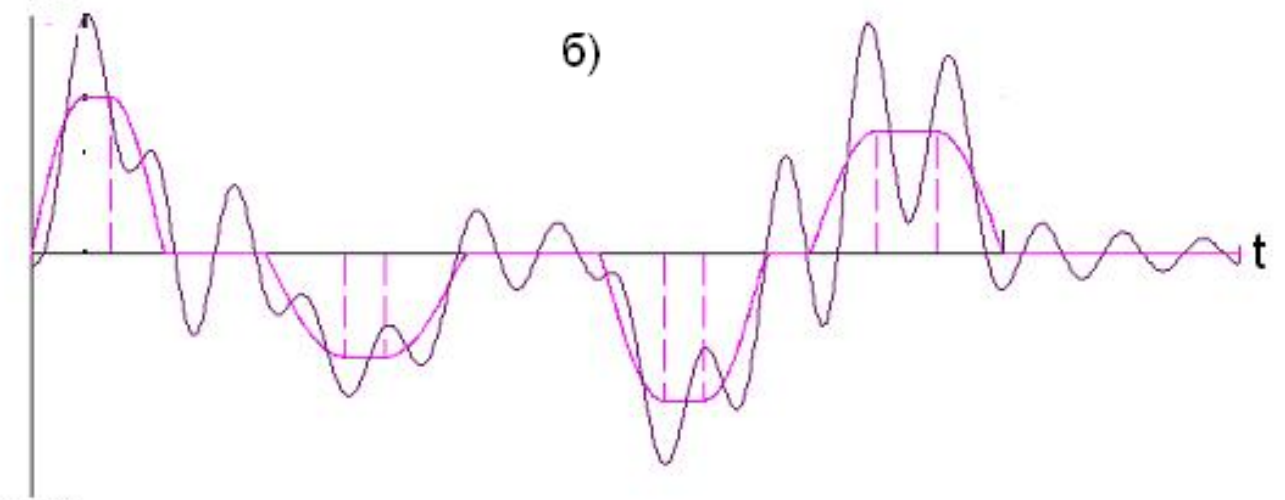

$\ddot{x}, \ddot{y}$

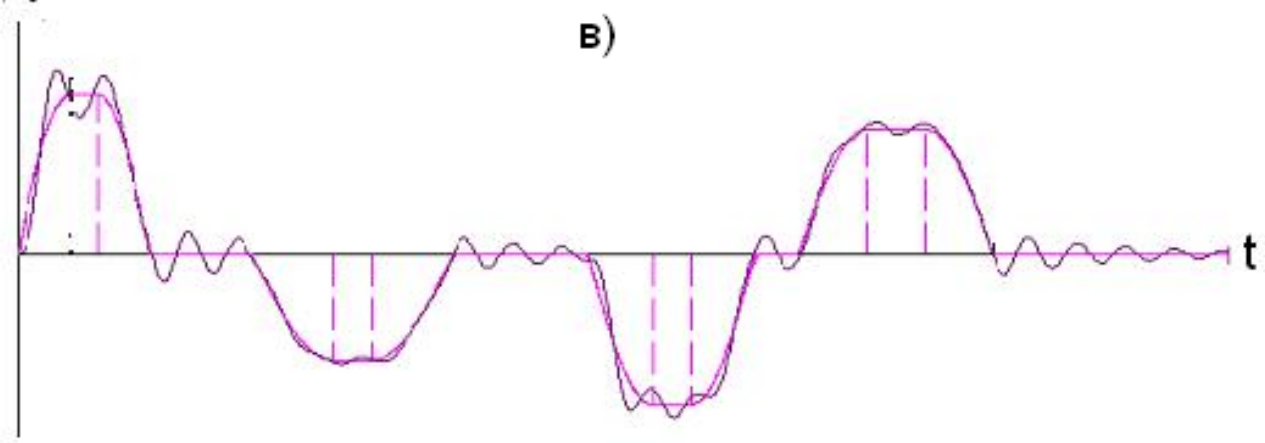

Рис. 3.6 Оптимизация закона движения при коррекции геометрических и динамических характеристик

Оптимизация геометрических характеристик изложена в п. 3.1 и соответствует трансформации перехода от режима, показанного на рис. 3.6, а $\left(s_{i}=0,1\right)$, к режиму - на рис.3.6,б $\left(s_{i}=0,4\right)$. Нередко этого достаточно, чтобы снизить уровень колебаний до приемлемых значений. В нашем случае имеет место лишь некоторое незначительное уменьшение колебаний, поэтому дополнительно произведем коррекцию частотных характеристик (см. п. 3.2). Переход от режима, показанного на рис. 3.6,б к режиму на рис.3.6,в соответствует увеличению $N=k / \omega$ от 15 до 25 . Разумеется, реализация этой коррекции следует произвести не за счет понижения производительности (параметр $\omega)$, а увеличением жесткости. Эффективность полученного результата оче- 
видна. В Приложении 1 представлена соответствующая компьютерная программа [24].

\section{4. Динамические ошибки при кинематическом возбуждении исполнительных органов повышенной протяжённости}

Во многих технологических машинах рабочие органы установлены на валах или стержнях, совершающих заданное неравномерное программное движение. На это движение накладываются нежелательные колебания, вызванные кинематическим возмущением, причем, как показывает анализ, особенно значительные динамические ошибки возникают из-за виброускорений

Динамическая модель. Представленная на рис.3.7 динамическая модель состоит из двух упругодиссипативных элементов, разделенных элементом, имитирующим функцию положения выходного звена циклового механизма $\varphi_{1}=\Pi(\varphi)$ и исполнительного органа, схематизированного в виде диска и вала с моментами инерции $J_{1}$ и $J_{2}$. Примем следующие условные обозначения: $\omega, \varphi=\omega t-$ угловая скорость и координата входного звена; $c_{0}, c_{1}-$ коэффициенты жесткости; $\psi_{0}, \psi_{1}$ - коэффициенты рассеяния; $G$ - модуль сдвига; $I$ - полярный момент инерции.

Используя условия энергетического баланса, приведем упругодиссипативные характеристики $c_{0}, \psi_{0}$ к оси исполнительного органа. При этом примем $d \Pi / d \varphi=r_{0} \sin \varphi$, что соответствует распространенному случаю программного движения выходного звена. Тогда приведенные коэффициенты жесткости и рассеяния определяются как

$$
c=c_{1}\left(1+\chi \sin ^{2} \varphi\right)^{-1} ; \psi=\left(\psi_{0} c_{1} / c_{0}+\psi_{1}\right)\left(1+\chi \sin ^{2} \varphi\right)^{-1},
$$

где $\chi=r^{2} c_{1} / c_{0}$.
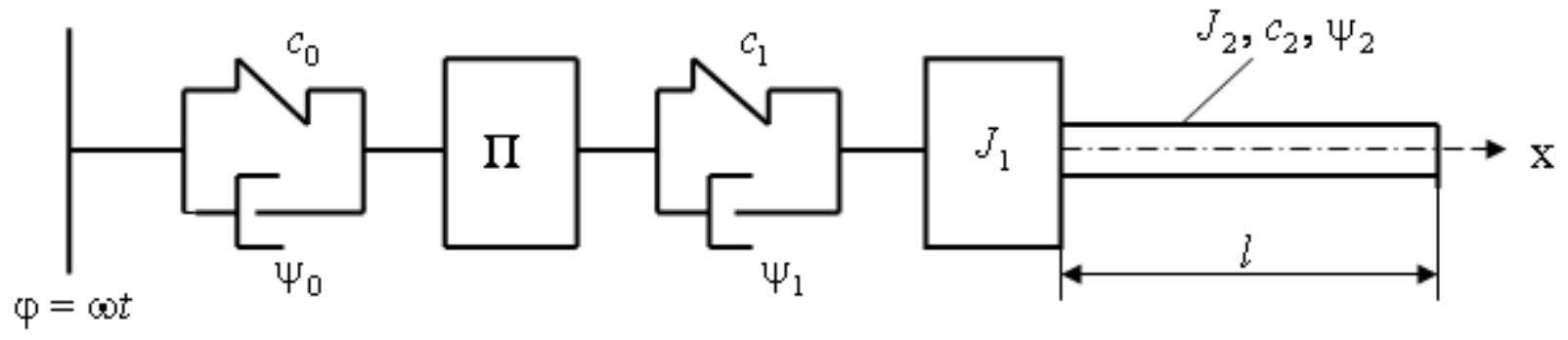

Рис. 3.7. Динамическая модель

Дифференциальное уравнение колебаний исполнительного органа в координатной системе $\varphi_{1}=\Pi(\varphi)$ имеет вид

$$
G I \frac{\partial^{2} u}{\partial x^{2}}-\rho \frac{\partial^{2} u}{\partial t^{2}}-b \frac{\partial u}{\partial t}=\rho \omega^{2} \frac{d^{2} \Pi}{d \varphi^{2}}-M(x, t),
$$

где $u=\varphi_{2}(x, t)-\Pi(\varphi)-$ динамическая ошибка в сечении $x ; \rho=J_{2} / l-$ погонный момент инерции; $b$ - эквивалентный коэффициент линейной силы сопротивления (см. ниже); $M(x, t)$ - интенсивность распределенного внешнего момента. 
Согласно (3.12) приведенные упругодиссипативные характеристики передаточного механизма являются функциями «медленного» времени $\tau=\varphi / \omega$. При этом граничные условия определяются следующим образом:

$$
G I \frac{\partial u}{\partial x}(0)=c(\tau) u(0)+b(\tau) \frac{\partial u}{\partial t}(0)+J_{1} \frac{\partial^{2} u}{\partial t^{2}}(0) ; \quad \frac{\partial u}{\partial x}(0)=0 .
$$

Частотный и модальный анализ. Принимая во внимание пренебрежимо малое влияние сил сопротивления на собственные частоты и формы колебаний, на этом этапе примем $b \equiv 0$. Согласно методу условного осциллятора $[4-11,13,14]$ решение однородного дифференциального уравнения, полученного из (3.12) при обращении в нуль правой части, ищем в форме

$$
u=X(x, \tau) \cos \Phi(t),
$$

где $\tau$ - медленное время.

В одном из сечений (например, при $x=\ell$ ) функции $X(\ell, \tau)$ и Ф согласно методу условного осциллятора могут быть связаны дополнительным условием

$$
2 \frac{d B}{d \tau} p+B \frac{d p}{d \tau}=0,
$$

где $p(\tau)=d \Phi / d t-$ «собственная» частота; $B=X(\ell, \tau)$.

На основании $(3.13) \div(3.16)$ формальное частотное уравнение может быть записано в виде

$$
\theta(\tau)[\theta(\tau)+\mu \operatorname{tg} \theta(\tau)]=\sigma_{0}^{2}\left(1+\chi \sin ^{2} \omega \tau\right)^{-1},
$$

где $\mu=\rho l / J_{1} ; \sigma_{0}=k_{1} / k_{2} ; k_{1}=\sqrt{c_{1} / J_{1}} ; k_{2}=\sqrt{G I /\left(J_{2} l\right)} ; \theta_{r}(\tau)=p_{r}(\tau) / k_{2}$.

Уравнение (3.17) имеет неограниченное множество корней $\theta_{r}(\tau)$ $(r=\overline{1, \infty})$, которым соответствуют «собственные» частоты $p_{r}(\tau)=k_{2} \theta_{r}(\tau)$.

Если принять $B=1$ при $\tau=0$, то нестационарная форма колебаний определяется следующей зависимостью:

$$
X_{r}(x, \tau)=\sqrt{\frac{\theta_{r}(0)}{\theta_{r}(\tau)}} \cos \left[\Phi_{r}(\tau)(1-x / l)\right],
$$

где $r$ - номер частоты

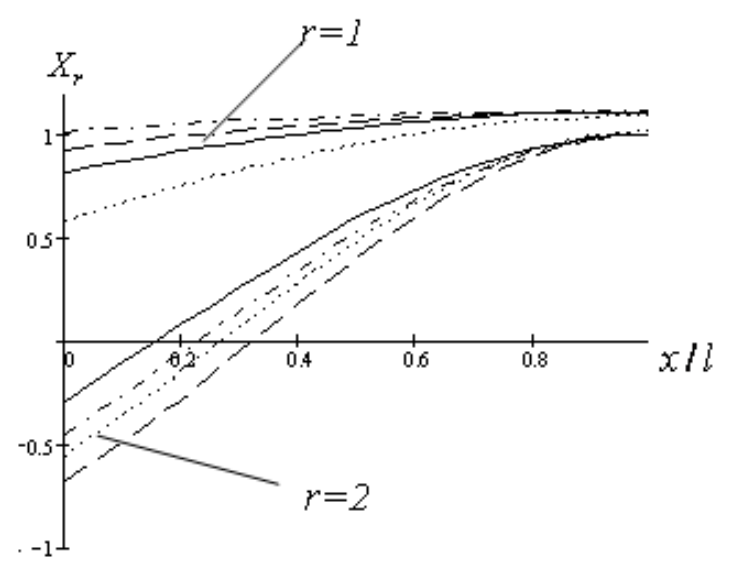

Рис. 3.8. Эволюция форм колебаний 
Эволюция форм колебаний при $\chi=1, \varphi=\pi / 2$ и варьировании параметрами $\mu$ и $\sigma_{0}$ показана на рис. 3.8. При этом сплошная линия отвечает $\mu=0,5, \sigma_{0}=1$, штриховая $-\mu=3, \sigma_{0}=1$, штрих пунктирная $-\mu=1, \sigma_{0}=0,5$, пунктирная $-\mu=1, \sigma_{0}=2$.

Анализ динамических ошибок. Используя аппарат квазинормальных координат, представим решение неоднородного дифференциального уравнения (3.13) как

$$
u=\sum_{r=1}^{\infty} X_{r}(x, \tau) U_{r}(t),
$$

где функция $U_{r}$ определяется из следующего дифференциального уравнения:

$$
\ddot{U}_{r}+2 n_{r}(\tau) \dot{U}_{r}+p_{r}^{2}(\tau) U_{r}=W_{r}(t) .
$$

В левую часть этого уравнения включен опущенный при частотном анализе диссипативный член, для определения которого можно воспользоваться следующей зависимостью, полученной при исключении диссипативных связей между различными формами колебаний :

$$
\psi_{r}^{*}(\tau)=\frac{c(\tau) l \psi(\tau)+G I D_{r}(\tau) \psi_{r}}{c(\tau) l+G I D_{r}(\tau)}
$$

где $D_{r}=l^{-1} \int_{0}^{l} X_{r}^{\prime 2}(x, \tau) d x=0,5 \theta_{r}(\tau)\left[\theta_{r}(\tau)-0,5 \sin 2 \theta_{r}(\tau)\right] ; \quad \psi, \psi_{r}-$ коэффициенты рассеяния соответственно для обеих рассматриваемых подсистем; $\psi_{r}^{*}-$ приведенный к форме $r$ коэффициент рассеяния. При этом $n_{r}(\tau) \approx \psi_{r}^{*}(\tau) p_{r}(\tau) /(4 \pi)$.

Правая часть дифференциального уравнения (3.20) может быть найдена как

$$
W_{r}=-w_{*}(t) \int_{0}^{l} X_{r} d x / \int_{0}^{l} X_{r}^{2} d x=-w_{*}(t) H_{r}(\tau), \quad \text { где } H_{r}(\tau)=\frac{2 \sin \theta_{r}(\tau)}{\theta_{r}(\tau)+0,5 \sin 2 \theta_{r}(\tau)} .
$$

На основании метода условного осциллятора частное решение уравнения (3.20) при медленном изменении коэффициентов имеет вид [5-11]

$$
U_{r}=\frac{1}{\sqrt{p_{r}(t)}} \int_{0}^{t} \frac{W_{r}(z)}{\sqrt{p_{r}(z)}} \exp \left[-\int_{z}^{t} n_{r}(\xi) d \xi\right] \sin \left[\int_{z}^{t} p_{r}(\xi) d \xi\right] d z .
$$

Для инженерных оценок представляет интерес функция $\ddot{u}_{\max }(x / \ell)$, характеризующая распределение дополнительных ускорений вдоль оси $x$. Следует отметить, что при реальных значениях параметров именно виброускорения, вызванные резкими изменениями ускорений программного движения $d^{2} \varphi_{1} / d t^{2}$, являются источником повышенной виброактивности, лимитирующим эксплуатационные характеристики машин.

Как показывает анализ приведенных зависимостей, при изменении заданного программного ускорения от 0 до $w_{\max }$ за время $\Delta t$

$$
\ddot{u}_{\max }=w_{\max } \sum_{r=1}^{\infty} \kappa_{r}\left(v_{r}\right) H_{r} X_{r},
$$


где $v_{r}=\Delta t / T_{r} ; T_{r}=2 \pi / \bar{p}_{r} ; \bar{p}_{r}-$ усредненное значение $p_{r}(\tau)$ на интервале $[0, \Delta t]$, $w_{\max }$ - максимальное значение $|w(t)|$; определение функции $\kappa_{r}$ см. п. 3.1.

Поскольку при принятом нормировании форм $X_{r}(\ell)=1$, из зависимости (3.23) следует, что сумма $K=\sum_{r=1}^{\infty} \kappa_{r}\left|H_{r}\right|$ может служить критерием для оценки уровня динамической ошибки по виброускорениям. Слагаемые этой суммы $K_{r}=\kappa_{r}\left|H_{r}\right|$ характеризуют распределение ускорений по различным формам колебаний. Наиболее существенный вклад вносит член $K_{1}$.

Можно показать, что $v_{r}=\Delta \varphi_{1} k_{2} \bar{\theta}_{r} /\left(2 \pi \omega \sigma_{0}\right)$, где $\Delta \varphi-$ угол поворота главного вала, отвечающий изменению $w(t)$ между экстремумами, $\bar{\theta}$-среднее значение $\theta$. При малых значениях $v_{r}$ критерий $K_{1}$ мало отличается от единицы. Следовательно, $\ddot{u}_{\max } \approx w_{* \max }$, что отвечает так называемому мягкому удару. С ростом параметра $\theta$, пропорционального «собственной» частоте, этот критерий резко убывает. Интересно, что при некоторых малых значениях $v_{1}$ критерий имеет максимум, превышающий по величине единицу. Это, однако, не означает, что максимальное дополнительное ускорение $\ddot{u}_{\max }$, возникающее от единичного скачка $w_{*}(t)$, может стать больше $w_{* \max }$, так как критерий $K_{1}$ характеризует лишь составляющую, отвечающую первой форме, которая при суммировании по формуле (3.23) компенсируется за счет остальных форм.

При формировании критерия $K$ обычно следующим по значимости является член $K_{3}$. Относительно малое влияние членов, отвечающих четным формам, связано с близостью к антисимметричной форме колебаний, для которой интегральное по длине $\ell$ значение элементарной работы от заданной симметричной нагрузки относительно мало.

Для анализа характера распределения виброускорений по оси $x$ введем в рассмотрение функцию

$$
Z_{1}=K_{1}(v) X_{1}(x / l) / X_{1}(0),
$$

которая отвечает низшей форме колебаний. На рис.3.9 приведены графики этой функции, полученные при $\chi=1, \mu=1$ для двух значений $v_{1}=0,1$ и $v_{1}=1$.

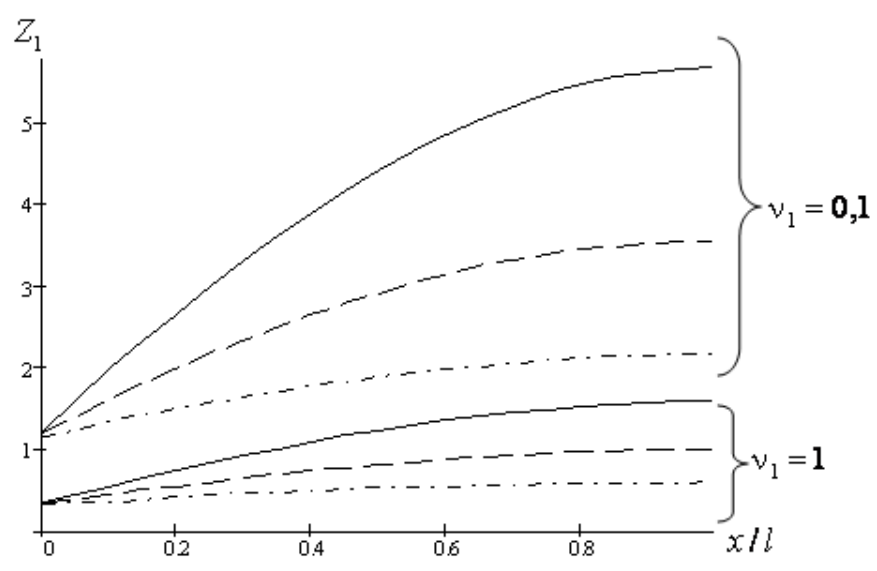

Рис. 3.9. Распределение виброускорений 
При этом сплошные линии соответствуют $\sigma_{0}=4$, штриховые $-\sigma_{0}=3$, штрихпунктирные $-\sigma_{0}=2$. Как следует из графиков, с уменьшением параметра $\sigma_{0}$ ускорения более равномерно распределяются по длине исполнительного органа.

Для контроля уровня виброактивности определим нагрузку, передаваемую на привод $c(\tau) u(0, t)=c(\tau) \sum_{r=1}^{\infty} X_{r}(0, \tau) U_{r}(t)$. Критерием оценки каждого из слагаемых суммы, входящего в эту зависимость служит $N_{r}=\left|X_{r}(0, \tau) U_{r}\right|_{\max }$.

Анализ этого критерия свидетельствует, что удовлетворение условий $v_{1} \geq 3 \div 4$ также представляется целесообразным и с позиций снижения виброактивности системы. Рассмотрение других законов изменения ускорений программного движения приводит к аналогичным выводам.

\section{5. Минимизация ускорений на участках кратковременного реверса рабочих органов машин}

Поставим следующую задачу оптимизации закона движения: при заданном перепаде ускорений $\Delta \omega=\omega^{2}\left|\Pi^{\prime \prime}\right|_{\max }$, соответствующем отрезку времени $\Delta t=t_{1}-t_{0}$, найти такую функцию П", при которой дополнительные колебания $q$, возбужденные на этом отрезке, были бы равны нулю при $\mathrm{t}>\mathrm{t}_{1}$. Эта задача относится к классу вариационных. Имея в виду необходимость учета ряда ограничений, в данном случае целесообразно воспользоваться так называемым полидинамическим методом, являющимся одной из модификаций прямого метода Релея-Ритца [2,19,21].

Используя подстановку Эйлера $q=q_{1} \exp \left[-n\left(t-t_{0}\right)\right], \quad$ где $n=\lambda k /(2 \pi), \lambda-$ логарифмический декремент, представим уравнение (2.2) в следующем виде:

$$
\ddot{q}_{1}+k_{1}^{2} q=w_{1}(t)
$$

где $k_{1}^{2}=k^{2}-n^{2} ; w_{1}=w \exp \left[n\left(t-t_{0}\right)\right]$.

Таким образом, заданному перепаду $\Delta \omega$ в новых координатах отвечает перепад $\Delta w_{1}=\Delta w e^{n \Delta t}$. Однако при малых отрезках времени $\Delta t$, соизмеримых с периодом колебаний, экспоненциальный множитель близок к единице, поэтому обычно $\Delta w_{1} \approx \Delta w$.Решение поставленной задачи приводит к следующим условиям: 


$$
\left.\begin{array}{c}
k\left|\int_{t_{0}}^{t_{0}+\Delta t} \xi(u) \sin k(t-u) d u\right|=1 ; \\
\int_{t_{0}}^{t_{0}+\Delta t} \xi(u) \cos k(t-u) d u=0,
\end{array}\right\}
$$

где $\xi(t)=\left[w_{1}(t)-w_{1}\left(t_{0}\right)\right] / \Delta w_{1}$.

Проиллюстрируем решение этой задачи на примере изменения ускорений по закону «модифицированная трапеция» (см. гл.1). Условиям (3.25) отвечает множество функций $\xi(t)$. Выбор одной из них определяет функцию $\Pi^{\prime \prime}(\varphi)$ на рассматриваем участке $\Delta \varphi=\omega \Delta t$, а следовательно, и функцию $\theta^{\prime \prime}(\tau)$ при $1-s_{2} \leq \tau \leq 1$. Ниже приводится одно из возможных решений данной задачи.

$$
\begin{gathered}
\theta^{\prime \prime}=\left\{\begin{array}{lr}
\theta^{\prime \prime} \max & \left(0 \leq \tau \leq 1-s_{2}\right) ; \\
\theta^{\prime \prime} \max \left(1-\zeta \cos \pi \frac{1-\tau}{s_{2}}\right) & \left(1-s_{2} \leq \tau \leq 1\right) ;
\end{array}\right. \\
\theta^{\prime}=\left\{\begin{array}{lr}
\theta_{\max }^{\prime \prime} \tau & \left(0 \leq \tau \leq 1-s_{2}\right) ; \\
0,5 \theta_{\max }^{\prime \prime}\left(\tau+\frac{\zeta s_{2}}{\pi} \sin \pi \frac{1-\tau}{s_{2}}+1-s_{2}\right) & \left(1-s_{2} \leq \tau \leq 1\right) ;
\end{array}\right. \\
\theta=\left\{\begin{array}{lr}
0,5 \theta_{\max }^{\prime \prime} \tau^{2} & \left(0 \leq \tau \leq 1-s_{2}\right) ; \\
0,5 \theta_{\max }^{\prime \prime}\left[0,5 \tau^{2}+\left(1-s_{2}\right) \tau+\frac{\zeta s_{2}^{2}}{\pi^{2}} \cos \frac{1-\tau}{s_{2}} \pi-\right. & \left(1-s_{2} \leq \tau \leq 1\right) . \\
\left.-0,5\left(1-s_{2}\right)^{2}+\zeta s_{2}^{2} / \pi^{2}\right] &
\end{array}\right.
\end{gathered}
$$

$$
\text { Здесь } \theta_{\max }^{\prime \prime}=2\left[1-0.5 S_{2}^{2}\left(1-4 \zeta / \pi^{2}\right)\right]^{-1} ; \zeta=1-0,25 v^{-2} \text {. }
$$

Таким образом, зависимости (3.26) определяют функцию положения и ее производные при оптимальной частотной настройке. Следует подчеркнуть, что полученный результат еще раз свидетельствует о том, что задача об оптимальных законах движения не может в общем случае базироваться лишь на геометрических и кинетостатических представлениях. Физический смысл оптимальной частотной настройки состоит в том, что при $t>t_{0}+\Delta t$ начальные 
условия, связанные с возбуждением колебаний на отрезке времени $\Delta t$, обращаются в нуль.

Использование законов движения с оптимальной частотной настрой кой позволяет существенно уменьшить максимально допускаемое значение $v_{\min }$. Однако с уменьшением $v$ растет чувствительность функции $\kappa(v)$ к ошибке $\Delta v$. При этом

$$
\kappa\left(v_{*}+\Delta v\right) \approx \frac{2}{v_{*}}\left|\frac{\cos \pi v_{*}}{4 v_{*}^{2}-1}\right| \Delta v,
$$

где $v_{*}$ - отвечает оптимальной частотной настройке $\left(\kappa\left(v_{*}\right)=0\right)$.

Анализ показывает, что при $v_{*}=1,5$ коэффициент при $\Delta v$ в формуле (3.27) обращается в нуль; при $v_{*}=1$ имеем $\kappa(v)=2 \Delta v / 3$, а при $v_{*}=0,5$ после раскрытия неопределенности получаем $\kappa(v)=\pi \Delta v$. Таким образом, уменьшать минимальное значение $v$ ниже $0,5 \div 07$ представляется нецелесообразным, поскольку при этом требуется повышенная точность исходной информации и соблюдение жестких требований при практической реализации законов движения. Однако, даже при отмеченных ограничениях параметр $N=k / \omega$ может быть уменьшен в несколько раз, что для фиксированных упругих и инерционных характеристик системы позволяет соответственно повысить допускаемое значение угловой скорости входного звена $\omega$.

На рис.3.10 приведены результаты компьютерного моделирования колебаний, возбуждаемых на участке реверса в конце прямого и начале обратного хода при $v=0,5$, программном изменении ускорений по закону модифицированной трапеции (рис,3.10,a) и синтезированном выше оптимальном законе (рис.3.10,б). Сопоставление ускорений $w(t)$ (кривые I) свидетельствует о том, что при изменении ускорений по закону модифицированной трапеции возбуждаются интенсивные колебания, за счет которых максимальные ускорения повышаются на $63 \%$, в то время как при оптимальном законе лишь на 4\%.
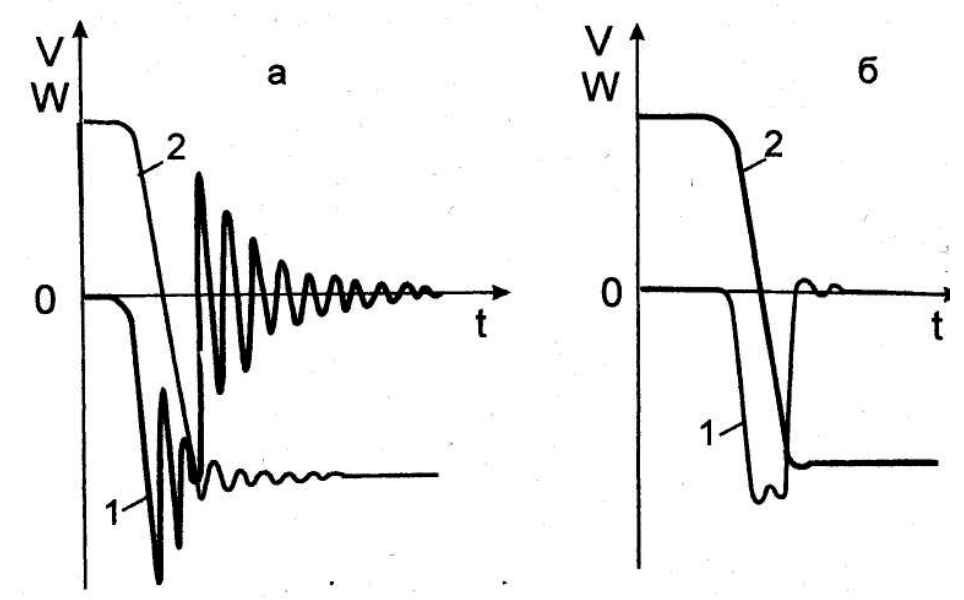

Рис.3.10. К анализу результатов оптимизации 
Кроме того, в первом случае имеют место динамические ошибки по скорости $\mathrm{v}$ на участке равномерного движения, а во втором - они практически отсутствуют (кривые 2). Эти результаты свидетельствуют об эффективности разработанной методики.

\section{6. Инженерные рекомендации при синтезе законов движения кулачковых механизмов с учетом возбуждаемых колебаний}

На основании приведенного выше анализа могут быть сформулированы следующие условия, исключающие возможность существенного искажения заданных программных кинематических характеристик.

1. В функции положения П( $\varphi)$, а также в первой и второй передаточных функциях $\Pi^{\prime}(\varphi)=d \Pi / d \varphi$ и $\Pi^{\prime \prime}(\varphi)=d^{2} \Pi / d \varphi^{2}$ должны быть устранены разрывы непрерывности.

2. Так как при малых значениях $\lambda=\Delta t / T(\lambda<0,25)$ плавное изменение ускорений практически эквивалентно мягкому удару, следует принять (с запасом) $\Delta t>(2 \div 3) T$, где $T=2 \pi / k-$ период свободных колебаний.

3. В целях ограничения динамического эффекта от колебаний, возбужденных на предыдущих циклах движения, следует ограничить максимальное значение коэффициента накопления возмущений $\mu^{+}$. В частности при $[\mu]<1,1$ необходимо потребовать, чтобы $N=k / \omega>2,4 \lambda^{-1}$. Если, например, $\lambda=0,2$, то собственная частота $k$ должна по меньшей мере в 12 раз превышать угловую скорость кулачка $\omega_{0}$.

В процессе рационального динамического синтеза законов движения с учетом колебаний возникает задача с противоположными тенденциями влияния длительности переходного участка диаграммы ускорений, определяемой параметром $s_{j}$. С одной стороны, при увеличении $s_{j}$ возрастает значение $\left|\Pi^{\prime \prime}\right|_{\max }$ за счет уменьшения коэффициента заполнения) а, с другой - в среднем понижается $\kappa^{0}(\lambda)$. В подобных случаях окончательно выбрать этот параметр можно, обеспечивая минимальное значение $\left(1+\mu \kappa^{0}\right)\left|\Pi^{\prime \prime}\right|_{\max }$.

Следует, однако, отметить, что диапазон возможного управления за счет параметра $s_{j}$ относительно невелик $\left(s_{j} \approx 0,15 \ldots 0,35\right)$. Опираясь на зависимость (2.33), можно формально прийти к выводу, что в функции ускорений выходного звена следует по возможности устранить скачок производных как можно более высокого порядка. Известны различные законы движения, описываемые полиномами, в которых такое требование удовлетворяется вплоть до производных пятого порядка и выше. Однако при практическом использовании таких законов возникают, по крайней мере, две опасности. Во-первых, устранение скачка производной не исключает возможность возникновения эквивалентного эффекта при резком изменении этой производной. Это отразится в соответствующем значении эквивалентного скачка (см. выше), причем, 
чем выше порядок производной функции П(ф), обращающейся в нуль в начале и конце фазы движения, тем в большей степени график функции П(ф) «стелется» при подходе к крайним точкам (рис. 3.11). Представим себе, что точность изготовления определяет некоторые «полоски» шириной $\Delta$, в пределах которых значение функции П не гарантируется.

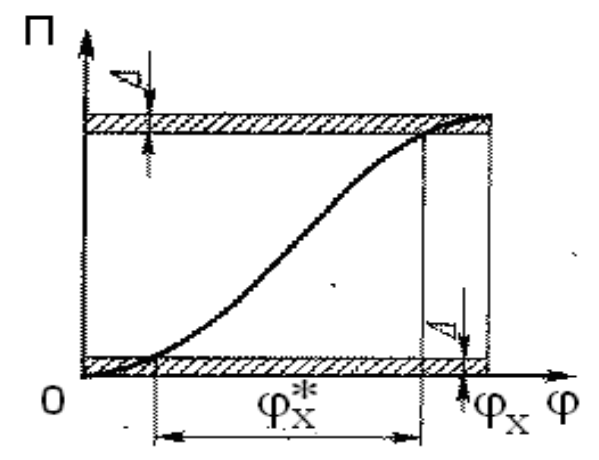

Рис. 3.11. К анализу динамических характеристик в окрестности выстоев

Тогда принципиально возможно, что угол поворота, соответствующий рабочему ходу, может сократиться от $\varphi_{x}$ до $\varphi_{x}^{*}$. Это может не только привести к существенному увеличению значения $\Pi_{\max }^{\prime \prime}$, которое обратно пропорционально квадрату соответствующего фазового угла (см. табл. 1.2), но и к ударам в начале и конце цикла. Отсюда следует вывод: чем больше плавность подхода

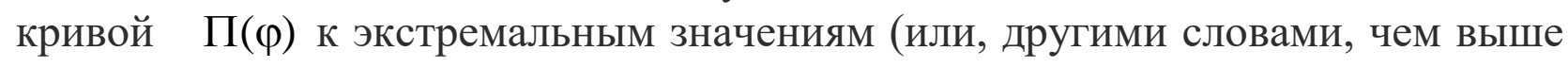
порядок производной, обращающейся в этих точках в нуль), тем более жесткими должны быть технологические требования к точности воспроизведения заданных характеристик. В противном случае создается лишь ложная и весьма вредная иллюзия оптимального решения динамической задачи. Этот пример является еще одним наглядным подтверждением важности комплексного подхода к решению современных задач механики машин.

В современных машинах широко используются механизмы с неудерживающими связями, в которых для предотвращения разрывов контакта в кинематических парах применяется силовое замыкание, реализуемое с помощью пружин. С пружинным замыканием чаще всего приходится сталкиваться в кулачковых механизмах, однако нередко замыкающие пружины устанавливаются и на звеньях рычажных, кулачково-рычажных и других механизмов с целью частичного или полного устранения пересопряжения рабочих поверхностей кинематических пар, возникающих при перекладе в зазоре (см. гл. 4)

\section{7. Динамическая разгрузка}

Общие сведения о разгружающих устройствах. Как уже отмечалось выше, один из способов уменьшения или перераспределения сил, действующих на звенья или реакции в кинематических парах, связан с установкой специальных разгружающих устройств. Выше показано, что основным источником вибраций являются переменные вынуждающие силы, которые обусловлены не только выполняемым технологическим процессом, но и большими 
инерционными нагрузками, возникающими при заданном программном движении звеньев. С помощью разгружающих устройств стремятся уменьшить или, по крайней мере, «сгладить» функцию возмущения. Использование таких устройств, как показывает инженерная практика, нередко является весьма эффективным средством снижения виброактивности механизмов и износа элементов кинематических пар, что, в свою очередь, повышает надежность и долговечность машин [10, 13, 22, 28, 32].

В простейшем случае роль динамического разгружателя (ДР) может выполнять обыкновенная пружина. В этом легко убедиться на следующем примере. Пусть выходное звено механизма массой $m$ перемещается по закону $y(t)=y_{0} \cos \omega t$ (рис.3.12).

a)

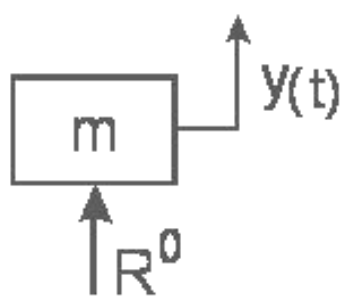

6)

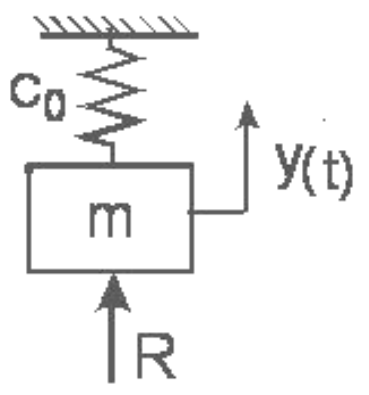

Рис. 3.12. К анализу реакций при динамической разгрузке

Если установить между выходным звеном и корпусом пружину, то $m \ddot{y}=R-c_{0} y$, где $c_{0}$ - коэффициент жесткости пружины; $R-$ проекция реакции со стороны механизма, играющая роль движущей силы. После подстановки $y(t)$ имеем $R=\left(m \ddot{y}+c_{0} y\right)=\left(c_{0}-m \omega^{2}\right) y_{0} \cos \omega t$. Очевидно, что $R=0$ при $\omega=\omega_{*}=\sqrt{c_{0} / m}$. С подобным эффектом мы уже сталкивались на примере кулачкового механизма с пружинным замыканием (см.п.2.2), когда оказалось, что при определенной частоте вращения $\omega=k_{\mathrm{i}}$ на кулачок действует лишь постоянная составляющая замыкающей силы; переменная же гармоническая составляющая уравновешивается силой инерции толкателя при его программном движении. Графики максимальных реакций $R_{\max }^{0}, R_{\max }$ при отсутствии разгружателя, (кривая 1) и после его установки (кривая 2) приведены на рис.3.13. При $\omega=\omega_{*}=\sqrt{c_{0} / m}$ имеем $R_{\max }^{0}=0$.

Подчеркнем, что $\omega_{*}$ не совпадает с собственной частотой $k=\sqrt{\left(c_{0}+c\right) / m}$, где $c$ - приведенный коэффициент жесткости механизма. Обычно $\omega_{*} \square k$, и лишь при отсоединении выходного звена от привода $(c \rightarrow 0)$ имеем $\omega_{*} \rightarrow k$. Точка пересечения обеих кривых определяет частотный интервал $\omega>\omega_{0}=\omega_{*} / \sqrt{2}$, на котором установка разгружателя дает положительный эффект. При пуске $R_{\max }(\omega)=c_{0} y_{0}=m \omega_{*}^{2} y_{0}$, поэтому установка разгружателя приводит к увеличению пускового момента. Это является недостатком, который 
может быть устранён при применении разгрузочного механизма с частотной настройкой (см. ниже).

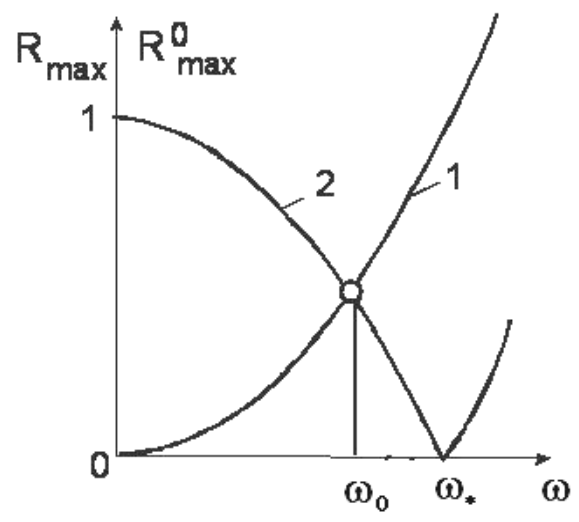

Рис. 3.13. К определению $\omega_{0}$

Независимо от конструктивных особенностей разгружатель, как правило, является аккумулятором энергии либо потенциальной, либо кинетической. В первом случае используются пружины или пневматические устройства; во втором - инерционные звенья, приводимые в движение специальными уравновешивающими механизмами, в качестве которых обычно используют рычажные и кулачковые механизмы с достаточно массивными выходными звеньями.

В разгружателях первого типа усилие противодействия является функцией положения и не зависит от скорости, в то время как уравновешиваемые динамические нагрузки пропорциональны квадрату угловой скорости входного звена. Поэтому такие устройства должны настраиваться на определенный скоростной режим.

В устройствах второго типа разгружающее усилие также пропорционально квадрату угловой скорости, а следовательно, по отношению к динамическим составляющим нагрузки оно оказывается следящим. В более совершенных разгружателях имеется программоноситель, роль которого выполняет какой-либо цикловой механизм, например кулачковый или рычажный.

Для повышения эффективности разгружателя и устранения возможности дополнительного возбуждения колебаний системы место его установки должно быть по возможности приближено к источнику колебаний. С этой точки зрения целесообразно его непосредственно присоединять к выходному звену основного механизма. На рис 3.14 , а, в показано несколько разновидностей разгружателей, которые состоят из простейшего рычажного механизма и пружины (на рис. 3.14, б, в сохранено лишь выходное звено основного механизма и сам разгружатель).

Для компенсации инерционных нагрузок выходного звена разгружатель должен аккумулировать энергию в период выбега и возвращать ее в период разбега. Это достигается сменой знака разгружающего момента при переходе от разбега к выбегу. 
a)

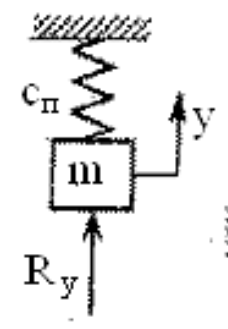

6)

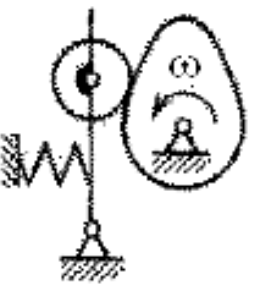

8)

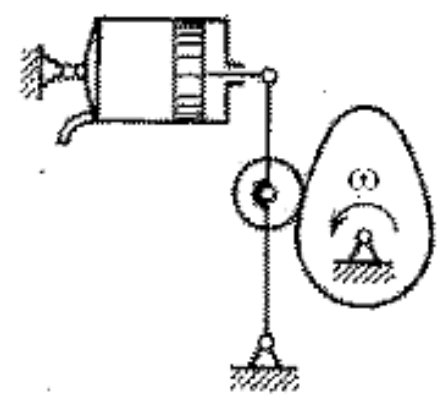

Рис.3.14. Схемы динамических разгружателей

a)

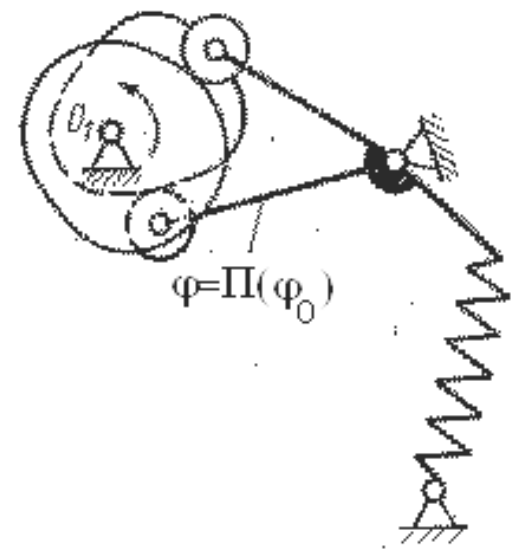

6)
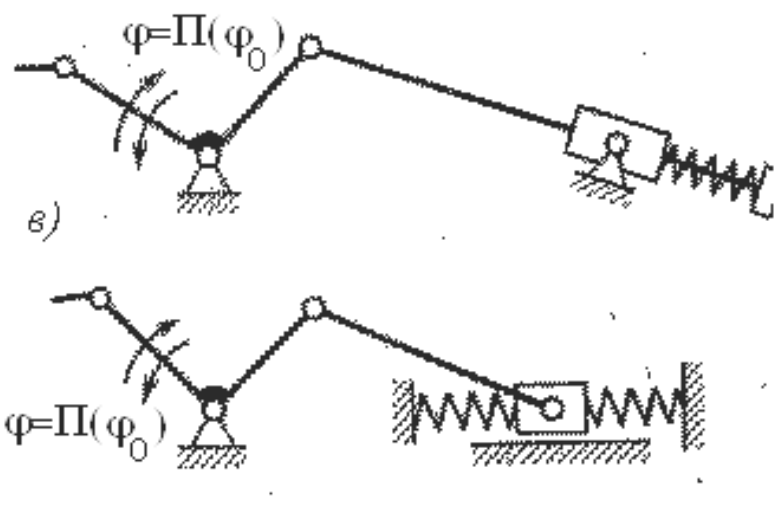

Рис.3.15. Пружинные динамические разгружатели

Методика расчета. В общем случае абсолютная величина реакции со стороны рабочего органа на привод машины равна

$$
|R|=\left|R_{*}+U\right|,
$$

где $R_{*}$ - реакция при отсутствии разгружателя; $U$ - дополнительная составляющая реакции за счет установки разгружателя.

Если воспользоваться пружинным разгружателем (см. рис.3.15,б), то

$$
U=u_{0}+u_{1} y,
$$

где $u_{0}$ - предварительная деформация пружины; $u_{1}=c_{0}-$ коэффициент жесткости пружины. Функция $R_{*}(y)$ в общем случае может не совпадать на прямом и обратном ходе. Следуя методу наименьших квадратов, определим параметры $u_{0}$ и $u_{1}$ таким образом, чтобы обеспечить минимум функционала

$$
\Psi=\int\left(R_{*}+U\right)^{2} d y \rightarrow \min
$$

Пусть $R_{*}(y)$ является двузначной функцией, а именно $\vec{R}_{*}(y)-$ на прямом ходе и $\bar{R}_{*}$ - на обратном. Тогда условию (3.30) отвечает 


$$
\frac{\partial \Psi}{\partial u_{i}}=2\left[\int_{0}^{y_{\max }}\left(\vec{R}_{*}+U\right) \frac{\partial U}{\partial u_{i}}|d y|+\int_{0}^{y_{\max }}\left(\bar{R}_{*}+U\right) \frac{\partial U}{\partial u_{i}}|d y|\right]=0 \quad(i=0,1) .
$$

Если $y=\Pi(\varphi)$, где $\varphi-$ угол поворота входного звена, то $|d y|=\left|\Pi^{\prime}(\varphi)\right| d \varphi$, где П'(ф) - первая геометрическая передаточная функция механизма. Принимая во внимание, что согласно (3.29) $\partial U / \partial u_{0}=1$ и $\partial U / \partial u_{1}=y$, на основании (3.31) получаем систему двух линейных алгебраических уравнений относительно $u_{0}$ и $c_{0}$

$$
\left.\begin{array}{l}
2 \Pi_{\max } u_{0}+\Pi_{\max } c_{0}=S_{1} \\
\Pi_{\max }^{2} u_{0}+\frac{2}{3} \Pi_{\max }^{3} c_{0}=S_{2}
\end{array}\right\}
$$

где $S_{1}=-\int_{0}^{2 \pi} R_{*}(\varphi)\left|\Pi^{\prime}(\varphi)\right| d \varphi ; \quad S_{2}=-\int_{0}^{2 \pi} R_{*}(\varphi) \Pi(\varphi)\left|\Pi^{\prime}(\varphi)\right| d \varphi$.

Если функция $R_{*}(\varphi)$ задана таблицей, графиком или имеет сложный аналитический вид, то для определения $S_{1}$ и $S_{2}$ может быть использован любой способ численного интегрирования. На основании (3.29) имеем

$$
u_{0}=\left(2 S_{1} \Pi_{\max }-3 S_{2}\right) / \Pi_{\max }^{2} ; \quad c_{0}=3\left(2 S_{2}-\Pi_{\max } S_{1}\right) / \Pi_{\max }^{3} .
$$

На рис. 3.16 в качестве иллюстрации показаны типичные графики $R_{*}(y)$, $U(y)$ и график результирующей реакции после установки разгружателя $R_{1}(y)=R_{*}(y)+U(y)$. Из графиков следует, что в данном примере максимальная реакция на выходном звене уменьшилось примерно в 4 раза.

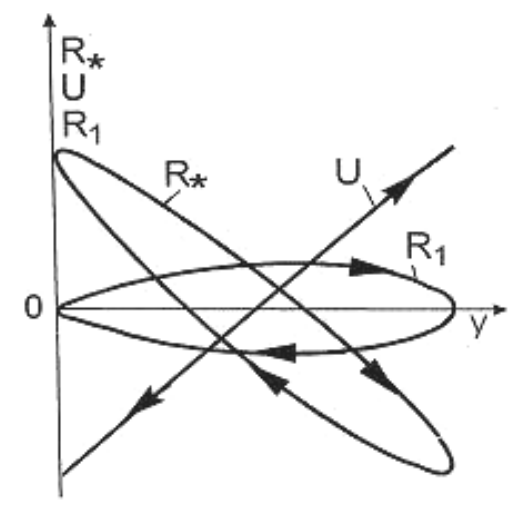

Рис. 3.16. К анализу эффективности динамической разгрузки

При гармоническом перемещении рабочего органа (см. выше) график динамической составляющей реакции $R_{*}(y)$ вырождается в прямую, а $U(y)$ - в её зеркальное отображение. При этом на расчетной частоте $\omega_{*}$ график $R_{1}(y)$ 
совпадает с осью абсцисс, т.е. имеет место абсолютная динамическая разгрузка.

При применении разгружающих устройств следует, однако, учитывать возможность существенного уменьшения реакций в кинематических парах, что может привести к возникновению виброударных режимов в зазорах (см.гл.4). Кроме того, при разгрузочных устройствах пружинного типа необходима проверка влияния колебаний пружин (см. ниже пп.4.4, 4.5).

Динамический разгружатель с зарезонансной частотной настройкой. Динамическая модель этого разгружателя приведена на рис.3.17, а.В отличие от РУ, показанного на рис.3.17, б, данный разгружатель представляет собой защемлённую с одной стороны рессору, на другом конце которой расположена дополнительная масса $m_{2}$, а в промежуточном сечении приведенная масса исполнительного органа $m_{1}[15]$.

При соответствующем выборе параметров и малых значениях ш форма колебаний упругих элементов не имеет узлов, поэтому приведенная к рабочему органу жесткость рессор относительно мала (рис.3.15,б). При выходе системы на рабочий скоростной режим формы колебаний рессор будут иметь узлы колебаний, что эквивалентно установке в этих сечениях дополнительных опор, создающих эффект увеличения приведенной жесткости до расчетного значения, необходимого для уравновешивания динамических нагрузок (рис.3,в; показанная на рисунке опора $\mathbf{O}$ носит условный характер).
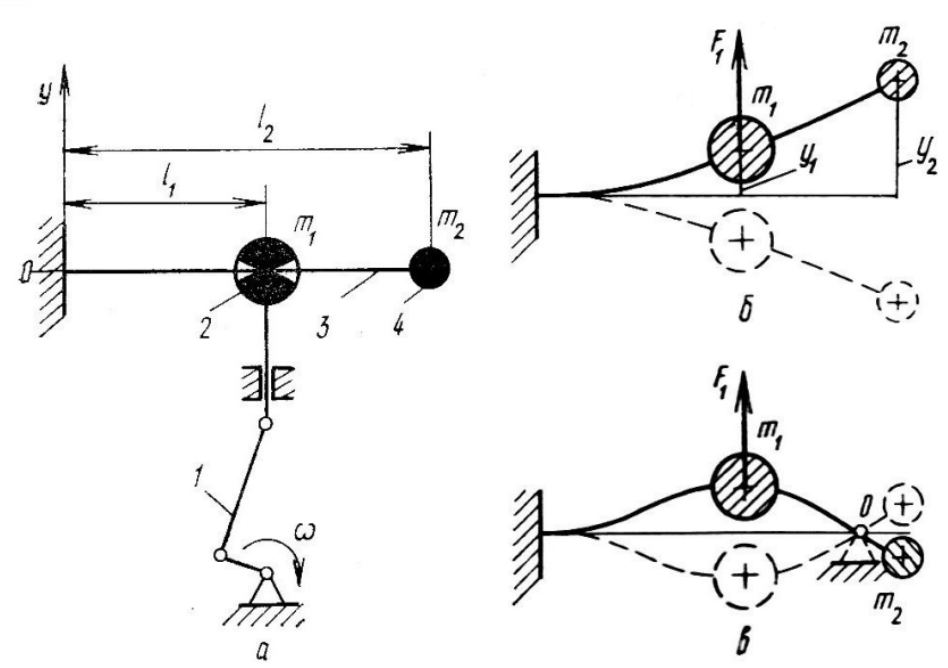

Рис. 3.17. Динамический разгружатель рессорного типа

В соответствии с принятой моделью колебания динамического разгружателя описываются системой уравнений

$$
\left.\begin{array}{l}
y_{1}=e_{11}\left(F_{1}-m_{1}\right)-e_{12}\left(m_{2}+R_{2}\right) ; \\
y_{2}=e_{21}\left(F_{1}-m_{1}\right)-e_{22}\left(m_{2}+R_{2}\right),
\end{array}\right\}
$$


где $y_{1}, y_{2}$ - перемещение масс $m_{1}, m_{2} ; e_{11}=l_{1}^{3} /(3 E I) ; e_{22}=l_{2}^{3} /(3 E I)$;

$e_{12}=e_{21}=l_{1}^{2}\left(l_{2}-l_{1} / 3\right) /(2 E I) ; E, I$ - модуль упругости и момент инерции сечения рессоры; $F_{1}$ - вертикальная составляющая реактивной силы, действующей на массу $m_{1}$ со стороны механизма; $R_{2}=b_{2} \&$ - линеаризированная сила сопротивления, приведенная к сечению массы $m_{2}$. Силы сопротивления не оказывают существенного влияния на частотную настройку разгружателя, что позволяет на этой стадии исключить их из рассмотрения. Исследуемый колебательный процесс, вызванный кинематическим возмущением $y_{1}(t)$, является заданной периодической функцией времени, которую представим в виде ряда Фурье

$$
y_{1}(t)=\sum_{j=1}^{\infty} a_{1} \sin \left(j \omega t+\vartheta_{j}\right)
$$

где $a_{1 j}=\sqrt{a_{j c}^{2}+a_{j s}^{2}} ; a_{j c}, a_{j s}$-коэффициенты Фурье;

$\vartheta_{j}=\operatorname{arctg}\left(a_{j c} / a_{j c}\right) ; \omega=2 \pi / T-$ частота вращения входного звена; $T$ - период кинематического цикла; $j$ - номер гармоники.

Принимая

$$
y_{2}(t)=\sum_{j=1}^{\infty} a_{2 j} \sin \left(j \omega t+\vartheta_{j}\right) ; \quad F_{1}(t)=\sum_{j=1}^{\infty} F_{1 j}^{*} \sin \left(j \omega t+\vartheta_{j}\right),
$$

после подстановки (3.35), (3.36) в (3.34) и элементарных преобразований находим выражение для амплитуды $j$-й гармоники силы $F_{1}$ :

$$
F_{1 j}^{*}=a_{1} \frac{\mu m_{1}^{2} e_{11}^{2} \varepsilon_{*} j^{4} \omega^{4}-m_{1} e_{11}\left(1+\mu \varepsilon^{3}\right) j^{2} \omega^{2}+1}{e_{11}\left(1-\mu m_{1} e_{11} \varepsilon_{*} j^{2} \omega^{2}\right)},
$$

где $\mu=m_{2} / m_{1} ; \quad \varepsilon=l_{2} / l_{1} ; \quad \varepsilon_{*}=\varepsilon^{3}-0,25(3 \varepsilon-1)$.

Функция $F_{1 j}^{*}$ для гармоники $j=v$ обращается в нуль два раза: при $\omega=\omega_{1}, \omega=\omega_{2}$, где $\omega_{1}, \omega_{2}-$ корни биквадратного уравнения, полученного при обращении числителя (3.37) в нуль. При этом

$$
\omega_{1,2}=\frac{1}{v} \sqrt{\frac{1+\mu \varepsilon^{3} m \sqrt{\left(1+\mu \varepsilon^{3}\right)^{2}-4 \mu \varepsilon_{*}}}{2 \mu m_{1} e_{11} \varepsilon_{*}}}
$$

Полученные значения $\omega_{1}, \omega_{2}$ при $v=l$ соответствуют собственным частотам „разомкнутой" двухмассовой системы (при отсоединении рессоры от передаточного механизма). На этих частотах кинематического возмущения происходит полное уравновешивание заданной гармоники $j=v$ динамической нагрузки $F_{1}$. В качестве частоты настройки, соответствующей 
расчетному рабочему режиму, целесообразно принять зарезонансную частоту $\omega_{2}$. Резонансная частота, пересекаемая разгружателем при выходе на расчетный режим, соответствует собственной частоте разгружателя при абсолютно жёсткой связи рессоры с передаточным механизмом и определяется из условия равенства нулю знаменателя выражения (3.37)

$$
\omega_{p}=v^{-1} \sqrt{3 E I /\left(\mu m_{1} \varepsilon_{*} l_{1}^{3}\right)}
$$

При переходе через резонансную зону ограничение амплитуд $F_{1 j}^{*}, a_{2 j}$ достигается за счёт конструкционного демпфирования. Учет в (3.31) ранее опущенных диссипативных членов $R_{2}$ позволяет получить выражение для определения реактивного усилия привода вблизи резонанса и вне зон оптимальных динамических нагрузок

$$
F_{1}(t)=\sum_{j=1}^{\infty} F_{1 j}^{*} \sin \left(j \omega t+\vartheta_{j}+\gamma_{j}\right)
$$

где

$F_{1 j}^{*}=a_{1 j} e_{11}^{-1} A_{j} ; \quad A_{j}=\sqrt{B_{j}^{2}+2 B_{j} C_{j} \cos \alpha_{j}+C_{j}^{2}} ; \quad B_{j}=1-e_{11} m_{1} j^{2} \omega^{2} ;$

$C_{j}=0,5(3 \varepsilon-1) e_{11} \mu m_{1} j \omega \kappa_{j} D_{j} ; \quad \kappa_{j}=0,5(3 \varepsilon-1) / \sqrt{\left(1-j^{2} z^{2}\right)^{2}+4 \delta^{2} j^{2} z^{2}} \quad$-коэффициент динамичности; $z=\omega / k$ - коэффициент частотной расстройки; $\delta=\psi /(4 \pi) ; \psi-$ коэффициент рассеяния;

$$
\begin{aligned}
& D_{j}=\sqrt{4 \delta^{2}+j^{2} \omega^{2}} ; \quad \alpha=\beta_{j}-\Delta_{j} ; \quad \beta_{j}=\operatorname{arctg}\left[b_{2} /\left(\mu m_{1} j \omega\right)\right] ; k=\sqrt{e_{11} /\left[\mu m_{1}\left(e_{11} e_{22}-e_{12}^{2}\right)\right]} ; \\
& \Delta_{j}=\operatorname{arctg}\left[2 j z \delta /\left(1-j^{2} z^{2}\right)\right] ; \quad \gamma_{j}=\operatorname{arctg}\left[C_{j} \sin \alpha_{j} /\left(\beta_{j}+C_{j} \cos \alpha_{j}\right)\right] .
\end{aligned}
$$

Методику синтеза динамического разгружателя проиллюстрируем на примере привода рабочего органа, снабженного аксиальным кривошипноползунным механизмом (рис. 3.17,a ) при следующих исходных данных: радиус кривошипа $r=9,2 \mathrm{мм}$, длина шатуна $l=100 \mathrm{мм}$, приведенная масса $m_{1}=3,13$ кг, $l_{1}=120 \mathrm{мм}, \omega_{2}=120 c^{-1}, \mu=0,2, \varepsilon=0, h=2$ мм, $b=26,4$ мм, где $h-$ толщина, а $b-$ ширина сечения рессоры.

Анализ графиков динамических нагрузок, представленных на рис. 3.18, показывает, что РУ обеспечивает практически полное уравновешивание первой гармоники нагрузки на заданной частоте настройки $\omega_{2}=120 c^{-1}$. Оценить эффективность уравновешивания можно с помощью коэффициента разгрузки $\zeta(\omega)=F^{*} / F_{1}^{*}$, показывающего, во сколько раз снижается амплитудное значение реактивного усилия $F_{1}^{*}$ привода с РУ в сравнении с аналогичным усилием $F^{*}$ привода без РУ (кривая 1). 


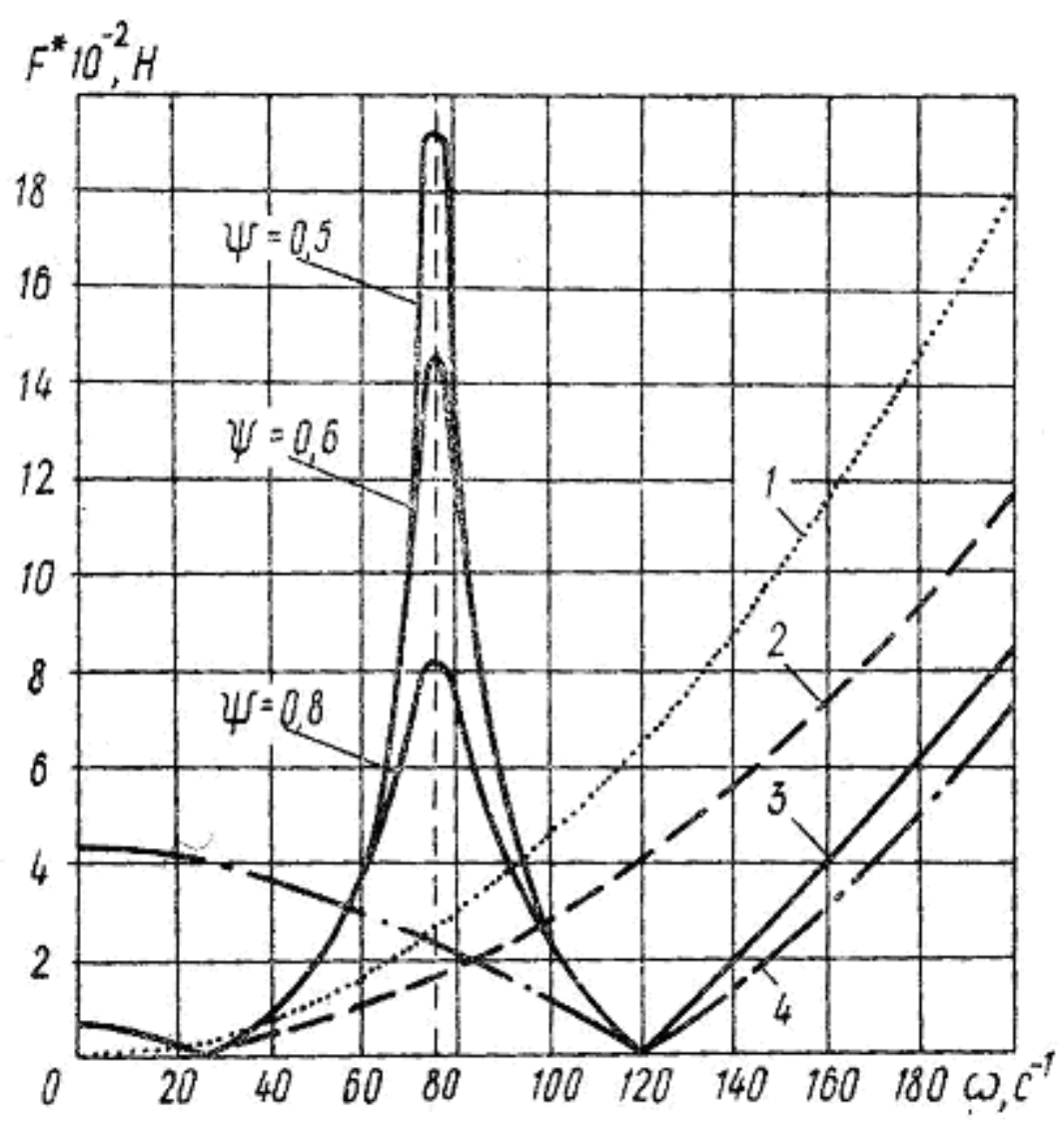

Рис. 3.18. К анализу эффективности динамической разгрузки

В нашем случае на частоте настройки при значениях коэффициента рассеяния энергии $\psi=0,5-0,8$ имеем $\zeta=2,78-2,56$. Кривые 2,4 соответствуют пружинному разгружателю, кривая 3 - рессорному разгружателю с зарезонансной настройкой.

На графиках также видно (кривые 3, 4), что в сравнении с РУ пружинного типа пусковая нагрузка на привод снижается более чем в 7 раз. Для обеспечения стабильной работы динамического разгружателя значение частоты настройки $\omega_{2}$ должно быть удалено из резонансной зоны. Выполнение этого условия обеспечивается соответствующим выбором параметров $\varepsilon, \mu, I$. Выявлено, что с учетом конструктивных ограничений наиболее рационально принять $\varepsilon=2$ и $\mu=0,2$.

Отличительной особенностью данного разгружателя также является возможность осуществления эффективной настройки на другую рабочую частоту $\omega_{2}$ без обязательной для пружинного разгружателя замены упругих элементов ( $I$ = const). Такая настройка легко выполняется путем изменения массы дополнительного груза и его положения на рессоре. При увеличении значений $\varepsilon$ и $\mu$ частота настройки $\omega_{2}$ снижается, а при их уменьшении - увеличивается, что позволяет обеспечить полное уравновешивание заданной гармоники динамической нагрузки на разных скоростных режимах, причём без демонтажных работ.

Для проверки достоверности полученных результатов и эффективности разгружателя было проведено экспериментальное исследование на специальной 
стендовой установке ${ }^{1}$. На рис. 3.19 представлены графики амплитуд динамических нагрузок, полученные теоретическим и экспериментальным путем. При этом пунктиром и сплошной кривой отображены теоретические результаты $F^{*}$ и $F_{1}^{*}$ соответственно без ДР и при использовании ДР. Экспериментальным результатам отвечают точки, показанные треугольниками и кружочками. Анализ показывает, что на частоте настройки $\omega_{2}=28,1 \mathrm{c}^{-1}$ амплитуда динамической нагрузки $F^{*}=50,60 \pm 0,85 \mathrm{H}$ после установки снижается до $F_{1}^{*}=21,28 \pm 0,19(\zeta=2,38)$, что эквивалентно работе привода без ДР при $\omega_{2}=18,3 \mathrm{c}^{-1}$.

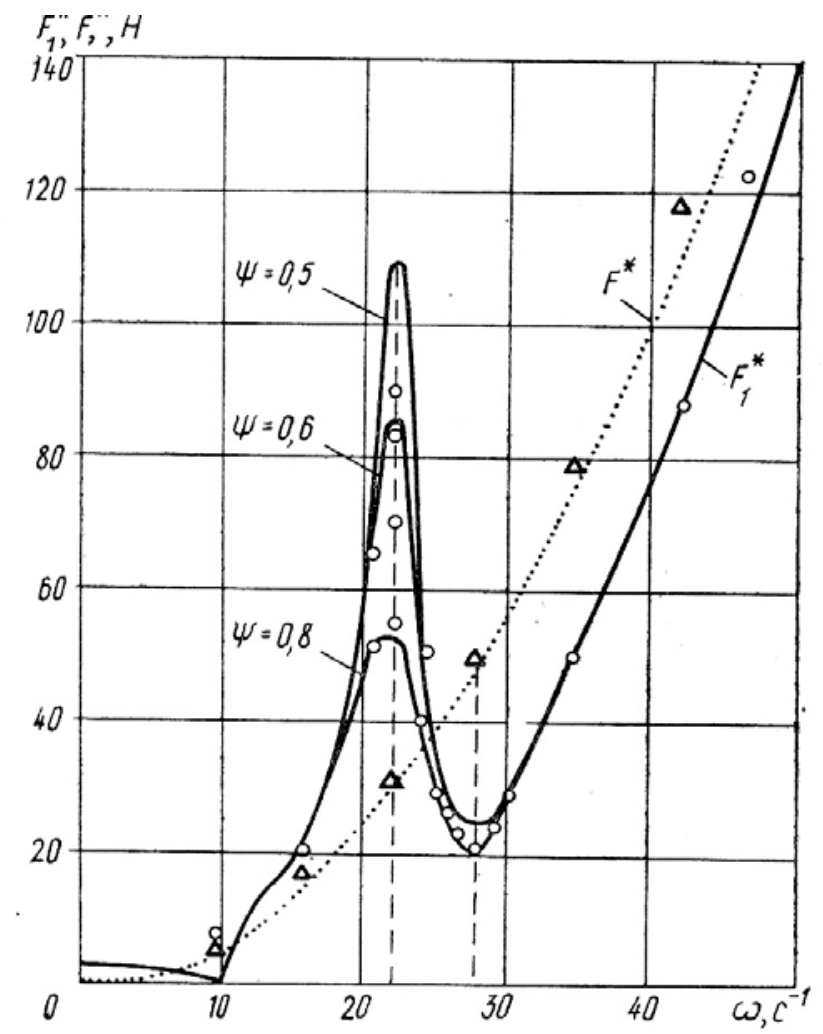

Рис. 3.19. Сопоставление теоретических и экспериментальных результатов

Полученные в зоне разгрузки экспериментальные результаты хорошо согласуются с теоретическими. Так, при $\omega=\omega_{2} ; F^{*}=49,61 \mathrm{H}, F_{1}^{*}=20,66 \mathrm{H}, \zeta=2,41$ отклонение экспериментальных нагрузок от теоретических в зоне $\omega_{2}$ не превышает 3\%.

Анализ показывает, что в зоне $\omega>\omega_{2}$ всегда обеспечивается частичное уравновешивание. Так, например, при $\omega=34,7 \mathrm{c}^{-1}$ имеем $\zeta=1,57$. Из графика видно, что динамическая нагрузка в резонансной зоне возрастает, однако при использовании демпфирования она может быть существенно снижена. Экспериментальные исследования показали, что при обеспечении быстрого пуска и выхода на расчетный режим резонанс не успевает развиться, но необходимо

\footnotetext{
1 Экспериментальное исслеование проведено А.П. Тышкуном.
} 
обеспечить достаточно интенсивное торможение при остановке машины. Следует однако иметь в виду, что чрезмерно сильное демпфирование снижает эффект разгрузки.

По сравнению с ДР пружинного типа использование разгружателя с зарезонансной частотной настройкой позволяет в период пуска более чем в 7 раз снизить нагрузку на входные звенья передаточного механизма и привод. 


\section{ГЛАВА 4. ВЛИЯНИЕ ЗАЗОРОВ НА ИСКАЖЕНИЯ ЗАКОНОВ ДВИЖЕНИЯ}

\section{1. Динамические эфрфекты}

Зазор, как правило, является сопутствующим фактором любой кинематической пары, осуществляющей подвижное соединение звеньев механизма. При отсутствии зазоров даже при идеальном изготовлении элементов кинематической пары возникает опасность существенного роста реакций. В частности, это наблюдается при температурном расширении деталей, что, в свою очередь, вызывает увеличение сил трения и, в конечном итоге может даже привести к полному заклиниванию механизма. Проблема рационального динамического синтеза колебательных систем с учетом зазоров особо остро встает при проектировании высокоскоростных цикловых механизмов с нелинейной функцией положения, поскольку в этом классе механизмов зазоры могут привести к большим искажениям заданного программного движения рабочих органов, а также к повышению шума и виброактивности привода. Нередко именно величина зазоров в конечном итоге лимитирует производительность и эксплуатационные характеристики многих современных технологических машин.

На рис.4.1 показаны экспериментальные записи ускорений, полученные при динамическом анализе двух массивных рабочих органов трикотажной машины, каждый из которых приводится в движение тремя цикловыми механизмами, работающими в параллельной схеме.

a)

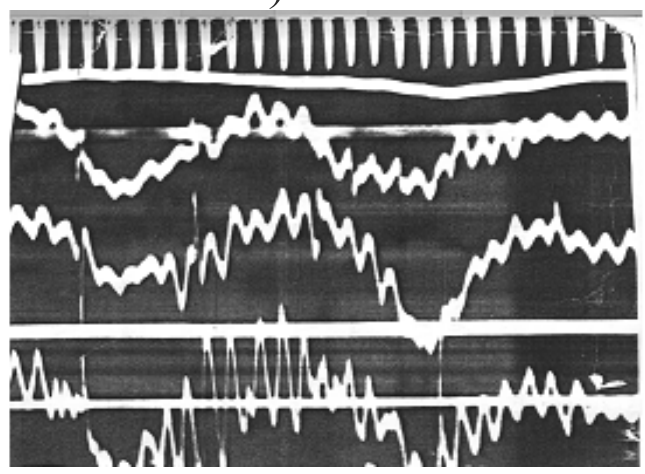

б)

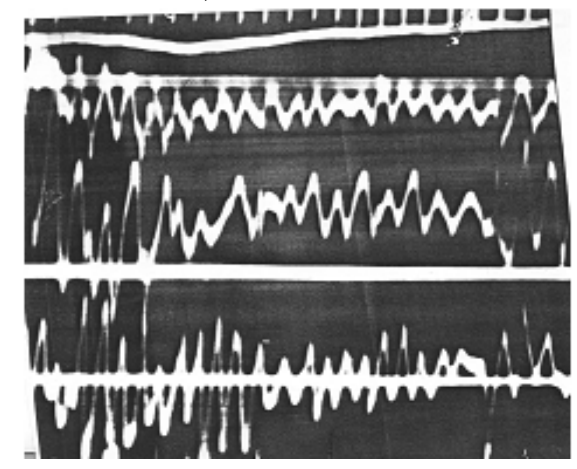

Рис.4.1. К анализу влияния зазоров на кинематические характеристики

Если на первой осциллограмме (рис.4.1, а) еще можно распознать заложенный исходный закон изменения ускорений, то на второй (рис.4.1, б) - он полностью перекрыт колебаниями.

По воздействию на колебательную систему можно условно выделить два характерных случая проявления зазора, каждому из которых отвечает соответствующая область изменения параметров и внешних возмущений. В nеpвом случае зазор проявляет себя как нелинейный элемент, существенно влияющий на спектр частот свободных колебаний. Во втором случае влияние за- 
зора в основном проявляется как импульсное возмущение на ограниченном временном интервале кинематического цикла при отсутствии сколько-нибудь заметного искажения частотного спектра исходной линейной системы. При этом однако сохраняется возможность многократных соударений, приводящих к виброударным режимам, при которых динамический эффект от зазоров приближается к первому случаю. Как показывает анализ, при практически реализуемых значениях параметров переход из одной фазы в другую и относительно малом отрезке времени этого перехода происходит так называемый квазипластический удар. При этом возникает быстро затухающий высокочастотный дребезг. По существу, наблюдаемый при этом эффект близок абсолютно неупругому удару.

Проблема рационального динамического синтеза колебательных систем с учетом зазоров особо остро встает при проектировании высокоскоростных цикловых механизмов с нелинейной функцией положения, поскольку в этом классе механизмов зазоры могут привести к большим искажениям заданного программного движения рабочих органов, а также к повышению шума и виброактивности привода. Нередко именно величина зазоров в конечном итоге лимитирует производительность и эксплуатационные характеристики многих современных технологических машин, что заставляет существенно ужесточить точностные требования при их изготовлении и сборке.

Восстанавливающая сила, соответствующая схеме (рис.5.2, а) и графику $F(q)$ (рис.4.2, б), описывается следующим образом:

$$
F=c(q-\Delta \operatorname{sign} q) \eta(|q|-\Delta)
$$

где $q$ - обобщенная координата; $\Delta=0,5 s ; s$ - величина зазора; $\eta$ - единичная функция ( $u=0$ при $|q|<\Delta ; \eta=1$ при $|q| \geq \Delta)$.

Скачкообразное изменение единичной функции соответствует моменту перестройки структуры колебательной системы. С этими моментами переключения иногда связаны определенные трудности при их реализации.

a)

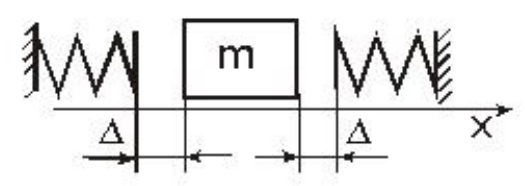

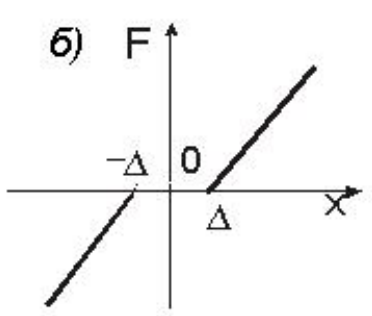

Рис. 4.2. Динамическая модель упругого элемента с зазором

Так, например, при использовании численных методов могут появиться особые «скользящие» режимы с большим числом переключений на ограниченном интервале времени. При аналитических исследованиях ступенчатый характер зависимостей может осложнить анализ и оптимизационный синтез из-за нарушения условий дифференцируемости в окрестности указанных зон. 
Для исключения этого недостатка при сохранении нелинейных свойств функции $F(q)$ используют аппроксимацию ступенчатых функций непрерывными функциями

$$
u(x)=\lim _{L_{0} \rightarrow \infty}\left[0,5+\pi^{-1} \operatorname{arctg}\left(\mathrm{L}_{0} x / \Delta\right)\right]_{*} \approx 0,5+\pi^{-1} \operatorname{arctg}(\operatorname{L} x / \Delta),
$$

где $x=|q|-\Delta ; L \square 1$ (обычно число $L$ должно на несколько порядков превышать $x / \Delta)$.

При смене знака $x$ функция $u(x)$ согласно (4.2) достаточно быстро изменяется от 0 до 1, сохраняя при этом непрерывный характер. Подобную процедуру иногда называют гладкой аппроксимацией. Как показывает эксперимент, гладкая аппроксимация зазора нередко оказывается даже более точной, чем исходная ступенчатая функция, что, по-видимому, связано с уменьшением контактной жесткости элементов кинематической пары в окрестности моментов переключений, наличием слоя смазки и другими сглаживающими факторами.

\section{2. Возбуждение колебаний при ударном характере взаимодействия элементов кинематических пар}

Особенности проявления зазоров при колебаниях проиллюстрируем на примере циклового механизма, динамическая модель которого приведена на рис.4.3.

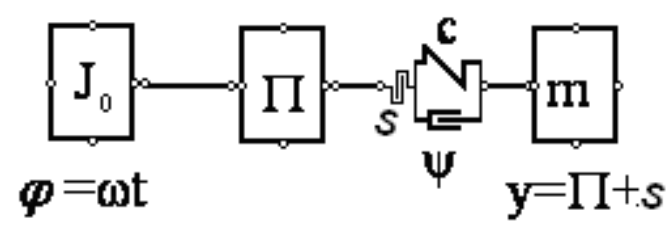

Рис.4.3. Динамическая модель циклового механизма с зазором

Помимо традиционных элементов на модели показан элемент $s$, отвечающий зазору. Этой модели при учете (5.1), (5.2) соответствует следующее дифференциальное уравнение:

$$
\ddot{q}+k_{0}^{2}\left(2 \delta k_{0}^{-1} \dot{q}+q\right) \Psi(q)=w(t) .
$$

Здесь принято, что абсолютная координата выходного звена представле-

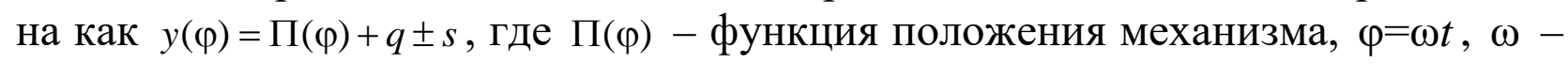
угловая скорость входного звена, $q-$ обобщенная координата; $k_{0}=\sqrt{c / m}-$ собственная частота при отсутствии зазора; $\Psi(q)=F(q) / m$ (см. формулу (4.1)).

Пусть $w(t)=w_{0} \cos \omega t$, где $w_{0}=\Pi_{\max }^{\prime \prime} \omega^{2}$, что отвечает гармоническому закону движения выходного звена без учета колебаний. В частности, такая функция положения с достаточной точностью отвечает перемещению ползуна кривошипно-ползунного механизма $\Pi \approx r_{0}(1-\cos \varphi)$. Для того чтобы придать ре- 
зультатам анализа более общий вид, перейдем к новой переменной, в качестве которой примем «безразмерное время» $\varphi=\omega t$. Тогда уравнение (4.3) примет вид

$$
\bar{q}^{\prime \prime}+N^{2}\left(2 \delta N^{-1} \bar{q}+\bar{q}\right) \Psi(\bar{q})=\cos \varphi,
$$

где $\quad N=k_{0} / \omega, \delta \approx \psi /(4 \pi) ;$ штрих отвечает производной по $\varphi ; \bar{q}=q / r_{0}-$ безразмерная координата.

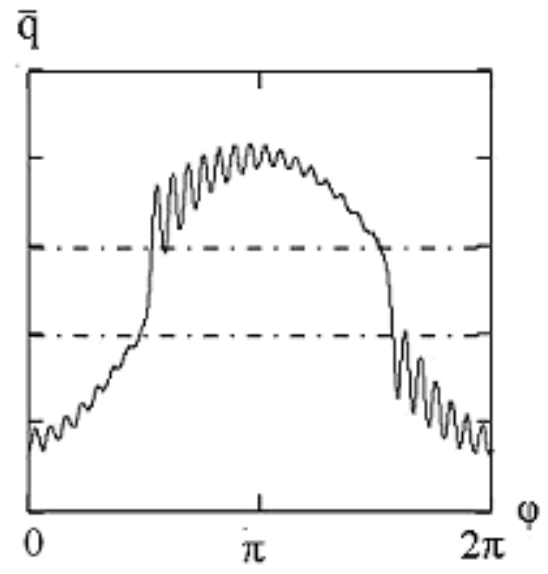

Рис.4.4. Графики $\bar{q}\left(\varphi_{1}\right)$

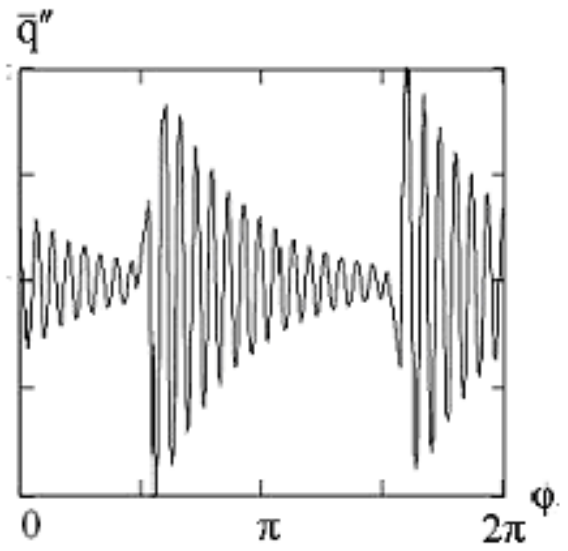

Рис.4.5. Графики $\bar{q}^{\prime \prime}\left(\varphi_{1}\right)$

На рис. 4.4 приведен график $\bar{q}\left(\varphi_{1}\right)$, полученный решением уравнения (4.4) численным методом при $N=30, \delta=0,03, \bar{s}=s / r_{0}=10^{-3}$. Перекладка в зазоре происходит при $-5 \cdot 10^{-4}<\bar{q}<5 \cdot 10^{-4}$ (границы этого интервала показаны на графике штрих пунктирными линиями). На графике четко видно импульсное возбуждение колебаний после каждого перехода через зазор. Поскольку на рассматриваемом режиме после выборки зазора отсутствуют повторные соударения, зазор практически не влияет на спектр собственных частот. Еще более наглядно это видно на графике функции $\vec{q}^{\prime \prime}\left(\varphi_{1}\right)$, которой пропорциональны силы инерции, возникающие при колебаниях (рис.4.5).

Совсем иная картина наблюдается при возникновении виброударных режимов. В качестве примера на рис.4.6 показан график $\bar{q}\left(\varphi_{1}\right)$ при сохранении принятых выше исходных данных, но при полной динамической разгрузке и одиночном импульсном возбуждении. В данном режиме частота колебаний $k$ существенно ниже значения $k_{0}$.

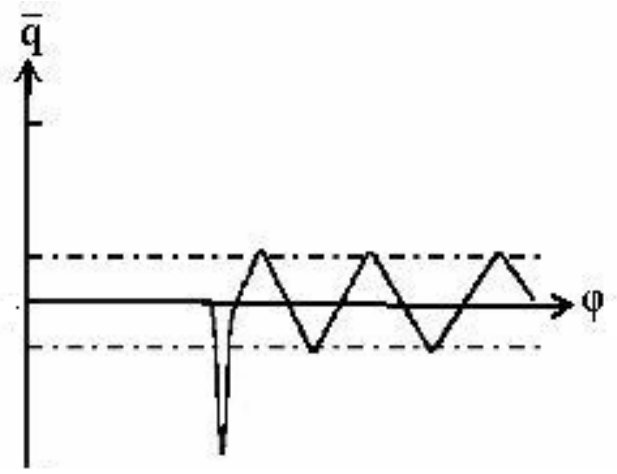

Рис. 4.6. Виброударный режим при повторных соударениях в зазоре 
Возможность возникновения подобных виброударных режимов должна быть исключена или ограничена еще на стадии создания машины. В связи с рассмотренным режимом при наличии зазоров также представляет интерес отрицательный эффект от полной динамической разгрузки (см. ниже).

a)

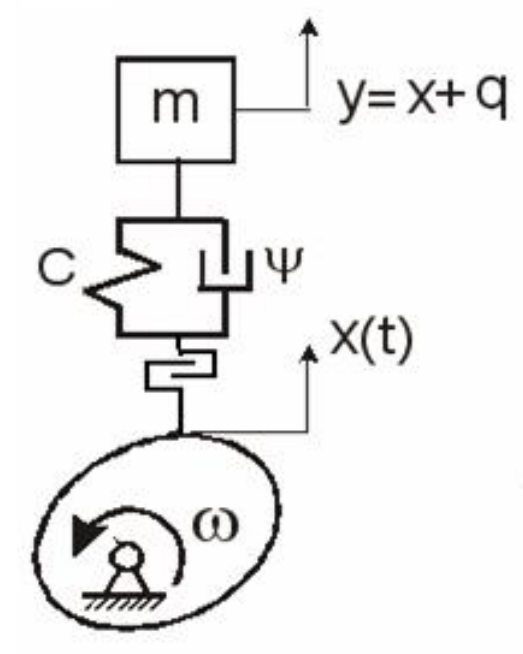

6)

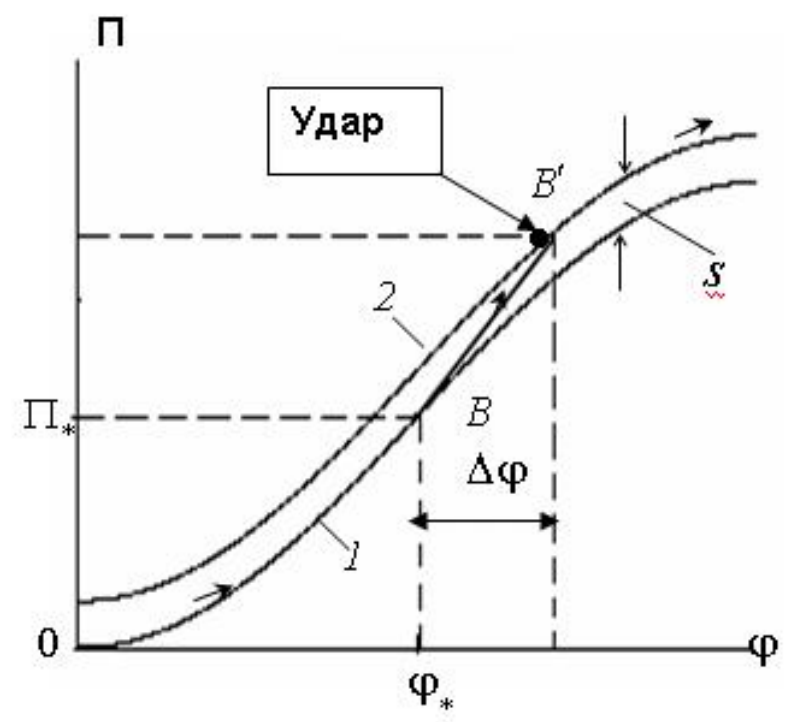

Рис.4.7. К определению динамического эффекта от перехода через зазор

Далее приведём некоторые аналитические оценки и динамические критерии. Динамический эффект от перекладки в зазоре проанализируем на примере кулачкового механизма (рис.4.7, $a$ ), реализующего функцию положения толкателя П(ф) (рис.4.7, б). Проведем две кривые на графике функции положения П(甲), смещенные на приведенную величину зазора $s$ (рис.4.7, б, кривые $1,2)$ ). Пусть при $\varphi=\varphi_{*}$ в точке $B$ происходит отрыв от кривой 1 , а в точке $B^{\prime}$ на кривой 2 происходит восстановление кинематического контакта. Примем, что угол поворота $\Delta \varphi=\omega \Delta t$, соответствующий зоне «свободного полета», мал. При этом с достаточным основанием можно считать, что движение на этом участке происходит с постоянной скоростью, равной скорости отрыва в точке $B$.

Согласно дифференциальному уравнению (4.3) будем различать две фазы движения. На первой фазе зазор выбран, следовательно, с точностью до постоянной $y=\Pi(\varphi)+q$. На второй фазе- при переходе через малый зазор, выходное звено перемещается по инерции практически с постоянной скоростью. Определим уровень дополнительных колебаний, возбуждаемых при восстановлении кинематического контакта. Тогда

$$
\Pi\left(\varphi^{*}+\Delta \varphi_{1}\right)+s=\Pi\left(\varphi^{*}\right)+\Pi^{\prime}\left(\varphi^{*}\right) \Delta \varphi,
$$

где $\varphi^{*}$ - угол поворота входного звена при смене знака реакции в кинематической паре; $s$ - величина зазора. 
Далее, представляя функцию П $\left(\varphi^{*}+\Delta \varphi\right)$ в окрестности $\varphi_{1}^{*}$ в виде усеченного ряда Тейлора, на основании (4.6) имеем

$$
\frac{1}{6} \Pi_{*}^{\prime \prime \prime} \Delta \varphi^{3}+\frac{1}{2} \Pi_{*}^{\prime \prime} \Delta \varphi^{*}+s=0 .
$$

Здесь $\Pi_{*}^{\prime \prime}, \Pi_{*}^{\prime \prime \prime}$ отвечают аргументу $\varphi^{*}$.

В современных высокоскоростных цикловых механизмах динамическая нагрузка, вызванная кинематическим возбуждением, обычно значительно превосходит силы сопротивления, поэтому переход через зазор практически происходит при $\Pi_{*}^{\prime \prime}=0$. Тогда согласно (4.6)

$$
\Delta \varphi_{1}=\sqrt[3]{6 s / \mid \Pi_{*}^{\prime \prime \prime}} .
$$

Здесь принято во внимание, что П"'< $<0$.

При восстановлении кинематического контакта имеет место скачок первой передаточной функции $\Delta \Pi^{\prime}$, который при учете (4.7) определяется следующим образом:

$$
\Delta \Pi^{\prime}=\sqrt[3]{4,5 s^{2}\left|\Pi^{\prime \prime \prime}\right|}
$$

Как было показано выше, при этом имеет место жесткий удар. Аналогичным образом могут быть учтены возмущения, связанные со скачками $\Delta \Pi "$ и $\Delta \Pi^{\prime \prime \prime}$. Введем в рассмотрение параметр $\xi=\left|\Delta y^{\prime \prime}\right|_{\max } /\left|\Pi^{\prime \prime}\right|_{\max }$, равный отношению экстремума дополнительного ускорения (после выборки зазора) к экстремуму идеального ускорения. При одиночном ударе получаем

$$
\xi=\Pi_{*}^{\prime \prime \prime} \sqrt{(4,5)^{2 / 3} \beta_{1}^{4} N^{2}+N^{-2}},
$$

где $\beta_{1}=\sqrt[3]{\mathrm{s} /\left|\Pi_{*}^{\prime \prime \prime}\right|}, \quad N=k_{0} / \omega$.

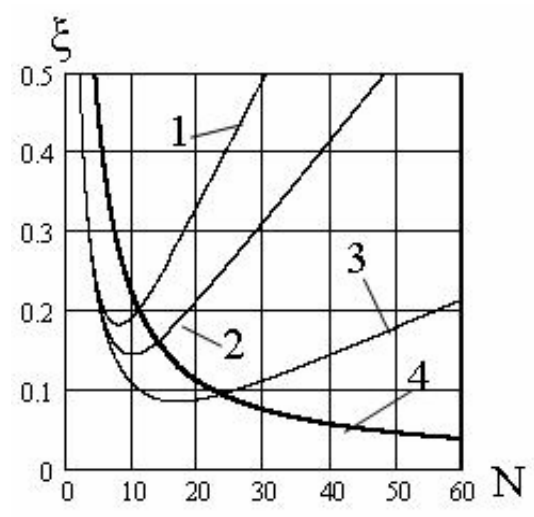

Рис. 4.8. Определение дополнительных ускорений при одиночном пересечении зоны зазора

На рис.4.8 представлено семейство кривых $\xi\left(N, \beta_{1}\right)$ при $\beta_{1}=10^{-3}$ (кривая 1), $\beta_{1}=5 \cdot 10^{-4}$ (кривая 2), $\beta_{1}=10^{-4}$ (кривая 3). Параметр $\beta_{1}$ играет роль важного динамического критерия. На основании формулы (4.9) могут быть определены параметры системы, удовлетворяющие требованию $\xi \leq \xi_{*}$, где $\xi_{*}-$ допускае- 
мое значение уровня дополнительных ускорений, вызванных зазором. (Обычно $\left.\xi_{*}<0,1 \div 0,2\right)$.

Для исключения возможности возникновения виброударных режимов при выборке зазора очень важно устранение повторных соударений. Повторные соударения могут возникнуть с некоторым фазовым сдвигом относительно угла $\varphi_{1}^{*}$, соответствующего разрыву кинематической связи при «входе»в зазор: $\Delta \varphi=\Delta \varphi_{1}+\Delta \varphi_{2}$ (см. рис. 4.4). Значение $\Delta \varphi_{1}$ определяется формулой (4.7), а $\Delta \varphi_{2} \approx \Delta t_{2} / \omega=\alpha N^{-1}$, где интервал времени $\Delta t_{2}$ соответствует первому минимуму функции $\bar{q}$ после выборки зазора, $\alpha \approx 4,45 \div 6,28$.

На основании (4.7)-(4.9) можно показать, что в первом приближении должно удовлетворяться условие

$$
\beta_{2}=\beta_{1} N^{-1}<0,3 \exp (\delta \alpha)[1+\sqrt{1+29,4 \exp (-\delta \alpha)}] .
$$

В формуле (4.10) учтено демпфирование колебаний на малом отрезке времени $\Delta t_{2}$. Однако, как показывает анализ, $\exp (\delta \alpha) \approx 1$. Тогда $\beta_{2}<\beta_{2}^{*}$, где $\beta_{2}^{*} \approx 1,9$. На рис.4.8 этому условию отвечают точки, расположенные ниже кривой 4.

\section{3. Разрывы кинематической цепи в механизмах с силовым замыканием и их устранение}

В современных машинах широко используются механизмы с неудерживающими связями, в которых для предотвращения разрывов контакта в кинематических парах применяется силовое замыкание, реализуемое с помощью пружин. С пружинным замыканием чаще всего приходится сталкиваться в кулачковых механизмах, однако нередко замыкающие пружины устанавливаются и на звеньях рычажных, кулачково-рычажных и других механизмов в целях частичного или полного устранения пересопряжений рабочих поверхностей кинематических пар, возникающих при перекладе в зазоре.

Надежное функционирование системы замыкания может быть нарушено по разным причинам, среди которых определяющий характер носят колебания самих пружин, а также колебания звеньев механизма, возбуждаемые в числе прочих и замыкающей силой. Если динамические нагрузки оказываются преобладающими, должно соблюдаться условие $F=\xi\left|P^{i}\right|_{\max }$, где $P^{i}-$ сила инерции, $F$ - замыкающая сила, $\xi>1$, коэффициент запаса. При колебаниях могут существенно изменяться как замыкающая сила, так и силы инерции, определённые на базе кинетостатической модели.

Последнее обстоятельство может привести к нарушению силового замыкания, не устраняемого повышением замыкающего усилия. Этот эффект связан с нестационарностью кинематической связи, из-за чего при нелинейной функции положения даже постоянная составляющая замыкающей силы трансформируется в переменный момент $M_{0}=\Pi^{\prime} F_{0}$, воздействующий на привод. При этом дополнительно возбуждаются вынужденные колебания вход- 
ного звена, которые вызывают приращение инерционных нагрузок на выходном звене. На рис.5.9 приведены две осциллограммы, полученные при записи ускорений толкателя на экспериментальной установке кулачкового механизма с пружинным замыканием [6].

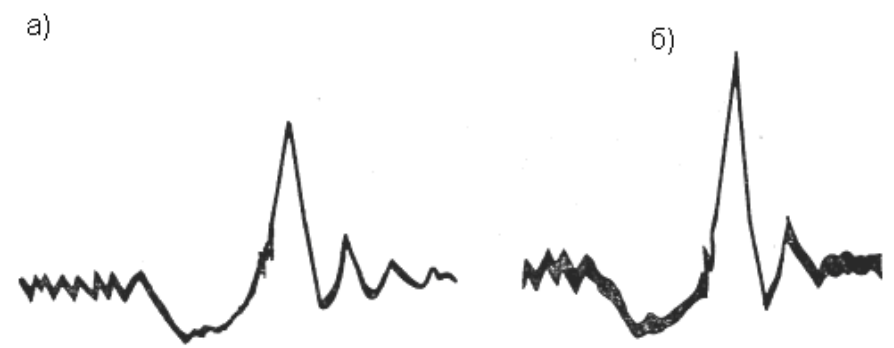

Рис. 4.9. Динамический эффект от увеличения замыкающей силы

Исходные данные при записи этих осциллограмм были идентичными, за исключением усилия предварительной деформации, которое во втором случае (рис.4.9,б) по сравнению с первым (рис.4.9,a) увеличено в 1,5 раза. В результате максимальное ускорение толкателя возросло примерно на 40\%.

Пусть $F=F_{0}+c_{0} \Pi$, где $c_{0}, F_{0}-$ коэффициент жёсткости и сила от предварительной деформации замыкающей пружины, П - функция положения выходного звена.

Между $\left|P^{i}\right|_{\max }$ и $F_{0}$ имеется функциональная связь $\left|P^{i}\right|_{\max }=\Psi\left(F_{0}\right)$. Дадим некоторое приращение силе $F_{0}$, равное $\Delta F_{0}$. Тогда, ограничиваясь линейным приближением, имеем

$$
\left|P^{i}\right|_{\max }+\Delta P^{i} \approx \Psi\left(F_{0}\right)+\frac{\partial \Psi}{\partial F_{0}}\left(F_{0}\right) \Delta F
$$

Введём понятие идеального приращения коэффициента запаса $\Delta \xi_{0}=\Delta F /\left|P^{i}\right|_{\max }$ при абсолютно жёстком приводе, т. е. при $\Delta P^{i}=0$. Используя (4.12), можно показать, что при учёте упругости привода $\Delta \xi=\zeta \Delta \xi_{0}$, где

$$
\zeta=(1-h) /\left(1+h \delta \xi_{0}\right) \text {. }
$$

Здесь $h=\frac{\partial \Psi}{d F_{0}}\left(F_{0}\right) ; \delta \xi_{0}=\Delta \xi_{0} / \xi$.

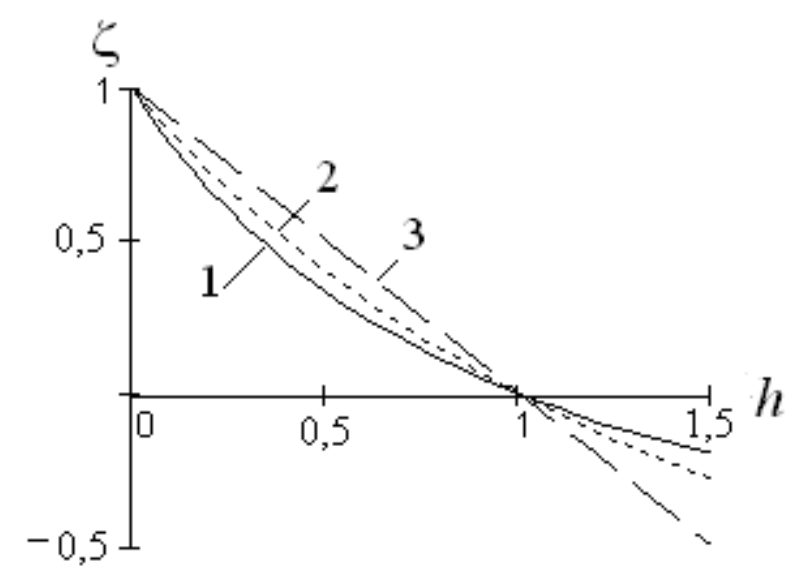

Рис. 4.10. График $\zeta(h): 1-\delta \xi_{0}=1 ; 2-\delta \xi_{0}=0,5 ; 3-\delta \xi_{0}=0$ 
График $\zeta(h)$ наглядно отображает три характерных режима работы системы замыкания (рис. 4.10). При малых значениях $h$ коэффициент $\zeta$ близок к единице. Это означает, что приращение коэффициента запаса $\Delta \xi$ лишь незначительно ниже своего идеального значения $\Delta \xi_{0}$. При значении $h \approx 1$ имеем $\zeta \approx 0$. Это соответствует случаю, когда увеличение замыкающего усилия не приводит к сколько-нибудь заметному изменению первоначального коэффициента запаса $\xi$. Наконец, при $h>1$ параметр $\zeta<0$. В этом режиме можно ожидать, что увеличение замыкающего усилия приведет к возрастанию интенсивности разрывов кинематической связи и дальнейшему ухудшению работы механизма. Это означает, что попытка устранить разрывы кинематического контакта за счет увеличения замыкающего усилия будет безуспешной, а система замыкания полностью лишается чувствительности к монтажным и эксплуатационным регулировкам, т. е. по сути дела оказывается неуправляемой.

Как следует из приведенных графиков, на реальном диапазоне изменения $\delta \xi_{0}=\Delta \xi_{0} / \xi<1$ этот параметр относительно мало влияет на значение $\zeta$.

Теперь обратимся к некоторым «профилактическим» мерам, исключающим возможность работы механизма в зоне нечувствительности замыкающей системы. Ограничим изменение параметра $\zeta$ некоторым допускаемым значением $\zeta<[\zeta]$.

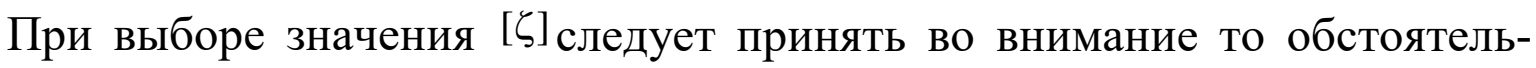
ство, что замыкающее усилие, препятствующее размыканию системы на выбеге, является весьма ощутимой дополнительной нагрузкой для звеньев и кинематических пар механизма на разбеге, что может привести к повышенному износу, а также понижает коэффициент полезного действия механизма, долговечность и надежность механизма. Поэтому представляется целесообразным принять $[\zeta] \approx 0,8$. Этому ограничению при учете (4.13) можно придать следующий вид

$$
h<[h]=(1-[\zeta]) /\left(1+\delta \xi_{0}\right) .
$$

Для устранения эффекта неуправляемости силовым замыканием, оставаясь в рамках принятой динамической модели, можно показать, что в первом приближении

$$
h \approx(3 \div 4)\left|\frac{P_{*}^{i} \sigma_{*}^{2} \Pi_{\max }^{\prime \prime}}{\left(\sigma_{*}^{2}-1\right) c_{1}}\right| .
$$

Здесь $c_{1}$ - приведенная крутильная жёсткость привода; $\sigma_{*}=p_{2} / p_{1}$, где $p_{1}, p_{2}$ - парциальные частоты ведущей и ведомой подсистем механизма; $P_{*}^{i}-$ идеальная сила инерции (или момент инерционных сил) в зоне возможного разрыва кинематической цепи.

Из зависимости (4.15) следует, что отмеченное явление не может быть устранено за счет изменения параметров замыкающей пружины. Оно определяется динамическими характеристиками привода и, в первую очередь, его 
приведенной жесткостью, которая должна удовлетворять следующему условию:

$$
c_{1}>c_{1}^{*} \approx \frac{20 \sigma_{*}^{2}\left(1+\delta \xi_{0}\right)}{\left|\sigma_{*}^{2}-1\right|}\left|\Pi^{\prime \prime}\right|_{\max } P_{*}^{i} \xi
$$

При $c_{1} \rightarrow \infty$ согласно (4.15) имеем $h \rightarrow 0$; при этом действительное приращение коэффициента запаса совпадает с идеальным, т.е. $\zeta \rightarrow 0$ (см рис.4.10).

Итак, увеличение замыкающего усилия в целях преодоления инерционных нагрузок выходного звена при определенных условиях может оказаться неэффективным, так как одновременно растет вызванная этим усилием сила инерции. Это приводит к неуправляемости системой замыкания и к повышению виброактивности системы. Ниже эта задача рассмотрена совместно с анализом колебаний в самой замыкающей пружине, что даёт возможность для более полной оценки исследуемого явления и соответствующих инженерных рекомендаций [10] .

\section{4. Продольные колебания замыкающих пружин}

Итак, увеличение замыкающего усилия в целях преодоления инерционных нагрузок выходного звена при определенных условиях может оказаться неэффективным, так как одновременно растет вызванная этим усилием сила инерции. Это приводит к неуправляемости системой замыкания и к повышению виброактивности системы. Далее данную задачу рассмотрим совместно с анализом колебаний в самой замыкающей пружине, что даёт возможность для более полной оценки исследуемого явления и соответствующих инженерных рекомендаций.

Обратимся к динамической модели (рис.4.11), которая отображает цикловой механизм, представленный в виде последовательной цепочки инерционных $\left(J_{0}, J_{1}, m\right)$ и упругодиссипативных $\left(c_{1}, \psi_{1}\right)$ элементов.

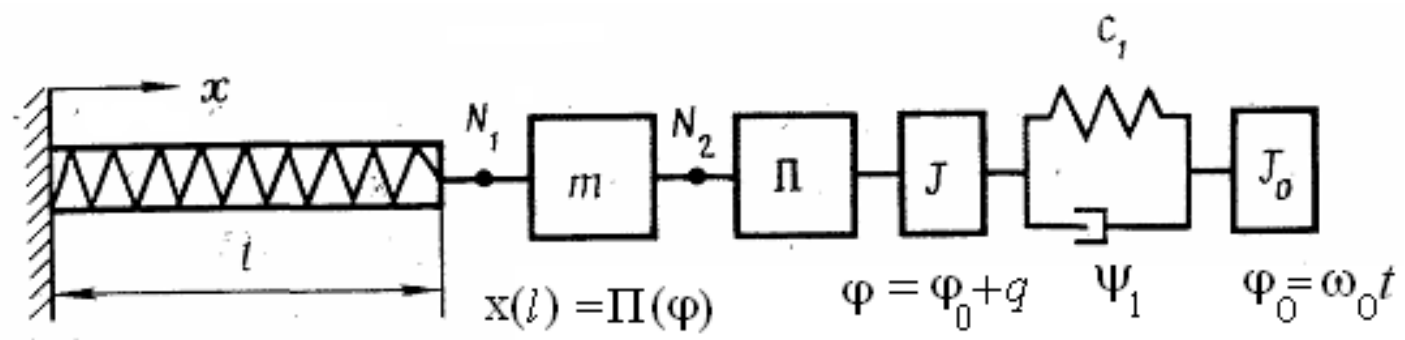

Рис.4.11. Модель циклового механизма с пружинным замыканием

Входная часть механизма $J$ отделена от выходной $m$ элементом $\Pi$, имитирующим преобразование входной координаты $\varphi_{0}$ в выходную $s=\Pi\left(\varphi_{0}\right)$. Угловая скорость элемента $J_{0}$ предполагается постоянной $\omega_{0}$. Замыкающая пружина, присоединенная к выходному звену в точке $N_{1}$, строго говоря, представляет собой пространственный кривой брус, однако при сравнительно ма- 
лых углах подъема винтовой линии пружину можно заменить прямым стержнем.

При определении частотных характеристик системы можно исключить из рассмотрения пренебрежимо малое влияние диссипации. Запишем однородное дифференциальное уравнение схематизированной пружины

$$
\frac{\partial^{2} u}{\partial x^{2}}-g_{0}^{-2} \frac{\partial^{2} u}{\partial t^{2}}=0
$$

где $u(x, t)$ - перемещение сечения $x ; g_{0}^{2}=E S / \mu_{0} ; E S ; \mu_{0}$-жесткость сечения эквивалентного «стержня» и его погонная масса; $E$ - модуль упругости; $S$ - площадь поперечного сечения стержня.

Принимая, что длина эквивалентного стержня равна средней высоте пружины $\ell$, для цилиндрической винтовой пружины из стальной проволоки круглого сечения в системе единиц СИ имеем $E S=8 \cdot 10^{10} d^{4} \ell / 8 D^{3} n$; $g_{0}=2,264 \cdot 10^{3} d \ell /\left(\pi D^{3} n\right)$, где $d-$ диаметр проволоки, $D-$ средний диаметр пружины, $n-$ число рабочих витков.

Поскольку параметры реальных механизмов являются медленно меняющимися функциями времени, одно из граничных условий уравнения (4.16) также носит нестационарный характер. Согласно методу условного осциллятора $[5,10,13]$ частное решение уравнения (4.16) может быть представлено в виде, $\quad u=X(x, \tau) \Psi(t)$, где $\tau$-медленное время .

Тогда уравнению (4.16) соответствует следующее обыкновенное дифференциальное уравнение, описывающее нестационарную форму колебаний

$$
X^{\prime \prime}+\lambda^{2}(\tau) X=0
$$

где ()$^{\prime}=\partial / \partial x ; \lambda^{2}(\tau)=\mu_{0} p^{2}(\tau) /(E S) ; p(\tau)$ - переменная «собственная» частота.

Приближенное решение уравнения (4.17) запишем в виде

$$
X=\alpha \cos \lambda x+\sin \lambda x
$$

Поскольку $X(0, \tau) \equiv 0$, имеем $\alpha=0$. Кроме того, нормируя форму таким образом, чтобы $X(\ell, \tau) \equiv 1$, представим (4.18) как

$$
X=\sin \theta / \sin \theta_{*},
$$

где $\theta=\lambda x, \theta_{*}=\lambda \ell$.

Неиспользованное второе граничное условие определяется на основании равенства амплитудных значений сил в сечении $N_{1}$ при учете $u=-s=-\Pi(\varphi)$ :

$$
\ell X^{\prime}\left(\theta_{*}\right) / X\left(\theta_{*}\right)=-R / c_{0},
$$

где $c_{0}=E S / \ell-$ коэффициент жесткости пружины; $R$ - динамическая жесткость механизма.

На основании (4.19), (4.20) после определения динамической жёсткости механизма формальное частотное уравнение может быть записано следующим образом: 


$$
\frac{\operatorname{tg} \theta_{*}}{\theta_{*}}\left[\frac{\theta_{*}^{2} g_{0}^{2}\left(J+m \Pi_{*}^{\prime 2}\right)}{c_{1} \ell^{2}}-1\right]=\frac{c_{0} \Pi_{*}^{\prime 2}}{c_{1}} .
$$

Уравнению (4.21) отвечает бесконечное число корней $\theta_{* r}(\tau)$ $r=(\overline{1, \infty})$. Переменная «собственная» частота находится как $p_{r}(\tau)=g_{0} \theta_{* r}(\tau) / \ell$. С учетом конкретных условий рассматриваемой задачи обычно нет необходимости решать трансцендентное уравнение (4.21). Дело в том, что в правой части уравнения стоит член $\varepsilon=c_{0} \Pi_{*}^{\prime 2} / c_{1}$ равный отношению приведенного к входному звену коэффициента жесткости замыкающей пружины к коэффициенту жесткости этого звена. Очевидно, что при реальных значениях параметров, как правило, $\varepsilon \square 1$. Если в качестве первого приближения принять $\varepsilon=0$, то уравнение (4.21) распадается на два уравнения, корни которых можно найти из простых зависимостей

$$
\theta_{* r}=\sqrt{c_{1} /\left(J+m \Pi_{*}^{\prime 2}\right)}, \quad \theta_{* r+1}=r \pi, \quad(r=\overline{1, \infty}) .
$$

Таким образом, практически в данной модели только одна из частот (обычно низшая) изменяется во времени, а остальные не зависят ни от времени, ни от параметров механизма. Этим обстоятельством мы воспользуемся при определении вынужденных колебаний системы.

Итак, проведенный частотный анализ дает основание для обособленного рассмотрения вынужденных колебаний пружины, т. е. декомпозиции исходной системы. Конец пружины перемещается по закону $u=-\Pi\left(\varphi_{0}+q\right) \approx-\Pi\left(\varphi_{0}\right)-\Pi^{\prime}\left(\varphi_{0}\right) q$. Однако при малых колебаниях $\Pi^{\prime} q \square \Pi_{\max }$, поэтому можно исходить из предположения, что сечение $x=\ell$ перемещается по идеальному закону движения, который представим в виде ряда Фурье

$$
u(\ell, t)=b_{0}+\sum_{j=1}^{\infty} b_{j} \sin \left(j \omega t+\gamma_{j}\right)
$$

При решении данной задачи нельзя непосредственно применять метод Фурье, так как граничные условия неоднородны. Эта трудность устраняется с помощью подстановки $u=u_{1}+w$, где $w=\kappa_{1}(t)+\left[\kappa_{2}(t)-\kappa_{1}(t)\right] x / \ell$; $\kappa_{1}=u(0, t) ; \quad \kappa_{2}(t)=u(\ell, t)$ (в нашем случае $\kappa_{2}=0$ ). Для новой переменной $u_{1}$ имеем нулевые граничные условия: $u_{1}(0, t)=0 ; u_{1}(\ell, t)=0$, однако уравнение становится неоднородным:

$$
\frac{\partial^{2} u_{1}}{\partial x^{2}}-g_{0}^{-2} \frac{\partial^{2} u_{1}}{\partial t^{2}}=\frac{x}{g_{0}^{2} \ell} \frac{d^{2} \kappa_{2}}{d t^{2}} .
$$

Решение ищем в виде 


$$
u_{1}=\sum_{j=1}^{\infty} v_{j}(x) \sin \left(j \omega t+\gamma_{j}\right)
$$

После подстановки (4.25) в (4.24), учета граничных условий и перехода к исходной переменной окончательно получаем

$$
u=\sum_{j=1}^{\infty} b_{j} \sin \left(j \omega t+\gamma_{j}\right) \sin \left(j \omega x / g_{0}\right) / \sin \left(j \omega \ell / g_{0}\right)+b_{0} x / \ell
$$

Замыкающая сила при этом определяется как $F=(E S)_{0} \frac{\partial u}{\partial x}(\ell)$. При этом

$$
F=\chi_{1} \omega \sum_{j=1}^{\infty} j b_{j} \operatorname{ctg}\left(j \omega \ell / g_{0}\right) \sin \left(j \omega t+\gamma_{j}\right)+\chi_{2} b_{0},
$$

где для пружин из стальной проволоки

$\chi_{1}=1,387 \cdot 10^{7} d^{3} / D \quad \kappa г / \mathrm{c} ; \chi_{2}=10^{10} d^{4} /\left(D^{3} n\right) \quad \kappa г / \mathrm{c}^{2} \quad(d, D \quad$ в $\quad$ м; $n-$ число витков).

Условия силового замыкания будут соблюдены, если на всем кинематическом цикле $F<0$ для пружин сжатия и $F>0$ - для пружин растяжения, что эквивалентно требованию

$$
\chi_{1} \omega \sum_{j=1}^{\infty} j b_{j}\left|\operatorname{ctg}\left(j \omega \ell / g_{0}\right)\right|<\chi_{2}\left|b_{0}\right| .
$$

При $j \omega \ell / g_{0}=\pi s(s=\overline{1, \infty})$ в предположении отсутствия диссипации имеет место бесконечное возрастание левой части этого неравенства, что соответствует резонансной частоте $j \omega=k=s \frac{\pi g_{0}}{\ell}=2,264 \cdot 10^{3} \frac{d}{D^{2} n}$. Однако нормальное функционирование замыкающего устройства нарушается еще на подступах к резонансной зоне. Так, при моногармоническом характере закона движения $(j=1)$, принимая $\left|b_{0}\right|=\xi b_{1}$, где $\xi>1-$ исходный коэффициент запаса, получаем условие

$$
\xi>\chi_{1} \omega\left|\operatorname{ctg}\left(\omega \ell / g_{0}\right)\right| / \chi_{2} .
$$

При $\omega \rightarrow 0$ после раскрытия неопределенности формула (4.29) дает исходный результат $\xi>1$. Далее с ростом $\omega$ правая часть неравенства уменьшается, достигая при $\omega \ell / g_{0}=\pi / 2$ нулевого значения. Это повышение коэффициента запаса связано с эффектом динамической разгрузки (см.п.3.6). При дальнейшем увеличении ш по мере приближения к резонансному режиму $\omega=\pi g_{0} / \ell$ правая часть неравенства растет, что в конечном итоге приводит к нарушению условия (4.29). Это свидетельствует о том, что пружина перестает осуществлять силовое замыкание.

Если принять, что в цикловом механизме доминирует динамическая нагрузка, связанная с неравномерным движением массы $m$, то для исклю- 
чения размыкания в сечении $N_{2}$ (см.рис.4.11) условие (4.29) должно быть ужесточено

$$
F_{0}>\chi_{1} \omega_{0} \sum_{j=4}^{\infty} j\left|\operatorname{ctg}\left(j \omega_{0} \ell / g_{0}\right)\right|-m w
$$

где $w$ - ускорение массы $m$, которое зависит как от ускорения программного движения, так и от колебаний $q$ входного звена $F_{0}=\chi_{0}\left|b_{0}\right|$.

Для определения $q(t)$ можно воспользоваться усеченной моделью, базирующейся на выявленной выше возможности декомпозиции системы. Эта модель, отображающая колебательную систему привода с переменным моментом инерции $J+m \Pi_{*}^{\prime 2}$. описывается следующим линейным дифференциальным уравнением с переменными коэффициентами :

$$
\left(J+m \Pi_{*}^{\prime 2}\right) \ddot{q}+\left(\beta+2 m \omega_{0} \Pi_{*}^{\prime} \Pi_{*}^{\prime \prime}\right) \dot{q}+c_{0} q=\Pi_{*}^{\prime}\left[F(t)-m \omega_{0}^{2} \Pi^{\prime \prime}\right],
$$

где $\beta$ - коэффициент эквивалентного линейного сопротивления; звездочка соответствует аргументу $\varphi_{0}=\omega_{0} t ; \quad F(t)$ задается формулой (4.27).

В дальнейшем предполагается, что параметры системы отвечают условиям динамической устойчивости как в зонах параметрических резонансов, так и на конечном отрезке кинематического цикла. Достаточное условие динамической устойчивости приводится к виду $\beta>p_{1}\left(J+m \Pi_{*}^{\prime 2}\right) \cdot d p_{1} / d t$.

Приближенное аналитическое решение уравнения, полученное с помощью метода условного осциллятора приведено в [10]. При известном решении $q(t)$ ускорение описывается зависимостью

$$
w=\Pi^{\prime \prime} \omega_{0}^{2}\left(1+\dot{q} / \omega_{0}\right)^{2}+\Pi^{\prime} \ddot{q},
$$

после чего неравенство (4.30) может быть проверено для любого текущего момента времени. Нарушение этого неравенства в первую очередь следует ожидать в окрестности значений углов поворота $\varphi_{0}$, отвечающих минимуму второй геометрической передаточной функции П". Согласно (4.27) и (4.30) при увеличении $F_{0}$ возрастает и вынуждающая сила, а следовательно, и экстремальные значения ускорений толкателя $w(t)$.

Наряду с приведенной выше проверкой желательно в процессе синтеза замыкающей системы располагать оценочным условием, которое могло бы служить ориентиром при проектировании и материалом для дальнейших уточнений. С этой целью, используя принцип суперпозиции, выделим из решения $q(t)$ составляющую, пропорциональную среднему значению замыкающей силы

$$
q=q_{0}+F_{0} \sum_{j=1}^{\infty} D_{j} \cos \left(j \omega_{0} t+\alpha\right) .
$$


Как показывает анализ, для наиболее «сильных» гармоник при некотором удалении от резонансных зон $D_{j} \approx \kappa_{j} j b_{j} / c_{1}$, где $\kappa_{j} \approx\left[1-\left(j \omega_{0} / \bar{p}_{1}\right)^{2}\right]^{-1} ; \bar{p}_{1}-$ среднее значение частоты $p_{1}$.

Далее, принимая во внимание, что в реальных механизмах $\dot{q} / \omega_{0}<0,1$, произведем линеаризацию функции (4.32)

$$
w \approx \Pi_{*}^{\prime \prime} \omega_{0}^{2}\left(1+2 \dot{q} / \omega_{0}\right)+\Pi_{*}^{\prime} \ddot{q} .
$$

Отсюда, согласно (4.32), (4.33), (4.34), с некоторым запасом имеем

$$
F_{0}=\frac{\chi_{1} \omega_{0} \sum_{j=1}^{\infty} j b_{j}\left|\operatorname{ctg}\left(j \omega_{0} \ell / g_{0}\right)\right|-K \Pi_{\min }^{\prime \prime} \omega_{0}^{2} m}{1-2\left|\Pi_{\text {min }}^{\prime \prime}\right| \omega_{0}^{2} m \sum_{j=1}^{\infty} j^{2} D_{j}(1+0,5 j N)},
$$

где $K=\mid w_{\min }^{0} /\left(\Pi_{\min }^{\prime \prime} \omega_{0}^{2}\left|; \quad w^{0}=w\left(q_{0}\right) ; \quad N=\right| \Pi_{* *}^{\prime} / \Pi_{\min }^{\prime \prime} \mid ; \quad \Pi_{* *}^{\prime}-\right.$ значение функции $\Pi^{\prime}$, отвечающее положению, при котором $\Pi^{\prime \prime}=\Pi_{\min }^{\prime \prime}$.

Если сохранить в функциях П' и П"одну доминирующую гармонику $v, \Delta \dot{q} \approx-F_{0} v b_{v} \kappa_{v} \omega_{0} c_{1}^{-1} \sin \left(v \omega_{0} t+\alpha_{v}\right), \quad \Delta \ddot{q} \square F_{0} v^{2} b_{v} \kappa_{v} \omega_{0}^{2} c_{1}^{-1} \cos \left(v \omega_{0} t+\alpha_{v}\right)$, где $\Delta q=q-q_{0}$.

При этом получаем

$$
F_{0}>\frac{\chi_{1} \omega_{0} \sum_{j=1}^{\infty} j b_{j}\left|\operatorname{ctg}\left(j \omega_{0} \ell / g_{0}\right)\right|-K \Pi_{\text {min }}^{\prime \prime} \omega_{0}^{2} m}{1-\kappa_{v} v^{2} b_{v}^{2} \omega_{0}^{2} m c_{1}^{-1}} .
$$

При существенном уменьшении знаменателя в формулах (4.35) и (4.36) значительно возрастает среднее значение $F_{0}$, необходимое для надежного силового замыкания, а при обращении знаменателя в нуль наступает эффект неуправляемости системой замыкания, физический смысл которого был разъяснен выше. Для исключения возможности возникновения указанного эффекта необходимо за счет выбора коэффициента крутильной жесткости $c_{1}$ удовлетворить условию, при котором знаменатели в этих формулах мало отличались бы от единицы. Например, для частного случая, отвечающего формуле (4.36), следует потребовать $c_{1} \square \kappa_{v} v^{2} b_{v}^{2} \omega_{0}^{2} m$. Величину, обратную значению знаменателя, можно рассматривать как критерий, характеризующий увеличение силы инерции за счет роста среднего значения замыкающего усилия.

\section{5. Поперечные колебания замыкающих пружин}

Система дифференциальных уравнений. При расчете поперечных колебаний пружины ее также заменяют эквивалентным стержнем. Однако в этом случае приходится принимать во внимание не только изгиб в плоскости витка, но также и крутильные, и продольные деформации элемента 
витка. С учетом сдвигов, инерции вращения сечений и продольных нагрузок система дифференциальных уравнений, описывающих поперечные колебания пружин, имеет вид

$$
\begin{aligned}
& \frac{\partial^{2} y}{\partial x^{2}}-\frac{m}{S_{2}} \frac{\partial^{2} y}{\partial t^{2}}-\left(1-\frac{F}{S_{2}}\right) \frac{\partial \theta}{\partial x}=0 \\
& \frac{\partial^{2} y}{\partial x^{2}}-\frac{\rho}{S_{1}} \frac{\partial^{2} \theta}{\partial x^{2}}+\frac{S_{2}}{S_{1}}\left(1-\frac{F}{S_{2}}\right)\left(\frac{\partial y}{\partial x}-\theta\right)=0,
\end{aligned}
$$

где $y$ - поперечное перемещение; $\theta$ - средний угол поворота поперечного сечения; $m, \rho-$ масса и массовый момент инерции единицы длины эквивалентного стержня; $F$-продольная нагрузка $(F>0$ - растяжение; $F<0$ - сжатие); $S_{1}, \quad S_{2}-$ изгибная и сдвиговая жесткости сечения эквивалентного стержня.

Для пружин из круглой проволоки

$$
m=\rho_{0} \frac{\pi^{2} D d^{2} n}{4 l} ; \rho=\rho_{0} \frac{\pi^{2} D^{3} d^{2} n}{32 l} ; S_{1}=\frac{2 \ell E I}{\pi D n\left(2+\mu_{\mathrm{II}}\right)} ; S_{2}=\frac{8 \ell E I}{\pi D^{3} n},
$$

где $D$ - диаметр проволоки; $\ell$ - высота пружины при средней нагрузке; $d$ - диаметр проволоки; $\rho_{0}$ - плотность материала; $E$ - модуль сдвига; $I=\pi d^{4} / 64-$ момент инерции сечения; $\mu_{п}-$ коэффициент Пуассона (для стали $\left.\mu_{\Pi}=0,3\right) ; n-$ число рабочих витков.

При использовании пружины для силового замыкания кинематических пар ее концы нередко присоединены к подвижным звеньям. В этом случае граничные условия оказываются заданными функциями времени. Пусть $y(0, t)=\kappa_{\mathrm{I}}(t), \quad y(\ell, t)=\kappa_{2}(t) \quad\left(\kappa_{\mathrm{I}} \square \ell, \kappa_{2} \square \ell\right)$. Можно было бы также потребовать $\theta(0, t)=\kappa_{3}(t), \theta(\ell, t)=\kappa_{4}(t)$. Однако при проектировании механизмов обычно принимаются специальные меры для того, чтобы опорные поверхности не «выламывали» пружину, поэтому наличие подобных граничных условий свидетельствует о неудачном конструктивном решении. Другими словами, при подвижных концах замыкающей пружины следует предусмотреть возможность самоустановки плоскости торцов, что соответствует отсутствию изгибающих моментов в этих сечениях, т. е. шарнирному закреплению

Введём подстановку

$$
y=(x, t)=y_{1}(x, t)+w(x, t),
$$

где $w(x, t)=\kappa_{1}(t)+\left[\kappa_{2}(t)-\kappa_{1}(t)\right] x / \ell$.

Тогда $w(0, t)=\kappa_{1}(t), w(\ell, t)=\kappa_{2}(t), y_{1}(0, t)=y_{1}(\ell, t)=0$ и система дифференциальных уравнений приводится к виду

$$
\frac{\partial^{2} y_{1}}{\partial x^{2}}-\frac{m}{S_{2}} \frac{\partial^{2} y_{1}}{\partial t^{2}}-(1-f) \frac{\partial \theta}{\partial x}=\frac{m}{S_{2}}\left[\frac{d^{2} \kappa_{1}}{d t^{2}}+\left(\frac{d^{2} \kappa_{2}}{d t^{2}}-\frac{d^{2} \kappa_{1}}{d t^{2}}\right) \frac{x}{\ell}\right]
$$




$$
\frac{\partial^{2} \theta}{\partial x^{2}}-\frac{\rho}{S_{1}} \frac{\partial^{2} \theta}{\partial t^{2}}=-\frac{S_{2}}{S_{1}}(1-f)\left[\frac{\partial y_{1}}{\partial x}-\theta+\frac{\kappa_{2}-\kappa_{1}}{\ell}\right],
$$

где $f=F / S_{2}$.

Определение собственных частот и форм колебаний. Подставим в систему однородных дифференциальных уравнений, полученную из (4.39) при $\kappa_{1}=\kappa_{2}=0, \quad y_{1}=Y_{1}(x) \sin (k t), \quad \theta=v(x) \sin (k t)$. После сокращения на $\sin k t$ получаем следующую систему дифференциальных уравнений:

$$
\left.\begin{array}{l}
Y_{1}^{\prime \prime}+\frac{m k^{2}}{S_{2}} Y_{1}-(1-f) v^{\prime}=0 \\
\frac{S_{2}}{S_{1}}(1-f) Y_{1}^{\prime}+v^{\prime \prime}+\left[\frac{\rho k^{2}}{S_{1}}-\frac{S_{2}}{S_{1}}(1-f)\right] v=0,
\end{array}\right\}
$$

где ()$^{\prime}=\partial / \partial x$

Полагая $Y_{1}=A \mathrm{e}^{\lambda x} \quad$ и $\quad v=B \mathrm{e}^{\lambda x}$, трансформируем (4.39) в однородную систему алгебраических уравнений относительно А и В. Далее обращая в нуль определитель этой системы, получаем характеристическое уравнение

$$
\lambda^{4}+2 b_{1}^{2} \lambda^{2}-b_{2}^{4}=0
$$

где $\quad b_{1}^{2}=0,5\left[k^{2}\left(\frac{m}{S_{2}}+\frac{\rho}{S_{1}}\right)-\frac{S_{2}}{S_{1}} f(1-f)\right] ; b_{2}^{4}=\frac{m k^{2}}{S_{1}}\left(1-f-\frac{\rho k^{2}}{S_{2}}\right)$.

Отсюда следует $\quad \lambda_{1,2}= \pm i \alpha_{1}(i=\sqrt{-1}) ; \quad \lambda_{3,4}= \pm \alpha_{2}, \quad$ причём

$$
\left.\begin{array}{c}
\alpha_{1}=\sqrt{b_{1}^{2}+\sqrt{b_{1}^{4}+b_{2}^{4}}} ; \alpha_{2}=\sqrt{-b_{1}^{2}+\sqrt{b_{1}^{4}+b_{2}^{4}}} \text {. Тогда } \\
Y_{1}=C_{1} \cos \left(\alpha_{1} x\right)+C_{2} \sin \left(\alpha_{1} x\right)+C_{3} \operatorname{ch}\left(\alpha_{2} x\right)+C_{4} \operatorname{sh}\left(\alpha_{2} x\right) ; \\
v^{\prime}=\beta_{1}\left[C_{1} \cos \left(\alpha_{1} x\right)+C_{2} \sin \left(\alpha_{1} x\right)\right]+\beta_{2}\left[C_{3} \operatorname{ch}\left(\alpha_{2} x\right)+C_{4}\left(\operatorname{sh} \alpha_{2} x\right)\right],
\end{array}\right\}
$$

где $\beta_{1}=\left(m k^{2} S_{2}^{-1}-\alpha_{1}^{2}\right) /(1-f) ; \quad \beta_{2}=\left(m k^{2} S_{2}^{-1}+\alpha_{2}^{2}\right) /(1-f)$.

Константы $C_{1}, C_{2}, C_{3}, C_{4}$ находим из граничных условий, которые при шарнирном закреплении концов пружины имеют вид $Y_{1}(0)=Y_{1}(\ell)=0$ (отсутствие прогиба); $v^{\prime}(0)=v^{\prime}(\ell)=0$ (отсутствие изгибающего момента). Опуская выкладки, на основании (4.42) получаем следующее частотное уравнение:

$$
\zeta_{1} \tilde{k}^{4}-z^{2}\left[z^{2}(1-f)+\zeta_{2}\right] \tilde{k}^{2}+z^{4}\left[1+\zeta_{3} z^{2} f(1-f)\right]=0
$$

где $\tilde{k}=k / k_{0} ; \quad k_{0}=j^{2} \pi^{2} \ell^{-2} \sqrt{S_{1} / m ;} \quad z=\ell / D$ (индекс пружины).

Для пружин из стальной круглой проволоки $\zeta_{1}=1,32 ; \zeta_{2}=2,31 ; \zeta_{3}=0,932$.

Как следует из анализа, растягивающее усилие $(F>0, f>0)$ повышает низшую собственную частоту, а сжимающее $(F<0, f<0)$ - понижает. 
При $\tilde{k} \leq 0$ прямолинейная форма равновесия пружины становится неустойчивой. Очевидно, что соответствующее критическое значение силы сжатия пружины $F_{*}=f_{*} S_{2}$ отвечает обращению в нуль свободного члена уравнения (4.43); при этом $f_{*}$ определяется как отрицательный корень квадратного уравнения

$$
\zeta_{3} z^{2} f_{*}^{2}-\zeta_{3} z^{2} f_{*}-1=0 .
$$

Для определения форм колебаний при принятых граничных условиях следует в (4.42) подставить соответствующее значение $k$ при $C_{1}=C_{3}=0$; $C_{2}=1 ; \quad C_{4}=-\sin \alpha_{1} \ell / \operatorname{sh} \alpha_{2} \ell$.

При заделанных торцевых сечениях пружины имеем следующие граничные условия: $Y_{1}(0)=Y_{1}(\ell)=0 \quad$ (отсутствие прогиба); $\mathrm{v}(0)=\mathrm{v}(\ell)=0 \quad$ (отсутствие угловой деформации в торцевом сечении пружины). При этом частотное уравнение оказывается трансцендентным:

$$
\left|\begin{array}{cc}
\cos \alpha_{1} \ell+\operatorname{ch} \alpha_{2} \ell & \sin \alpha_{1} \ell+\frac{\alpha_{2} \beta_{1}}{\alpha_{1} \beta_{2}} \operatorname{sh} \alpha_{2} \ell \\
\frac{\beta_{1}}{\alpha_{1}} \sin \alpha_{1} \ell-\frac{\beta_{2}}{\alpha_{2}} \operatorname{sh} \alpha_{2} \ell & \frac{\beta_{1}}{\alpha_{1}}\left(\operatorname{ch} \alpha_{2} \ell-\cos \alpha_{1} \ell\right.
\end{array}\right|=0 .
$$

Критическое значение параметра $f_{*}<0$, отвечающее потере устойчивости прямолинейной формы равновесия пружины, определяется из (4.43)(4.45) при подстановке $k=0$.

Вынужденные колебания при кинематическом возбуждении. Пусть концы пружины совершают перпендикулярно к ее оси относительно малые перемещения $\kappa_{1}=a_{1} \sin \omega t, \kappa_{2}=a_{2} \sin \omega t \quad\left(a_{1} \square \ell, a_{2} \square \ell\right)$. Представим решение в форме $y_{1}(x, t)=Y_{\omega}(x) \sin \omega t, \theta(x, t)=\mathrm{v}_{\omega}(x) \sin \omega t$. После подстановки этих функций в (4.39) имеем

$$
\begin{aligned}
& Y_{\omega}^{\prime \prime}+\frac{m \omega^{2}}{S_{2}} Y_{\omega}-(1-f) \mathrm{v}_{\omega}=\frac{m \omega^{2}}{S_{2}}\left[a_{1}+\left(a_{2}-a_{1}\right) \frac{x}{\ell}\right] ; \\
& \frac{S_{2}}{S_{1}}(1-f) Y_{\omega}^{\prime}+\mathrm{v}_{\omega}^{\prime \prime}+\left[\frac{\rho \omega^{2}}{S_{1}}-\frac{S_{2}}{S_{1}}(1-f)\right] \mathrm{v}_{\omega}=\frac{\left(a_{1}-a_{2}\right)(1-f) S_{2}}{S_{1} \ell}, \\
& \text { где }()^{\prime}=\partial / \partial x . \\
& \text { Решение системы }(4.46) \text { имеет вид } \\
& Y_{\omega}=h_{1} \cos \alpha_{1} x+h_{2} \sin \alpha_{1} x+h_{3} \operatorname{ch} \alpha_{1} x+h_{4} \operatorname{sh} \alpha_{2} x+h_{5} x+h_{6} ; \\
& \mathrm{v}_{\omega}=\beta_{1} \alpha_{1}^{-1}\left(h_{1} \sin \alpha_{1} x-h_{2} \cos \alpha_{1} x\right)+\beta_{2} \alpha_{2}^{-1}\left(h_{3} \operatorname{sh} \alpha_{1} x+h_{4} \operatorname{ch} \alpha_{2} x\right),
\end{aligned}
$$

где $h_{i}(i=\overline{1,6})$ - некоторые константы.

Функции $\beta_{1}, \beta_{2}, \alpha_{1}, \alpha_{2}$ определяются по приведенным выше формулам при замене собственной частоты $k$ на частоту кинематического возбуждения $\omega$.

Последние два слагаемых в зависимости для $Y_{\omega}$ соответствуют частному решению, подстановка которого в (4.39) дает $h_{5}=\left(a_{2}-a_{1}\right) / \ell, \quad h_{6}=a_{1}$. 
Остальные константы находим из граничных условий для опертых концов пружины $Y_{\omega}(0)=Y_{\omega}(\ell)=0 ; \mathrm{v}_{\omega}^{\prime}(0)=\mathrm{v}_{\omega}^{\prime}(\ell)=0$. Из первого уравнения системы (4.46) непосредственно следует, что последние два граничных условия эквивалентны $Y_{\omega}^{\prime \prime}(0)=m \omega^{2} a_{1} / S_{2}$ и $Y_{\omega}^{\prime \prime}(\ell)=m \omega^{2} a_{2} / S_{2}$. . На основании (4.47) получаем

$$
\begin{array}{ll}
h_{1}=-\frac{a_{1} \alpha_{2}^{2}}{\alpha_{1}^{2}+\alpha_{2}^{2}} ; & h_{2}=\frac{\alpha_{2}^{2}\left(a_{1} \cos \alpha_{1} \ell-a_{2}\right)}{\left(\alpha_{1}^{2}+\alpha_{2}^{2}\right) \sin \alpha_{1} \ell} ; \\
h_{3}=-\frac{a_{1} \alpha_{1}^{2}}{\alpha_{1}^{2}+\alpha_{2}^{2}} ; & h_{4}=\frac{\alpha_{1}^{2}\left(a_{1} \operatorname{ch} \alpha_{2} \ell-a_{2}\right)}{\left(\alpha_{1}^{2}+\alpha_{2}^{2}\right) \operatorname{sh} \alpha_{2} \ell} .
\end{array}
$$

При резонансе $\omega=k$ имеем $\sin \alpha_{1} \ell=0$; тогда при условии, что, $a_{1} \neq a_{2}, h_{2} \rightarrow \infty$ и $Y_{\omega} \rightarrow \infty$. Если, $a_{1}=a_{2}$, то при резонансе возбуждаются только симметричные формы колебаний, т.е. $a_{1} \ell=(2 j-1) \pi$. Если же кинематическое возбуждение концов пружины имеет одинаковые амплитуды, но сдвиг по фазе равен $\pi\left(a_{1}=-a_{2}\right)$,то резонансу отвечает $\alpha_{1} \ell=2 j \pi$; при этом возбуждаются только антисимметричные формы. Полное перемещение произвольного сечения пружины находим как

$$
y_{\omega}=\left[Y_{\omega}(x)+a_{1}+\left(a_{2}-a_{1}\right) x / \ell\right] \sin \omega t .
$$

При использовании рядов Фурье приведенные зависимости легко обобщаются на случай, когда функции $\kappa_{1}(t) u \kappa_{2}(t)$ - произвольные периодические функции.

Параметрические и комбинационные резонансы пружин. Как следует из (4.43), продольное возмущение, с которым связана переменность параметров пружины, приводит к пульсации «собственных» частот, что может служить причиной параметрического возбуждения. Основные параметрические резонансы возникают в окрестности усредненных частот $\omega_{*}=2 \bar{k}_{1,2}$, а комбинационные $-\omega_{*}=\bar{k}_{2} \pm \bar{k}_{1}$.

Специфика параметрического возбуждения пружин связана с малыми значениями логарифмического декремента $\lambda$ (обычно $\lambda<0,1$ ), поэтому обеспечение условий динамической устойчивости приходится осуществлять главным образом за счет отстройки от критических зон. Кроме того, следует иметь в виду, что продольная вынуждающая сила всегда приложена к пружине эксцентрично и не строго параллельна оси пружины, что приводит к неизбежному возбуждению поперечных колебаний. Последние могут вступать во взаимодействие с параметрическими колебаниями [16]. 


\section{ГЛАВА 5. СИНТЕЗ ЗАКОНОВ ДВИЖЕНИЯ РАБОЧЕГО ОРГАНА ПРИ УЧЕТЕ УПРУГОСТИ ПРИВОДА И ХАРАКТЕРИСТИК ЭЛЕКТРОДВИГАТЕЛЯ}

\section{1. Линеаризация геометрических характеристик циклового механизма в окрестности программного движения}

Рассмотрим наиболее простую динамическую модель механизма с нелинейной функцией положения, в которой привод представлен в виде колебательного контура с одной степенью свободы, а выходные звенья приняты абсолютно жёсткими (рис. 5.1). В этой модели кинематический аналог, роль которого выполняет нелинейная функция $x=\Pi(\varphi)$, «встроен» между инерционными элементами $J$ и $m$. Эта модель позволяет в первом приближении выявить динамические ошибки, возникающие из-за крутильных колебаний вала, передаточных механизмов и других элементов привода.

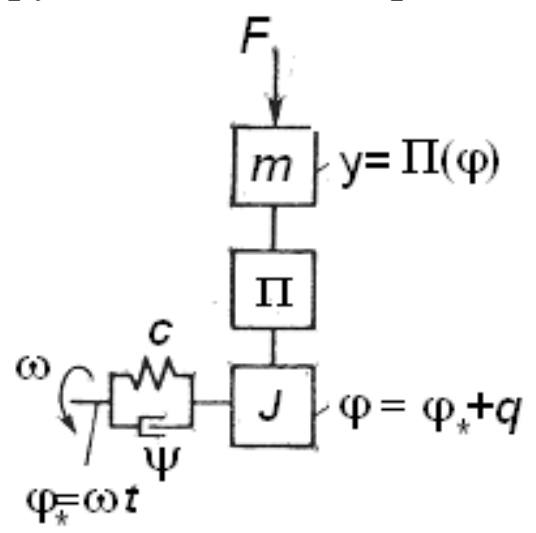

Рис. 5.1. Динамическая модель механизма при учете упругости привода

Примем следующие условные обозначения: $q$ - угловая деформация привода, приведённая к входному звену; $\varphi_{*}=\omega_{0} t-$ «идеальный» угол поворота входного звена; $\varphi=\varphi_{*}+q-$ угол поворота входного звена с учётом крутильных колебаний; $F-$ внешняя сила, приложенная к выходному звену, которую будем считать зависящей от времени $t$ и угла $\varphi ; c_{0}, \psi-$ коэффициенты жёсткости и рассеяния приводной части механизма;

Используя изложенную в главе 2 методику математического описания модели, запишем дифференциальное уравнение

$$
J \ddot{q}+b_{0} \dot{q}+c_{0} q=-\Pi^{\prime}(\varphi)(m \ddot{y}+F),
$$

где $\ddot{y}=\Pi^{\prime \prime}(\varphi)(\omega+\dot{q})^{2}+\Pi^{\prime} \ddot{q}$;

В уравнении (5.1) также учтён приведённый, диссипативный момент, принятый пропорциональным скорости $\dot{q}$ (связь между коэффициентом пропорциональности $b_{0}$ и коэффициентом рассеяния $\psi$ см. ниже). Дифференциальное уравнение (5.1) является нелинейным, так как обобщённая координата и её производная входят в качестве аргументов нелинейных функций. 
Покажем, что это уравнение ценой малосущественных в динамическом отношении упрощений может быть приведено к виду линейного дифференциального уравнения с переменными коэффициентами. Предварительно разобьём фазовый угол поворота $2 \pi$, соответствующий полному обороту входного

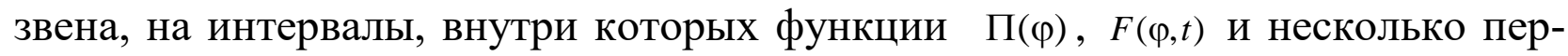
вых производных этих функций по $\varphi$ не терпят разрывов непрерывности. Тогда, раскладывая эти нелинейные функции в ряд Тейлора по степеням малых $q$, имеем

$$
\begin{aligned}
& \Pi^{\prime}(\varphi) \approx \Pi^{\prime}\left(\varphi_{*}\right)+\Pi^{\prime \prime}\left(\varphi_{*}\right) q ; \\
& \Pi^{\prime \prime}(\varphi) \approx \Pi^{\prime \prime}\left(\varphi_{*}\right)+\Pi^{\prime \prime \prime}\left(\varphi_{*}\right) q ; \\
& F(\varphi, t) \approx F\left(\varphi_{*} t\right)+\frac{\partial F}{\partial \varphi}\left(\varphi_{*} t\right) q
\end{aligned}
$$

Эту процедуру назовём линеаризацией в окрестности программного движсния. Не следует смешивать этот приём с такой линеаризацией, когда на выделенном интервале нелинейная функция заменяется линейной. В данном случае при относительно большом аргументе $\varphi_{*}=\omega t$ все функции сохранили свои нелинейные свойства, и лишь малые угловые деформации $q$ вошли в соответствующие выражения линейно. В частности, применительно к первой зависимости (5.2) это можно трактовать так: в малой окрестности текущего значения $\varphi_{*}$ кривую $\Pi^{\prime}(\varphi)$ можно заменить касательной с переменным угловым коэффициентом $\Pi^{\prime \prime}\left(\varphi_{*}\right)$. Указанное упрощение позволяет в дальнейшем пользоваться принципом суперпозиции (см.п.2.2.) справедливым лишь для линейных систем. Данные инженерных расчётов свидетельствуют о том, что, за исключением некоторых особых случаев, значения отброшенных членов рядов таковы, что не оправдывают дальнейших уточнений.

\section{2. Математическая модель. Условия устойчивости}

Математическая модель. После подстановки (5.2) в (5.1), сохраняя в полученных выражениях линейные члены относительно координаты $q$ и её производных, получаем

$$
a(t) \ddot{q}+b(t) \dot{q}+c(t) q=Q(t),
$$

где

$$
\begin{aligned}
& a(t)=J+m \Pi_{*}^{\prime 2} ; b(t)=b_{0}+2 m \omega \Pi_{*}^{\prime} \Pi_{*}^{\prime \prime} ; \\
& c(t)=c_{0}\left\{1+c_{0}^{-1} m \omega^{2}\left(\Pi_{*}^{\prime 2}+\Pi_{*}^{\prime} \Pi_{*}^{\prime \prime \prime}\right)+c_{0}^{-1}\left[\Pi_{*}^{\prime \prime} F_{x}+\Pi_{*}^{\prime}\left(\frac{\partial F}{\partial \varphi}\right)_{*}\right]\right\} ; \\
& Q(t)=-\Pi_{*}^{\prime}\left(m \omega^{2} \Pi_{*}^{\prime \prime}+F\right) .
\end{aligned}
$$

Здесь звёздочкой отмечены функции при аргументе $\varphi_{*}$. 
Нередко в выражении $c(t)$ значение функции, стоящей в фигурных скобках, мало отличается от единицы ( $c \approx c_{0}=$ const $)$, а доминирующую роль в формировании переменных «собственных» частот играют приведенные инерционные характеристики (функция $a(t)$ ). Для упругого валопровода с переменным приведённым моментом инерции $J(\varphi)$ (см. табл.6.1 модель 2) дифференциальное уравнение также приводится к виду (5.1) при

$$
a=J_{*} ; b=b_{0}+J_{*}^{\prime} ; c=c_{0}\left[1+\omega^{2} J^{\prime \prime} /\left(2 c_{0}\right)+M_{*}^{\prime} / c_{0}\right] ; Q(t)=-0,5 \omega^{2} J_{*}^{\prime}+M_{*} .
$$

Здесь $M_{*}=M\left(\varphi_{*}, t\right)-$ внешний момент; $J_{*}=J\left(\varphi_{*}\right) ; \quad c_{0}-$ коэффициент жёсткости валопровода; штрихом отмечена производная по $\varphi$.

Обратим внимание на двоякое происхождение коэффициента $b$, в котором первое слагаемое соответствует диссипативным факторам, а второе равно $d a / d t$, т.е связано с изменением инерционного коэффициента. Другим важным фактором, предопределяющим переменность параметров динамической модели, является изменение во времени приведённой жёсткости. Нередко это связано с переменностью углов давления [6, 22].

Приведем уравнение (5.3) к виду

$$
\ddot{q}+2 n(t) \dot{q}+k^{2}(t) q=w(t),
$$

где $n=b /(2 a) ; k^{2}=c / a ; w=Q / a-$ возмущение;

В таблице 5.1. приведены коэффициенты линеаризованных дифференциальных уравнений и абсолютные динамические ошибки, т.е. отклонения соответствующих координат от идеальных значений при отсутствии колебаний. В ряде коэффициентах выделена функция $\chi \square 1$, позволяющая упростить расчётные зависимости.

Таблица.5.1. Динамические ошибки и параметры математической модели

\begin{tabular}{|c|c|}
\hline Динамическая ошибки & $\begin{array}{c}\text { Коэффициенты } \\
\text { пифференциалного уравнения }\end{array}$ \\
\hline$\Delta y=\Pi\left(\varphi_{*}+q\right)$ & $k^{2}=\frac{c(1+\chi)}{J+m \Pi_{*}^{\prime 2}}$ \\
$\Delta \dot{y}=\omega_{0}\left[\left(1+\frac{\dot{q}}{\omega_{0}}\right) \times\right.$ & $n=\frac{\psi}{4 \pi} k+\frac{m \Pi_{*}^{\prime} \Pi_{*}^{\prime \prime} \omega_{0}}{J+m \Pi_{*}^{2}}$ \\
$\left.\times \Pi^{\prime}(\varphi)-\Pi^{\prime}\left(\varphi_{*}\right)\right]$ & $W=-\frac{\Pi_{*}^{\prime}\left(m \omega_{0}^{2} \Pi_{*}^{\prime \prime}+F_{*}\right)}{J+m \Pi_{*}^{\prime 2}}$ \\
$\Delta \ddot{y}=\omega_{0}^{2}\left[\left(1+\frac{\dot{q}}{\omega_{0}}\right)^{2} \times\right.$ & $\begin{array}{c}1 \\
\times \Pi^{\prime \prime}(\varphi)+\Pi^{\prime}(\varphi) \times \\
\left.\times \frac{\dot{q}}{\omega_{0}^{2}}-\Pi^{\prime \prime}\left(\varphi_{*}\right)\right]\end{array}$ \\
\hline
\end{tabular}


Проявление вибраций в механизмах, отображаемых динамическим моделями с переменными параметрами, является достаточно многообразным. Практически здесь наблюдаются те же динамические эффекты, с которыми мы сталкивались в моделях с постоянными параметрами. Поэтому при синтезе законов программного движения в первую очередь следует удовлетворить требования, изложенные в главах 2 и 3, принимая в расчетах усредненные значения параметров. Здесь остановимся лишь на некоторых эффектах, отражающих специфику систем с переменными параметрами.

Один из таких эффектов связан с возбуждением параметрических резонансов, когда возможен неограниченный рост амплитуд, при котором проблема повышения динамической точности, конечно, теряет смысл. Однако при этом также возможны локальные нарушения условий динамической устойчивости даже при медленном изменении параметров, т.е. на значительном удалении от основных резонансных зон.

Как показано в [5-11, 13] на основании метода условного осциллятора, при медленном изменении параметров $p(t) \approx k(t)$. При этом свободные колебания описываются зависимостью, которая совпадает с результатом, полученным с помощью ВКБ-приближения первого порядка [33].

$$
q=D_{0} \exp \left[-\int_{0}^{t} n(t)\right] \sqrt{k(0) / k(t)} \cos \left[\int_{0}^{t} k(t) d t+\alpha\right] .
$$

В системах с постоянными параметрами ( $n=$ const, $k=$ const $)$ формула (5.5) совпадает с формулой (2.24) и описывает затухающие свободные колебания, но при переменных параметрах даже при их медленном изменении амплитуда колебаний на отдельных временных отрезках может возрастать. Этот динамический эффект наглядно виден на экспериментальной записи (рис.5.2). Зона нарастания колебаний чередуется с зоной затухания, образуя своеобразную амплитудную модуляцию колебаний, которая существенно проявляется на уровне виброактивности машин и динамических ошибок законов движения исполнительных органов.

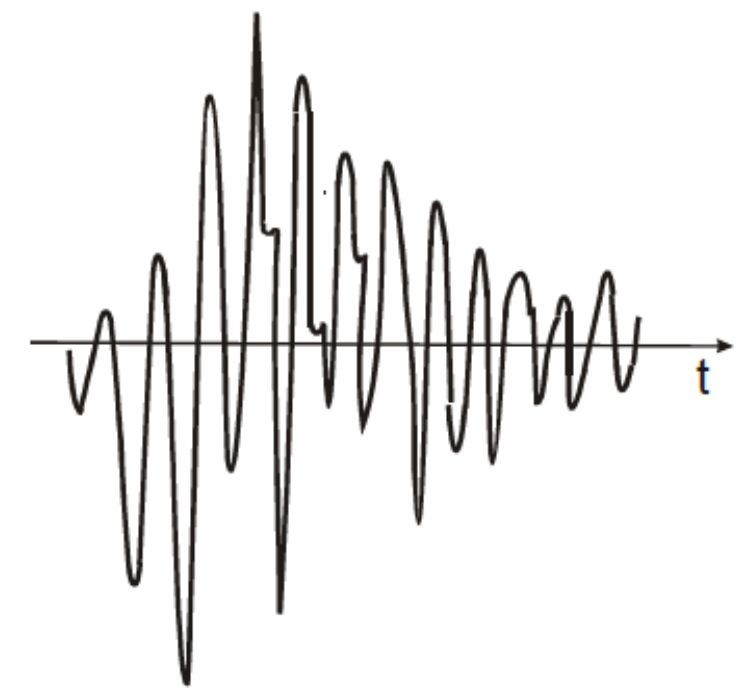

Рис.5.2. Амплитудная модуляция колебаний 
Условия устойчивости. Согласно (5.5) амплитуда колебаний при медленном изменении параметров пропорциональна функции

$$
U=\exp \left[-\int_{0}^{t} n(t) d t\right] / \sqrt{k(t),}
$$

где $n=\lambda k /(2 \pi)+0,5 \dot{a} / a ; \lambda-$ логарифмический декремент.

Чтобы исключить возможность увеличения амплитуд, потребуем

$$
d U / d t<0 .
$$

Неравенство (5.7) является достаточным условием динамической устойчивости на любом отрезке кинематического цикла_[8, 10, 11, 13].

После подстановки (5.6) в (5.7) получим

$$
n+0,5 \dot{k} / k>0 \text {. }
$$

Принимая во внимание, что $k^{2} \approx c / a$, приведем условие (5.8) к виду

$$
\lambda>-\frac{\pi \omega}{2 k(t)}\left(a^{\prime} / a+c^{\prime} / c\right) .
$$

Здесь $\omega$ - угловая скорость входного звена; штрихом, как и выше, обозначена производная по $\varphi$.

Аналогичные условия могут быть записаны для виброскоростей и виброускорений [10], [13].

При прочих равных условиях, чем выше отношение угловой скорости $\omega$ к «собственной» частоте $k(t)$, тем больше возможность появления зон нарастания амплитуд колебаний. Подчеркнем, что рассматриваемый эффект в отличие от параметрического резонанса не связан с определенной частотой параметрического возмущения.

Оценка резонансных амплитуд при совместном учете вынуждающей силы и параметрического возбуждения. Примем в дифференциальном уравнении (5.4) $k^{2}=k_{0}^{2}\left(1-\varepsilon \sin \omega_{2} t\right)$ и $W=h \sin \left(\omega_{1} t-\gamma_{1}\right)$, при $\omega_{2}=2 k_{0}=2 \omega_{1}$, что соответствует «силовому» резонансу и одновременно главному параметрическому резонансу. Запишем выражение, определяющее приращение энергии за период $T_{1}=2 \pi / \omega_{1}$ :

$$
\Delta E=\int_{E_{1}}^{E_{2}} d E=\int_{0}^{T_{1}}\left[h \dot{q} \sin \left(\omega_{1} t-\gamma_{1}\right)-2 n \dot{q}^{2}+\varepsilon k_{0}^{2} q \dot{q} \sin \omega_{2} t\right] d t .
$$

В установившемся режиме $\Delta E=0$. Отсюда, принимая в качестве приближенного решения $q=A \sin \left(\omega_{1} t-\gamma_{2}\right)$, после интегрирования получаем 


$$
A=\pi k_{0}^{-2} h \sin \left(\gamma_{2}-\gamma_{1}\right) /\left(\lambda-\lambda_{*} \cos 2 \gamma_{2}\right),
$$

где $\lambda_{*}=0,5 \pi \varepsilon$.

При резонансе $\gamma_{2}-\gamma_{1}=\pi / 2$. Таким образом, резонансная амплитуда А на основании (5.10) отвечает некоторой условной системе без параметрического возбуждения, но с измененным уровнем диссипации, соответствующим $\lambda_{1}=\lambda-\lambda_{*} \cos 2 \gamma_{2}$. В зависимости от фазового сдвига $\gamma_{2}$ этот параметр принимает значения в интервале $\lambda_{1} \in\left[\lambda-\lambda_{*}, \lambda+\lambda_{*}\right]$, поэтому амплитуда $A$ может быть как больше, так и меньше значения, полученного при $\varepsilon=0$. Разумеется, при $\lambda<\lambda_{*}$ вопрос о максимальной амплитуде теряет смысл, так как в этом случае система динамически неустойчива.

Таким образом, выявленные выше условия асимптотической устойчивости положения равновесия остаются справедливыми и при вынужденных колебаниях, однако в этом случае они свидетельствуют не о затухании колебаний, а об асимптотическом приближении возмущенного движения к исследуемому режиму.

При анализе параметрических резонансов в многомассовых системах можно воспользоваться переходом к квазинормальным координатам при усредненных значениях коэффициентов форм [10, 13]. Разумеется, различные формы колебаний реономных систем между собой связаны, что приводит к некоторой погрешности расчета. Кроме того, «усредняя» формы колебаний, мы тем самым исключаем инерционные составляющие, возникающие при их изменении. Однако обычно связанность системы в рассматриваемом классе задач оказывается довольно слабой. К тому же принудительное разделение форм в основных зонах параметрического возбуждения, как правило, несколько завышает запас устойчивости.

\section{3.Колебания привода цикловой машины при учете динамической характеристики электродвигателя}

Динамическая характеристика электродвигателя. Выше при динамическом анализе механизмов мы принимали, что угловая скорость двигателя является постоянной или известной функцией времени. Однако такой подход нуждается в проверке, а иногда и в некоторой корректировке результатов. Динамическая модель исполнительной машины, строго говоря, должна быть дополнена учетом динамической характеристики источника энергии (двигателя), что позволяет определить неравномерность вращения ведущих звеньев и связанную с этим виброактивность привода. В некоторых автоматизированных приводах и робототехнических устройствах следует также учитывать системы управления движением [3, 16, 19]. В этом параграфе мы ограничимся вопросами, которые имеют непосредственное отношение к динамике цикловых технологических машин, в которых управление в силу 
жестких ограничений определяется циклограммой программного движения рабочих органов.

Электродвигатели относятся к электромеханическим системам, в которых динамические процессы характеризуются взаимодействием двух форм движения - электрической и механической. Описание электромагнитных колебательных процессов в двигателях связано с решением достаточно сложных систем; нелинейных дифференциальных уравнений. Однако применительно к установившимся режимам в инженерной практике хорошо зарекомендовали себя приближенные линеаризованные уравнения, называемые динамическими характеристиками двигателя, которые для двигателей постоянного тока и асинхронных двигателей могут быть представлены как [3, 10, 16, 22]

$$
\omega_{\ddot{A}}=\omega_{\ddot{A}}^{0}\left[1-v_{\ddot{A}}\left(M_{\ddot{A}}+T_{\ddot{A}} \grave{I}_{\ddot{A}}\right)\right] \text {, }
$$

где $\omega_{\ddot{\AA}}, \omega_{\ddot{A}}^{0}-$ угловые скорости электродвигателя и идеального холостого хода; $v_{\AA}-$ коэффициент крутизны статической характеристики; і̀ теля; $\grave{\AA}_{\check{\AA}}$ - электромагнитная постоянная времени двигателя, зависящая от параметров его электрической цепи.

Для асинхронных электродвигателей $v_{\text {̆ }}$ и $T_{\text {д }}$ определяются по следующим приближенным зависимостям: $\grave{O}_{\ddot{A}}=1 /\left(2 \pi f_{\tilde{\mathrm{N}}} S_{\hat{\mathrm{E}}}\right) ; v_{\tilde{A}}=s_{\hat{\mathrm{E}}} /(2 \grave{I}$ i $\xi)$,где $s_{\mathrm{K}}=\left(1-\omega_{\AA}^{\mathrm{i}} / \omega_{\AA}^{0}\right)\left(\xi+\sqrt{\xi^{2}+1}\right)-$ критическое скольжение; $M_{\mathrm{H}}, \omega_{\AA}^{1}-$ номинальные момент и угловая скорость двигателя; $f_{\mathrm{C}}$ - частота сети, Гц; $\xi$ - отношение максимального момента к номинальному.

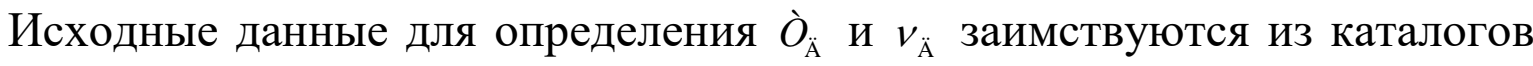
электродвигателей. Если в уравнении (5.11) принять $\grave{O}_{\AA}=0$, то характеристика называется статической.

Случай $\boldsymbol{J}=$ const. Если приведенный к валу двигателя момент инерции $J$ является постоянным, то

$$
J \dot{\omega}_{\ddot{\AA}}=\grave{I}_{\ddot{\mathrm{A}}}-\grave{I}_{\tilde{\mathrm{N}}},
$$

где $\grave{I}_{\tilde{\mathrm{N}}}-$ приведенный к валу двигателя момент сопротивления. Отсюда сле-

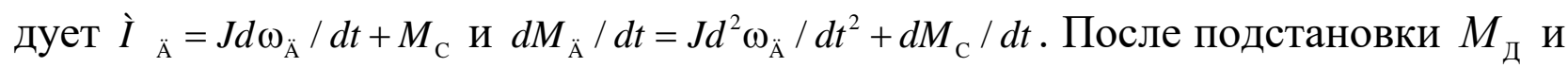
$d M_{\text {д }} / d t$ в (5.12) получаем

$$
v_{\ddot{\mathrm{A}}} T_{\ddot{\mathrm{A}}} J \ddot{\omega}_{\ddot{\mathrm{A}}}+v_{\ddot{\mathrm{A}}} J \dot{\omega}_{\ddot{\mathrm{A}}}+\omega_{\ddot{\mathrm{A}}} / \omega_{\ddot{\mathrm{A}}}^{0}=1-v_{\ddot{\mathrm{A}}}\left(\grave{I}_{\tilde{\mathrm{N}}}+\grave{O}_{\ddot{\mathrm{A}}} \grave{I}_{\tilde{\mathrm{N}}}\right) \text {. }
$$

Далее представим момент сопротивления $\grave{I}_{\tilde{\mathrm{N}}}$ в виде суммы среднего значения $\overleftarrow{I}_{\text {с }}$ и переменной составляющей $\Delta M_{\mathrm{C}}(t)$. Соответственно функцию 
$\omega_{\ddot{\AA}}(t)$ также представим как $\omega_{\ddot{\AA}}(t)=\bar{\omega}_{\ddot{\AA}}+\Delta \omega_{\ddot{\AA}}(t)$. Постоянная составляющая $\bar{\omega}_{\ddot{\AA}}$ определяется статической характеристикой двигателя:

$$
\bar{\omega}_{\ddot{\AA}}=\omega_{\tilde{\AA}}^{0}\left(1-v_{\tilde{\AA}} I_{\tilde{\mathrm{N}}}^{-}\right) \text {. }
$$

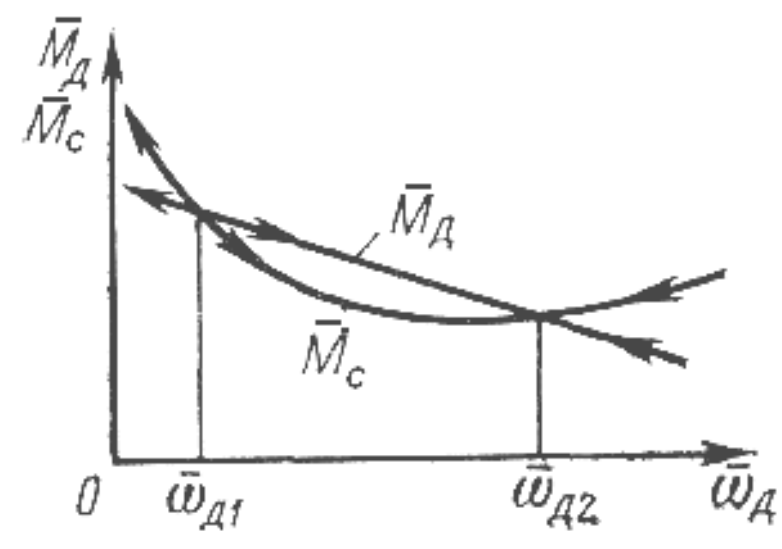

Рис. 5.3. Графики $\bar{M}_{\text {à }} \bar{M}_{\mathrm{C}}$

В инженерной практике возникают ситуации, когда определение $\bar{\omega}_{\AA}$ требует некоторых уточнений. Пусть, например, графики $\bar{M}_{\ddot{A}}$ и $\bar{M}_{C}$ имеют вид, показанный на рис.5.3. Точки пересечения кривых соответствуют двум стационарным режимам. Однако при угловой скорости $\bar{\omega}_{\ddot{A} 1}$ режим будет неустойчивым, так как любое отклонение от него приводит к возникновению дестабилизирующего момента. Устойчивому стационарному режиму отвечает значение $\omega_{\ddot{A} 2}$.

При учете (5.14)перепишем уравнение (5.13)в следующем виде:

$$
\Delta \ddot{\omega}_{\ddot{\mathrm{A}}}+2 n \Delta \dot{\omega}_{\ddot{\mathrm{A}}}+k^{2} \omega_{\ddot{\mathrm{A}}}=W(t),
$$

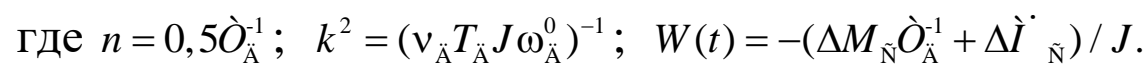

Итак, нахождение переменной составляющей угловой скорости двигателя $\Delta \omega$, определяющей неравномерность вращения входного звена машинного агрегата, снова свелось к решению линейного неоднородного дифференциального уравнения второго порядка, подробно исследованного в данной главе. Заметим однако, что в отличие от ранее рассмотренных случаев, когда диссипативная сила была вызвана в основном конструкционным демпфированием, параметр $n$ здесь может оказаться соизмерим с $k$. Поэтому при расчете собственной частоты $k_{1}$ следует пользоваться зависимостью

$$
k_{1}=\sqrt{k^{2}-n^{2}}=\sqrt{\left(J v_{\ddot{\AA}} T_{\ddot{\AA}} \omega_{\AA}^{0}\right)^{-1}-0,25 T_{\AA}^{-2}} .
$$

При $T_{\AA}=T_{\AA}^{*}=0,25 v_{\AA} \omega_{\AA}^{0} J$ собственная частота обращается в нуль. Таким образом, при $T_{\text {д }} \leq T_{\text {д }}^{*}$ решение однородного дифференциального уравнения, соответствующего (5.15), становится апериодическим. С увеличением $T_{\AA}$ до значения $T_{\AA}^{* *}=0,5 v_{\AA} \omega_{\AA}^{0} J$ величина $k_{1}$ растет до своего максимального значения 
$k_{1 \max }=\left(v_{\AA} \omega_{\AA}^{0} J\right)^{-1}$; при $T_{\ddot{\AA}}>T_{\AA}^{* *}$ собственная частота с ростом электромагнитной постоянной времени $T_{\check{\AA}}$ соответственно убывает. Такое влияние параметра $T_{\text {д }}$ связано с тем, что динамическая характеристика двигателя соответствует модели, в которой ротор соединен со статором посредством «упругого элемента» с коэффициентом жесткости $c_{\ddot{\AA}}=\left(v_{\AA} \omega_{\AA}^{0} T_{\AA}\right)^{-1}$ и последовательно включенного демпфера, развивающего момент сопротивления $-b_{\ddot{\AA}} \Delta \dot{\omega}_{\ddot{A}} \quad$ при $b_{\ddot{A}}=\left(v_{\ddot{\AA}} \omega_{\ddot{A}}^{0}\right)^{-1}$. При малых значениях $T_{\text {д }}$ определяющую роль играет демпфер, что и приводит к возможности появления апериодических решений; при больших значениях $T_{\AA}$ «жесткость» убывает, и соответственно уменьшается собственная частота.

Как показывает анализ, характеристика асинхронного двигателя и двигателя постоянного тока эквивалентна податливости упругого элемента, которая обычно существенно превосходит упругие элементы привода. Это позволяет при изучении динамики машинного агрегата исследовать неравномерность вращения вала двигателя с помощью сравнительно простых моделей, считая в первом приближении остальную кинематическую цепь либо абсолютно жесткой, либо ограничиваясь учетом наиболее податливых элементов привода.

Одним из традиционных способов уменьшения неравномерности вращения входных звеньев приводов машин является увеличение момента инерции $J$ за счет установки маховика. Однако, как показывает анализ, увеличение $J$ приводит к уменьшению $\Delta \omega_{\AA}$ только в зарезонансном режиме, когда частота вынуждающего момента $\omega>k_{1}$. Если при этом с целью отстройки от резонанса принять $\omega \geq \sqrt{2} k_{1}$, то при установке маховика по крайней мере должно быть

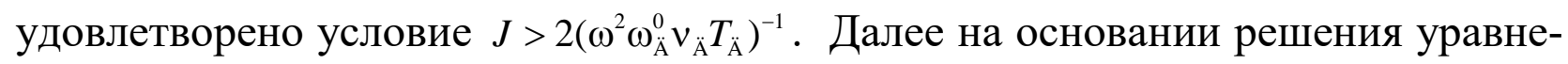
ния (5.15) может быть определен коэффициент неравномерности $\delta_{\mathrm{I}} \approx 2\left|\Delta \omega_{\ddot{\AA}}\right|_{\max } / \bar{\omega}_{\ddot{\AA}}$, где $\bar{\omega}_{\ddot{\AA}}-$ среднее значение угловой скорости двигателя.

Следует, однако, иметь в виду, что существенное увеличение момента инерции $J$ не является желательным для переходных режимов, поскольку при этом возрастают динамические нагрузки при разбеге машины.

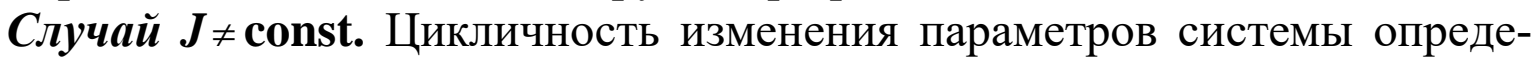
ляется средней угловой скоростью главного вала $\bar{\omega}$. При этом вместо зависимости (5.118) следует записать

$$
J(\varphi) \dot{\omega}_{\ddot{a}}+0,5 \omega_{\ddot{a}}^{2} \frac{d J}{d \varphi}(\varphi)=M_{\text {ă }}\left(\omega_{\ddot{a}}\right)-M_{c}(\omega, \varphi) .
$$

Здесь $\varphi-$ угол поворота главного вала.

Тогда, снова, представляя угловую скорость двигателя в виде суммы постоянной и переменной составляющих, после линеаризации в окрестности программного движения получаем

$$
\Delta \ddot{\omega}_{\ddot{a}}+2 n\left(\varphi_{*}\right) \Delta \dot{\omega}_{\ddot{a}}+k^{2}\left(\varphi_{*}\right) \Delta \omega_{\ddot{a}}=W\left(\varphi_{*}\right),
$$

где $2 n=T_{\text {ă }}^{-1}+2 J^{\prime} \bar{\omega} / J ; k^{2}=J^{-1}\left[\left(v_{\text {ă }} T_{\ddot{a}} \bar{\omega}_{\ddot{a}}\right)^{-1}+\bar{\omega} J^{\prime} T_{\text {ă }}^{-1}\right] ; W=-J\left(\Delta M T_{\text {ă }}^{-1}+\Delta M^{\prime} \bar{\omega}+0,5 \bar{\omega}^{2} u J^{\prime} T_{\text {ă }}^{-1}\right)$; 
$u=\omega_{\text {ä }} / \omega$ - передаточное число ; $\Delta M=M-\bar{M} ;()^{\prime}=d / d \varphi$; чёрточка соответствует среднему значению на периоде $\tau=2 \pi / \omega$.

Таким образом, относительно $\Delta \omega_{a}$ мы снова получили линейное дифференциальное уравнение второго порядка с переменными коэффициентами. «Собственная» частота согласно (5.6) равна $p=\sqrt{k^{2}-n^{2}-\dot{n}}$. Специфика данной модели состоит в относительно большом значении диссипативного члена, что практически исключает возможность параметрического резонанса на этой частоте. Кроме того, это может привести к большой разнице между $k$ и $p$ и даже к переходу в апериодический режим. Отметим, что нередко достаточную точность даёт решение при усреднённом значении $J \approx \bar{J}=$ const (см. выше). Более подробно аналитическое решение колебательных режимов при учёте характеристик электродвигателя и переменном приведённом моменте инерции анализируется в работах [3, 16,19]

\section{4. Искажения программного движения в цикловых колебательных системах, вызванные двигателем}

В ряде машин программное движение совершает массивный исполнительный орган, который, по существу, определяет динамическую нагрузку электродвигателя. Такая ситуация имеет место в приводе стола плоскопечатной машины, приводе кареток и других массивных исполнительных органов текстильных машин, в автоматах и автоматических линиях в самых различных отраслях промышленности. В подобных случаях динамические процессы, происходящие в двигателе, проявляются не только в изменении силовых характеристик системы, но и в динамических ошибках заданного программного движения $[12,23]$. В качестве примера на рис.6.4 приведена осциллограмма кинематических характеристик выходного звена кулачкового механизма при симметричном законе программного движения. На разбеге толкателя из-за больших инерционных нагрузок угловая скорость двигателя существенно снизилась, что привело к значительным ускорениям на выбеге и конечном итоге к виброударному режиму на участке теоретического выстоя .

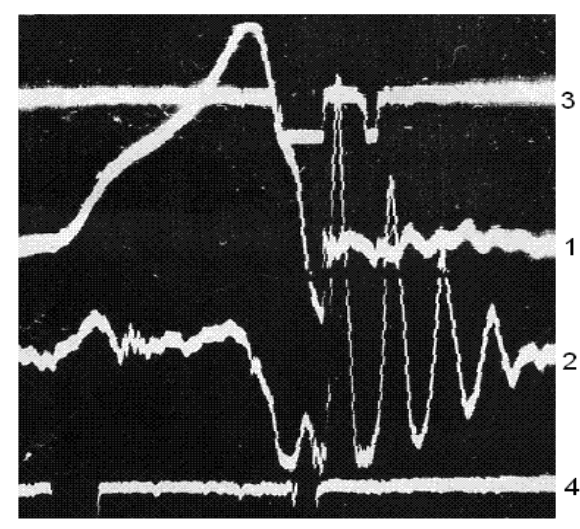

Рис. 5.4. Искажения характеристик программного движения: 1- скорость; 2 - ускорения; 3- участки разрыва кинематического контакта; 4 - отметки начала и конца программного движения

Рассмотрим динамическую модель (рис. 5.5), в которой учтены характеристики двигателя, привода и колебательная система выходного звена. 


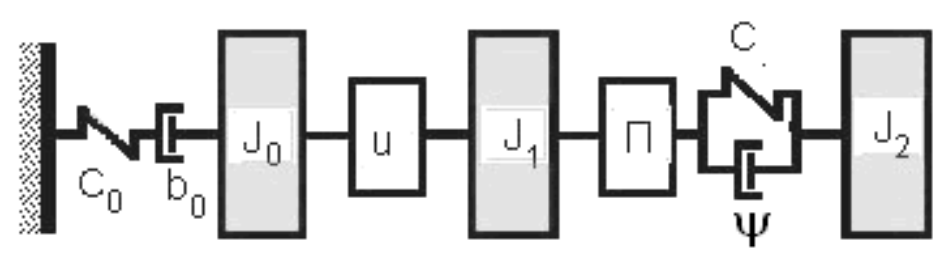

Рис. 5.5. Динамическая модель цикловой машины с односторонним приводом

Для выявления искажений заданного программного движения сначала рассмотрим частный случай при абсолютно жёстком механизме $(c \rightarrow \infty)$. На рис.5.6 приведены графики функции $w=-\ddot{q}\left(\varphi_{*}\right)$, которые пропорциональны моменту инерционных сил выходного звена. При этом кривая 1 отвечает исходному гармоническому закону изменения ускорений, а кривые 2, 3 - учёту соответственно статической и динамической характеристик электродвигателя.

Из анализа графиков следует, что наиболее значительные искажения имеют место при учёте статической характеристики. Это связано с появлением второй гармоники в моменте инерционных сил, приведённом к валу двигателя, который пропорционален П'П" (см.п.1.3). При учёте динамической характеристики этот эффект за счёт электромагнитной постоянной времени смягчается, однако по сравнению с исходными ускорениями возрастают экстремальные значения.

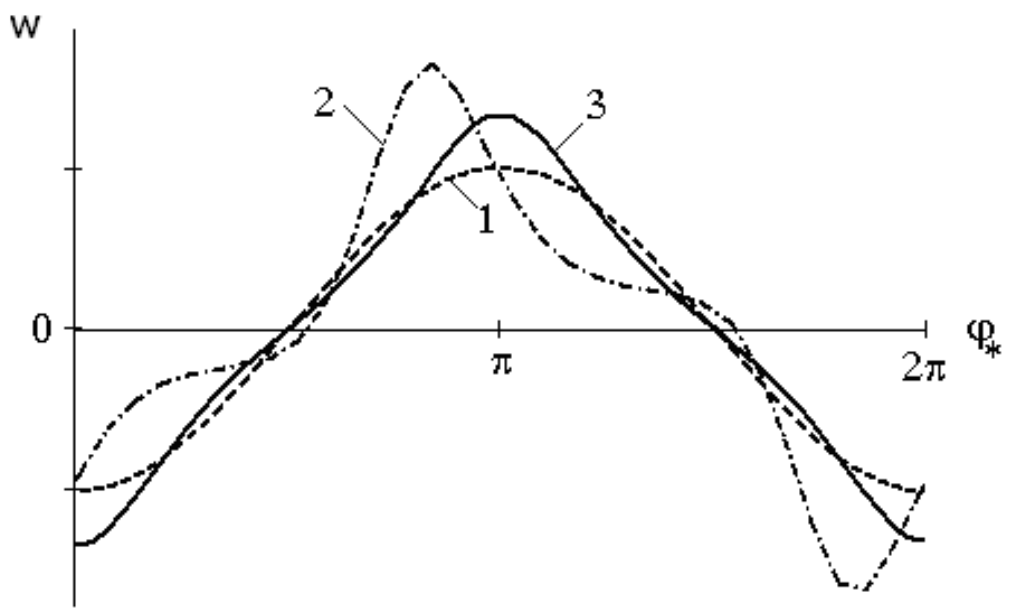

Рис. 5.6. Искажения ускорений программного движения, связанные с влиянием двигателя

Представляет интерес, насколько высокочастотные колебания, возбуждаемые на выходном звене, искажаются при совместном учёте переменности параметров, зазоров и влияния двигателя. Обратимся к более сложной динамической модели с двухсторонним приводом при учете характеристик электродвигателя, нелинейной функции положения и зазоров (рис. 5.7). На рис. 5.8. для этой модели приведены результаты компьютерного моделирования. Рассмотрим два случая: при отсутствии силового замыкания $\left(c_{0}=0\right.$, рис.5.8,a) и с силовым замыканием, исключающим возможность соударений в зазоре $\left(c_{0} \neq 0\right.$, рис. $\left.5.8, \sigma\right)$. 


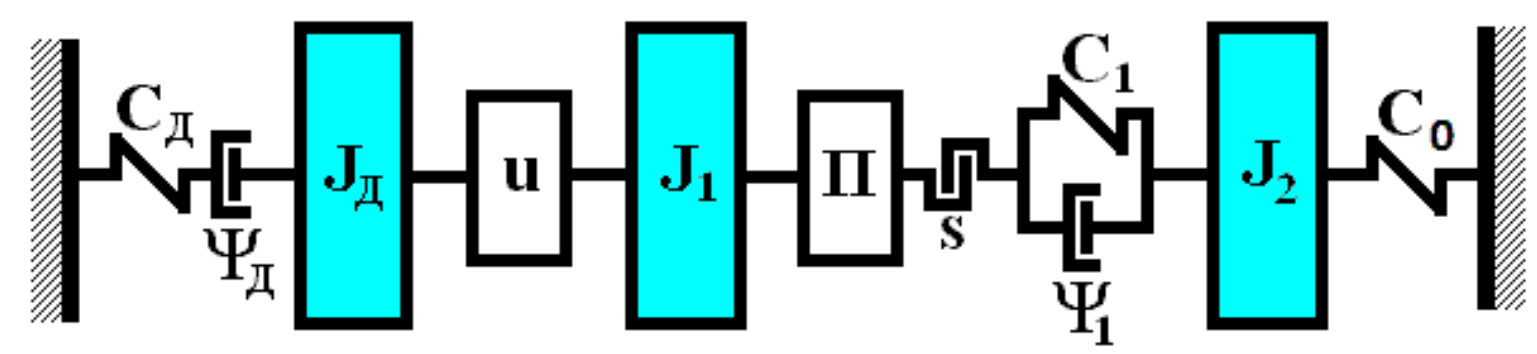

Рис. 5.7. Динамическая модель цикловой машины с двухсторонним приводом: $u$ - передаточное отношение; $c_{0}$ - коэффициент жесткости замыкающей системы

Кривая $\omega$ соответствует исходному гармоническому закону программного движения, искаженному из-за неравномерного вращения ротора двигателя. Кривые $\Delta \omega_{1}$ и $\Delta \omega_{2}$, отвечающие динамической ошибке угловой скорости электродвигателя, различаются тем, что во втором случае дополнительно учтены фазовые сдвиги угла поворота главного вала. При отсутствии силового замыкания в зоне перехода через зазор (см. гл.4) возникает импульсное возбуждение, вызывающее высокочастотные колебания выходного звена и существенные искажения заданного закона изменения ускорений ведомого звена (кривая $\varepsilon$ ). Нередко это приводит к неприемлемым режимам работы.

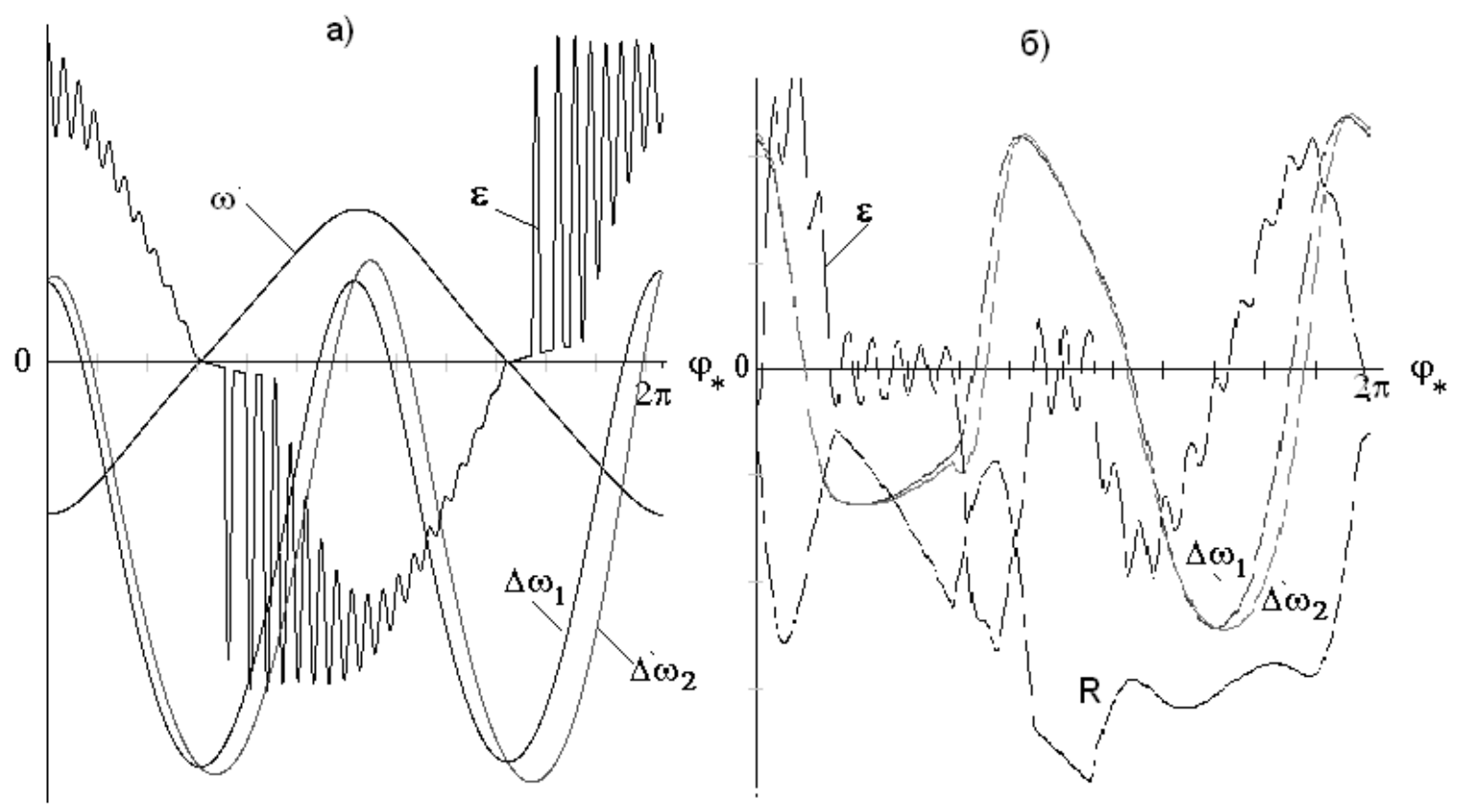

Рис.5.8. Результаты компьютерного моделирования привода цикловой машины при учете влияния двигателя и зазоров

При правильном выборе условий силового замыкания (см. гл. 4) реакция со стороны ведомого звена на привод (кривая $R$ ) на всем кинематическом цикле не меняет своего знака, что исключает возможность ударного возбуждения колебаний в зазоре. 
Анализ графиков позволяет сделать следующие выводы:

1. Ввиду существенной разницы в парциальных частотах электродвигателя и механической системы высокочастотные колебания выходного звена, как правило, не находят отражения в угловой скорости двигателя.

2. Низкочастотные колебания выходного звена, обусловленные программным движением (кривая $\omega$ ), могут привести к значительной неравномерности вращения главного вала (кривые $\left.\Delta \omega_{1}, \Delta \omega_{2}\right)$. При этом не только изменяются экстремальные значения кинематических характеристик программного движения, но и отрезки времени, отвечающие переходным участкам изменения программных ускорений. Это, в свою очередь, проявляется на уровне возбуждаемых колебаний выходного звена.

3. Фазовые сдвиги по углу ф могут существенно исказить заданные программным движением длительность участков разбега и выбега, что приводит к нарушениям точности воспроизведения кинематических характеристик выходного звена. Это особенно важно в цикловых механизмах с силовым замыканием, когда могут возникнуть разрывы кинематического контакта и виброударные режимы.

4. Силовое замыкание устраняет высокочастотные колебания, возникающие при выборке зазоров, однако может повлиять на искажения программного движения из-за неравномерности вращения ротора двигателя. 


\section{ГЛАВА 6. КОЛЕБАНИЯ КОМПЕНСАТОРА С ПРОГРАММНЫМ УПРАВЛЕНИЕМ НАТЯЖЕНИЯ ГИБКИХ СВЯЗЕЙ}

\section{1 Динамическая модель}

Для силового замыкания механизмов реализующих периодическое движение рабочих органов нередко используются устройства с гибкими связям. Однако в некоторых машинах эти устройства выполняют более сложные функции, непосредственно связанные с выполняемым технологическим процессом. Динамика механизмов с гибкими связями представляет большой интерес при проектировании текстильных, полиграфических, швейных, бумагоделательных машин, а также приводов с ременными передачами машин различного назначения.

Для стабилизации движения гибкой ленты или нити широко используются различного рода натяжные устройств, основное назначение которых обычно состоит в поддержании квазипостоянного натяжения и в подавлении относительно малых возмущений. Эти устройства, называемые стабилизаторами или компенсаторами натяжения, представляют собой ролик или палец, имеющий контакт с гибким элементом, а также с корпусом посредством линейного или нелинейного упругого элемента. Наряду с отмеченной функцией натяжных устройств, возможны случаи, когда палец натяжного устройства совершает программно заданные большие перемещения, причём в некоторых случаях технологический процесс предусматривает даже запрограммированный разрыв кинематического контакта с гибкой связью. Такая ситуация, в частности, возникает в нитепритягивателях швейных и обувных машин, когда на определённом временном интервале технологического цикла нить должна быть свободной, т.е. натяжение должно отсутствовать. Отмеченные факторы существенно проявляются на динамике подобных устройств

На рис.6.1,а приведена динамическая модель пружинного компенсатора натяжения гибкой связи. Приняты следующие условные обозначения: $c_{i}-$ коэффициенты жёсткости, $\psi_{i}$ приведённые коэффициенты рассеяния, $m$ - масса.

a)

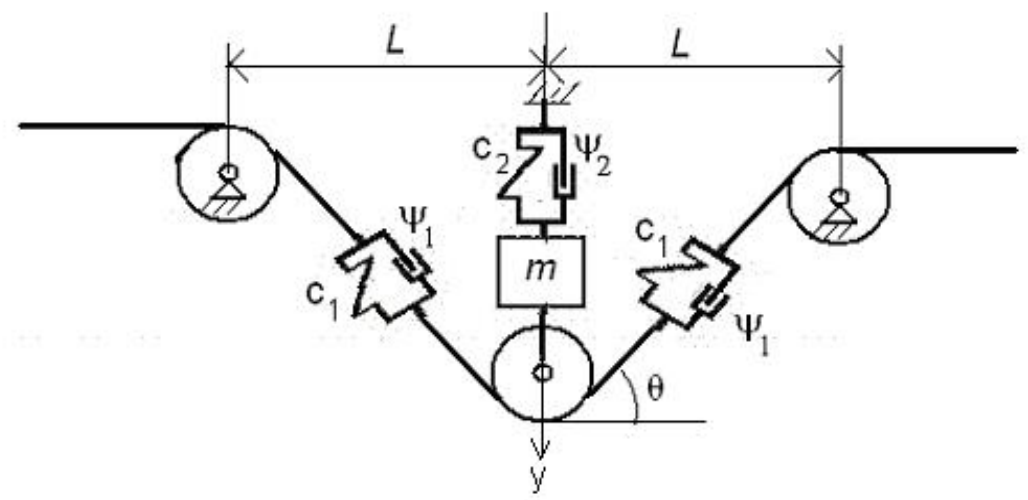

б)

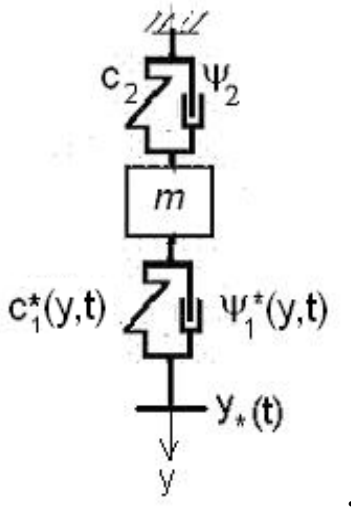

Рис. 6.1. Динамическая модель 
Для коэффициентов $c_{1}$ и $\psi_{1}$ могут быть использованы приведённые значения, учитывающие упругие и диссипативные свойства нити за пределами двух ветвей. Предполагается, что механический привод достаточно массивен по сравнению с натяжным устройством, поэтому его колебания слабо зависят от колебаний массы $m$. В то же время обратное влияние может быть существенным. В данной статье исследуются динамические эффекты, возникающие при программном изменении длины ветви нити $l(t)=L / \cos \theta(t)$. Как уже отмечалось, нередко возникает необходимость в «свободной» (не натянутой) нити. В подобных случаях предельные перемещения компенсатора должны быть ограничены соответствующими упорами.

Осуществим переход к модели, показанной на рис.6.1,б. Потенциальная энергия при деформации одной ветви равна $P^{2} /\left(2 c_{1}\right)$, где $P$ - сила натяжения. Сила $P$ и реакция нити на ролик $R$ связаны следующей очевидной зависимостью: $R=2 P \sin \theta$. На основании условия баланса потенциальной энергии имеем $c_{1}^{*}=2 c_{1} \sin ^{2} \theta$. Аналогичная зависимость связывает коэффициенты рассеяния: $\psi_{1}^{*}=2 \psi_{1} \sin ^{2} \theta$. Приведённый коэффициент жёсткости системы равен $c=2 c_{1} \sin ^{2} \theta+c_{2}$, а приведённый коэффициент рассеяния $\psi=2 \psi_{1}^{*} c_{1}^{*} / c+\psi_{2} c_{2} / c$ $[10,13]$.

В качестве безразмерной обобщённой координаты примем $q=\operatorname{tg} \theta=\mathrm{y} / \mathrm{L}$. Тогда $\sin ^{2} \theta=q^{2} /\left(1+q^{2}\right)$ и $c=c_{2}\left[1+2 \zeta^{2} q^{2} /\left(1+q^{2}\right)\right]$, где $\zeta^{2}=c_{1} / c_{2}$. Потребуем, чтобы при $\theta=\theta_{0}$ имело бы место статическое равновесие системы. Для определения внешней силы $F_{0}$, которую необходимо приложить к системе при $\theta=0$, составим уравнение статического равновесия:

$$
c_{2} y+F_{0}=2 \zeta^{2} c_{2} L \int_{0}^{q} q^{2} /\left(1+q^{2}\right) d q
$$

Здесь левая часть равенства отвечает реакции $F_{1}$ со стороны пружины компенсатора, а правая часть - реакции $R$ со стороны нити. После интегрирования правой части (6.1) получаем

$$
w=\left(2 \zeta^{2}+1\right) \operatorname{tg} \theta_{0}-2 \zeta^{2} \theta_{0},
$$

где $\quad w=F_{1}\left(\theta_{0}\right) /\left(c_{2} L\right)$.

Далее рассмотрим источники возбуждения колебаний. Пусть $l=l_{0} f(t)$, где $f(t)=1+\Delta f(t)$. Принимая во внимание, что $y / l=\sin \theta$, имеем $\theta(t)=\arcsin \left(f^{-1}(t) \sin \theta_{0}\right)$. Можно показать, что при этом на систему действует кинематическое возбуждение, которое в безразмерной форме с достаточной точностью описывается как $y^{*}(t)=2 c_{2} \zeta^{2} \sin ^{3} \theta_{0}\left[1-f^{-1}(t)\right]$. Пусть движение массы $m$ ограничено двумя упорами, расположению которых отвечают координаты $q_{1}$ (верхний упор ) и $q_{2}$ (нижний упор (см. рис.6.1,б). Тогда при $q_{1}<q<q_{2}$ на основании (6.1), (6.2) система описывается следующим нелинейным дифференциальным уравнением: 


$$
\ddot{q}+2 \delta k \dot{q} \sqrt{\left(1+2 \zeta_{*}^{2} \sin ^{2} \theta_{0}\right)}+k^{2}\left[q\left(1+2 \zeta_{*}^{2}(q-\operatorname{arctg} q)\right]=k^{2}\left(w_{*}+\Delta w(t)\right) .\right.
$$

Здесь $\Delta w(t)=2 \zeta_{*}^{2}\left[\operatorname{tg}\left(\theta_{0}+\Delta \theta\right)-\operatorname{tg} \theta_{0}+\Delta \theta\right] ; \eta_{1}\left(q-q_{1}\right), \eta_{2}\left(q_{2}-q\right) ; \eta_{i}-$ единичные функции, управляющие «подключением» $c_{1} ; \delta=\psi /(4 \pi) ; k=\sqrt{c_{2} / m}$;

Таким образом, при $q<q_{1}$ и абсолютно жёстких упорах $\left(c=c_{2} \rightarrow \infty\right.$, следовательно, $\zeta \rightarrow 0$ и $c \rightarrow \infty$, а при $q>q_{2}$ имеем $c=c_{1} \rightarrow \infty$. В последнем случае натяжение гибкого элемента обращается в нуль.

\section{2. Условия устойчивости при совместном кинематическом и параметрическом возбуждении}

Гармоническая пульсация частоты. Так как положение «статического» равновесия оказывается функцией времени, то спектр «собственных» частот также зависит от времени, а, следовательно, наряду с кинематическим возбуждением возможно и параметрическое. При этом возможны существенные коррективы диссипативных характеристик [10,13]. В частности, в [10] было исследовано дифференциальное уравнение

$$
\ddot{q}+2 n_{0} \dot{q}+k_{0}^{2}(1-\varepsilon \sin p t) q=a \omega^{2} \sin \left(\omega t-\gamma_{1}\right),
$$

где $k_{0}$ - усреднённая частота свободных колебаний, частота параметрического возбуждения

На основании условий энергетического баланса, принимая $\omega=2 k_{0}$, $q=A \sin \left(\omega t-\gamma_{2}\right)$, можно показать, что возможен установившийся режим с амплитудой

$$
A=0,5 \omega^{2} k_{0}^{-2} a \sin \left(\gamma_{2}-\gamma_{1}\right) /\left(\delta-\delta_{*} \cos 2 \gamma_{2}\right) .
$$

Здесь $\delta=n_{0} / k_{0}=\psi /(4 \pi) ; \quad \delta_{*}=0,25 \varepsilon ; \quad \gamma_{2}-\gamma_{1}=\pi / 2-$ фазовый сдвиг, соответствующий резонансу.

Из формулы (6.4) следует, что амплитуда А в первом приближении отвечает некоторой условной системе при отсутствии параметрического возбуждения, но с изменённым уровнем диссипации $\delta-\delta_{*} \cos 2 \gamma_{2}$. При $\delta<\delta_{*} \cos 2 \gamma_{2}$ в рамках линейной постановки задачи установившийся режим не возможен $(A \rightarrow \infty)$. Представив в виде усечённого ряда Маклорена функцию $\operatorname{arctg} y \approx y-y^{3} / 3$ и временно исключив из рассмотрения влияние упругих упоров, приведём уравнение (6.5) к виду уравнения Дуффинга

$$
\ddot{q}+2 \delta p_{0} \dot{q}+\Psi(q)=[w+\Delta w(t)] k^{2},
$$

где $\Psi(q)=k^{2}\left(q+2 \zeta_{*}^{2} q^{3} / 3\right) ; p_{0}=\sqrt{1+2 \zeta_{*}^{2} \sin ^{2} \theta_{0}}-$ «собственная» частота в положении равновесия. 
Принимая решение в виде $q=A_{0}+A \sin \omega t$, с помощью метода гармонической линеаризации запишем линеаризованное дифференциальное уравнение в виде

$$
\ddot{q}+2 \delta p_{0} \dot{q}+p^{2}\left(A_{0}, A\right) q+\Psi_{0}\left(A_{0}, A\right)=W_{0}+W_{1} \sin (\omega t+\gamma) .
$$

С помощью метода гармонической линеаризации получаем

$$
A_{0}^{2}(t)=2 /\left[3 \zeta_{*}^{2} \operatorname{tg}^{2}\left(\theta_{0}+\Delta \theta(t)\right)\right]
$$

При $\quad A \rightarrow 0$ имеем $p^{2}\left(A_{0}, t\right)=k^{2}\left\{1+2 \zeta_{*}^{2} \operatorname{tg}^{2}\left[\arcsin \left(f^{-1}(t) \sin \theta_{0}\right)\right]\right\}$. При $f(t)=1-a \sin \omega t$ отсюда следует $\Delta p^{2}=p_{1}^{2}-p_{0}^{2}=2 k^{2} \zeta_{*}^{2}\left[\sin ^{2} \theta_{0} /\left(f^{2}-\sin ^{2} \theta_{0}\right)-\operatorname{tg}^{2} \theta_{0}\right]$, где $p_{1}^{2}, p_{0}^{2}-$ значения функции $p^{2}$ при $f=1-a$ и $f=1$. Тогда глубина пульсации параметрического возбуждения $\varepsilon$ и максимальное критическое значение коэффициента демпфирования $\delta_{*}$ определяются как

$$
\varepsilon=\zeta^{2} a^{2} /\left(1+\zeta^{2} a^{2}+2 \zeta^{2} \operatorname{tg}^{2} \theta_{0}\right) ; \quad \delta_{*}=0,5 \varepsilon
$$

На основании (6.6) построены графики $\delta_{*}(a, \zeta)$ (рис.2) при $\theta_{0}=0,3$ и $\delta_{*}\left(\theta_{0}, \zeta\right)$ при $a=0,2$. При $\theta_{0} \rightarrow \pi / 2$ имеем $\delta_{*} \rightarrow 0$, и параметрическое возбуждение отсутствует.
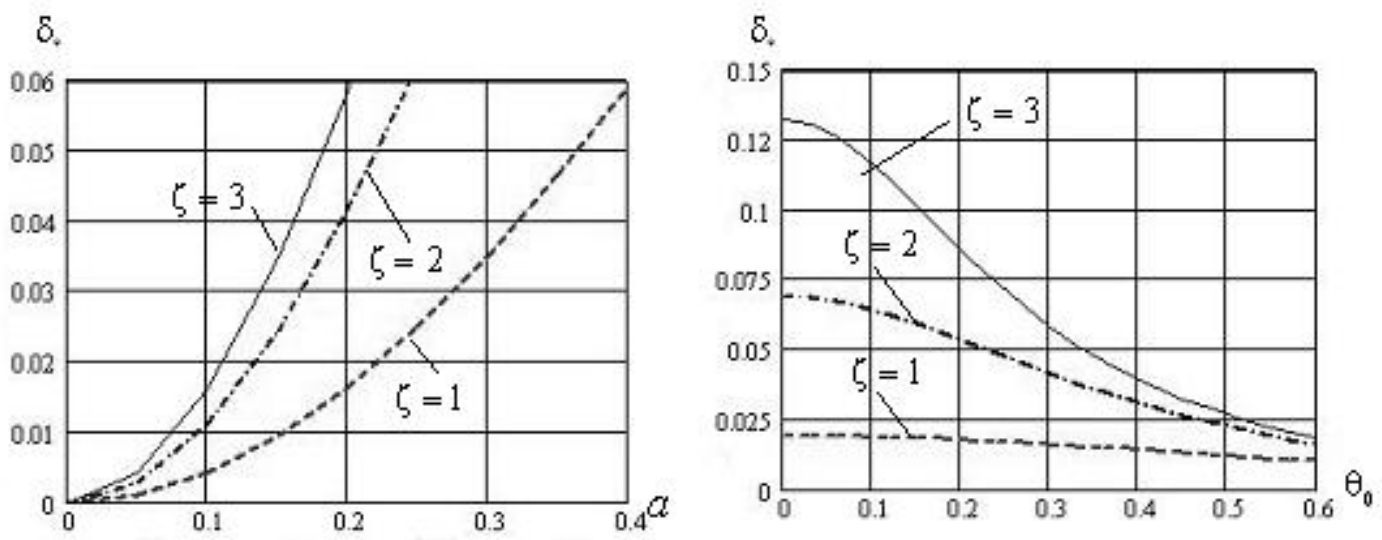

Рис. 6.2. Графики критических значений коэффициентов демпфирования

Амплитуда резонансных вынужденных колебаний с учётом корректив, вызванных параметрическим возбуждением, может быть оценена следующим образом:

$$
A_{*}=a \zeta^{2} \sin ^{3} \theta_{0}\left(1+2 \zeta^{2} \sin ^{3} \theta_{0}\right)^{-1}\left[\delta-\delta_{*}\left(\zeta, \theta_{0}, a\right)\right]^{-1}
$$

При $\delta \leq \delta_{*}$ формально $A_{*} \rightarrow \infty$, однако за счёт нелинейных факторов происходит срыв колебаний, поэтому неограниченное возрастание амплитуд не 
реализуется. Можно показать, что амплитуда параметрических колебаний при учёте нелинейности в нашем случае определяется как

$$
A_{\varepsilon}=\sqrt{2 \zeta^{-2}\left(\lambda^{2}-1\right) \pm \sqrt{\left(0,25 \varepsilon^{2}-\lambda^{2} \delta^{2}\right) /\left(1+2 \zeta^{2} \sin ^{2} \theta_{0}\right)^{2}}},
$$

где $\lambda=\omega /\left(k \sqrt{1+2 \zeta^{2} \sin \theta_{0}}\right)$.

Графики функции $A_{\varepsilon}(z, \varsigma)$ для $a=0,5$ приведены на рис. 6.3. Штриховыми линиями показаны неустойчивые ветви амплитудно-частотной характеристики. При $0,5 \varepsilon=\lambda \delta$ наступает срыв колебаний.

С целью проверки эффективности полученных выше аналитических оценок проведён численный эксперимент на базе нелинейного дифференциального уравнения (6.5). Линии 1 и 2 на графике $q(t)$ отвечают положению упоров, а прямая 3 - положению равновесия, определённого по условию (6.1).

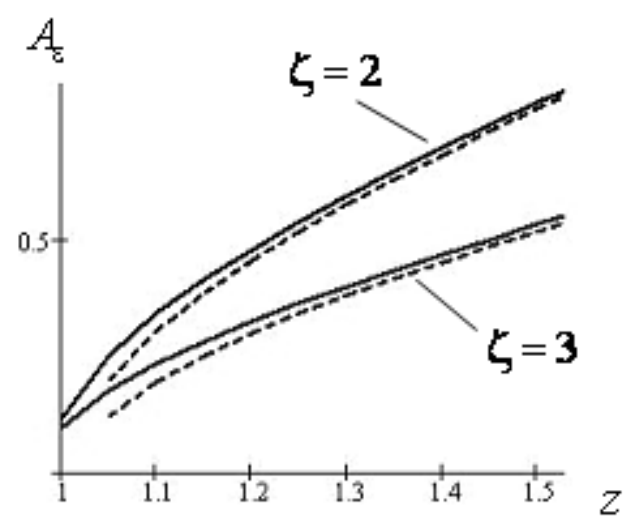

Рис. 6.3. Фрагменты амплитудно-частотных характеристик

На рис.6.4 приведены характерные режимы $\quad\left(k=2 \mathrm{c}^{-1}, \theta_{0}=0,3, \zeta=3\right)$, возникающие при совместном кинематическом и параметрическом возбуждении. При компьютерном моделировании была учтена жёсткость упоров.

Peжимы 1,2 $\left(\omega=3,2 \mathrm{c}^{-1}\right)$. Система находится в окрестности «собственной» частоты (см.выше), однако, из-за нелинейной характеристики в частотном спектре наиболее значительная пульсация имеет место на частоте $2 \omega$. Следовательно, $p \approx \omega$, что одновременно отвечает зоне главного параметрического резонанса. О параметрической природе возбуждения свидетельствует наблюдаемый на графиках экспоненциальный характер нарастания колебаний, сопровождающийся в режиме 1 срывом колебаний. При подключении упоров (режим 2) дополнительное возбуждение и рост виброактивности, как уже отмечалось, связаны с возникновением виброударных режимов.

Режим $3\left(\omega=3,9 c^{-1}\right)$. При повышении частоты $\omega$ система выходит за пределы области динамической неустойчивости и совершает вынужденные колебания под воздействием кинематического возбуждения. 
Режним 4. Поскольку спектр пульсации содержит также гармонику на частоте $\omega$, можно было также ожидать параметрическое возбуждение в окрестности $\omega \approx 2 p$, что и подтвердилось при численном эксперименте. В рассматриваемом режиме вынужденные колебания имеют относительно незначительную амплитуду, в то время как параметрические колебания проявляются достаточно интенсивно. Срыв нарастания колебаний происходит при резких изменениях частотного спектра в зоне упоров

Режим 1

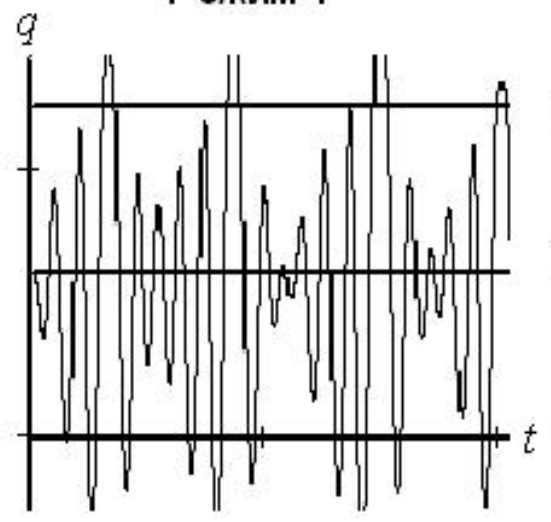

Режим 3

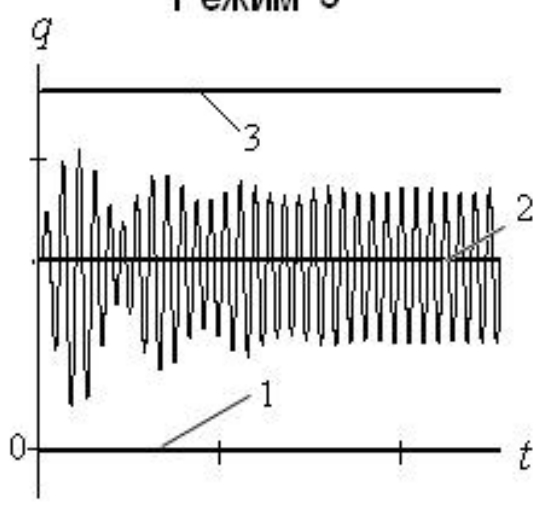

Режим 2

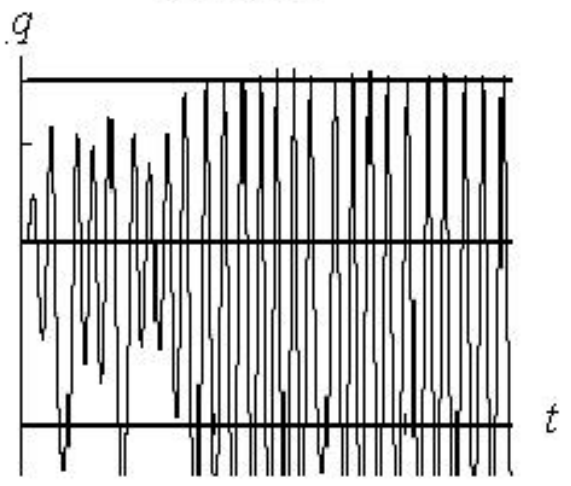

Режим 4

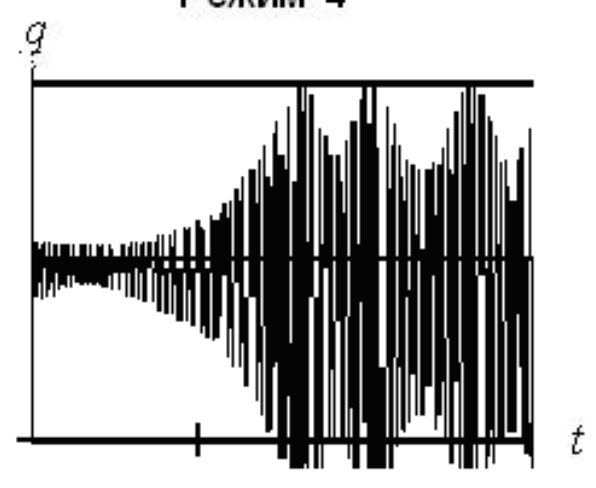

Рис. 6.4. Типовые режимы при совместном кинематическом и параметрическом возбуждении 


\section{СПИСОК ЛИТЕРАТУРЫ}

1. Бежанов Б. Н., Бушунов В. Т. Производственные машиныавтоматы.- М.-Л., Машгиз, 1973. - 368 с.

2. Бидерман В.Л. Теория механических колебаний. - М.: Высш. шк., 1980. - 408 с.

3. Вейц В.Л. Динамика машинных агрегатов. - Л.: Машиностроение, 1969. - $370 \mathrm{c}$.

4. Вибрации в технике: Справочник. - М.: Машиностроение, 1995. T.6. $-456 \mathrm{c}$.

5. Вульфсон И. И. О колебаниях систем с параметрами, зависящими от времени. Прикладная математика и механика. 1969. Т.33, №2.- С. 331-337.

6. Вульфсон И.И. Динамические расчеты цикловых машин. -Л.: Машиностроение, 1976. - 328 с.

7. Вульфсон И.И. Колебания машин с механизмами циклового действия. - Л.: Машиностроение, 1990. - 309 с.

8. Вульфсон И.И. Колебания в машинах: Учеб. пособие. для втузов. 3-ье изд. - СПб.: изд-во СПГУТД, 2008. - 260 с.

9. Вульфсон И.И. К проблеме снижения виброактивности цикловых механизмов с помощью динамически разгружателей. - Вестник научнотехнического развития. №3 (31). 2010. - С.3-13.

10. Вульфсон И.И. Динамика цикловых машин. - СПб.: Политехника, 2013. $-417 \mathrm{c}$.

11. Вульфсон И.И. Условия динамической устойчивости при совместном учёте медленных и быстрых изменений параметров. - Вестник научнотехнического развития. 2015. №3 (91) С.3-13.

12. Вульфсон И.И. К проблеме динамической взаимосвязанности электродвигателя и механического привода цикловых машин. - Теория механизмов и машин. Т.14 .№4 (32). 2016. С.173- 181.

13. Вульфсон И.И. Краткий курс теории механических колебаний / И.И. Вульфсон. - Библиотека ВНТР. - М.: ВНТР, 2017. -241 с.

14. Вульфсон И.И., Коловский М.З. Нелинейные задачи д динамики машин. - Л.: Машиностроение, 1968. - 281 с.

15. Вульфсон И. И., Тышкун А. П., Клементьев А. В. / А.С. 1117354. СССР. Привод игольницы основовязальной машины. Б. И. № 37. 1984.

16. Динамика машин и управление машинами: Справочник / В. К. Асташев, В. И. Бабицкий, И. И. Вульфсон и др. Под ред. Г. В. Крейнина - М.: Машиностроение, 1988. - 239 с. $-390 \mathrm{c}$.

17. Кобринский А. Е. Механизмы с упругими связями. М.: Наука, 1964.

18. Кожевников С. Н. Динамика машин с упругими звеньями. - Киев: АН УССР, 1961. - $160 \mathrm{c}$.

19. Коловский М. 3. Динамика машин. - Л.: Машиностроение, 1989. $264 \mathrm{c}$. 
20. Колчин Н. И. Механика машин. В 2-х т. Л.: Машиностроение, 1971, 1972.

21. Лурье А. И. Аналитическая механика.- М.: Физматгиз, 1961.- 824 с.

22. Механика машин: Учеб. пос. для втузов / И.И. Вульфсон, М.З. Коловский, Э.Е. Пейсах и др.; под ред. Г.А. Смирнова. - М.: Высш. шк., 1996. $511 \mathrm{c.}$

23. Преображенская М. В., Вульфсон И.И. Расчет колебаний при учете динамической характеристики двигателя, привода и зазоров. (Dv-Zaz) № 2014611960. 14.02.2014

24. Преображенская М. В., Вульфсон И.И. Синтез закона движения кулачкового механизма с учетом упругости толкателя (Kul_Mag). №2016613814. 17.02.2016

25. Преображенская М. В., Вульфсон И.И. Динамика цикловых механизмов с нестационарными связями. (Mag01). № 2016613815. 06.04.2016.

26. Преображенская М. В., Грибкова Т. С. Проверочная программа по курсовому проектированию (раздел: синтез закона движения толкателя кулачкого механизма) (Kinst_prow). № 2014663122. 16.12.2014.

27. Преображенская М. В., Грибкова Т. С. Проверочная программа для курсовой работы по теории колебаний (раздел: изгибные колебания) (TK_prow). № 2014662838. 10.12.2014.

28. Ротбарт Г. А. Кулачковые механизмы. - Л.: Изд-во судостр. пром., 1960. $-336 \mathrm{c}$.

29. Семенов Ю. А. Механика. Теория механических колебаний: учеб. пособие. - СПб.: Изд-во Политехн. ун-та, 2008. - 412 с.

30. Теория механизмов и машин: Учеб. пос. для втузов / М.3. Коловский, А. Н. Евграфов, Ю. А. Семенов и др.; 2-е изд., испр. - М.: Изд. центр «Академия»., 2008. - 560 с.

31. Теория механизмов и машин: Учеб. для втузов / К. В. Фролов, С. А. Попов, А. К. Мусатов и. др.; под ред. К.В. Фролова. 5-е изд. - М.: Высш. шк., 2005. - 496 c.

32. Тир К. В. Механика полиграфических автоматов. - М.: Книга, 1960. $-496 \mathrm{c}$.

33. Фреман Н., Фреман П. У. ВКБ-приближение. - М.: Мир, 1967.- 168 c.

34. Advanced theory of mechanisms and machines/ M. Z. Kolovsky, A. E. Evgrafov, Yu. A. Semenov, A. V. Slouch. - Berlin, Heidelberg: Springer, 2000. 394 p.

35. Beitelschmitd M., Dresig H. Machinendynamic. - Aufgaben und Beispiele. - Berlin, Heidelberg: Springer, 2015. - 407 S.

36. Dresig H., Vulfson I. I. Dynamik der Mehanismen. - Wien, New York: Spinger, 1989. - 318 S. 


\section{ПРИЛОЖЕНИЕ}

\section{Синтез закона движения кулачкового механизма с учетом упругости толкателя (Программа "Kul_mag")}

Программа [24] предназначена для синтеза закона движения кулачкового механизма с учетом упругости толкателя. Система представлена в виде динамической модели с одной степенью свободы с переменной передаточной функцией, осуществляемой кулачковым механизмом. Для синтеза закона движения толкателя используется метод безразмерных характеристических функций. Программа интерактивна. Она позволяет в режиме диалога, наблюдая графики динамических процессов, протекающих в приводе, с помощью мыши и кнопок управлять ими, изменяя динамические параметры системы и структурные параметры законов движения. Таким образом, можно непосредственно на экране монитора оптимизировать законы движения, и динамические параметры привода.

Функции кнопок:

1 - вывод на экран программного закона движения.

2 [^] - вывод динамической модели.

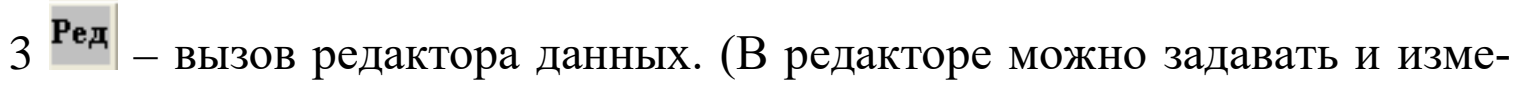
нять исходные данные.)

При нажатии этой кнопки открывается окно:

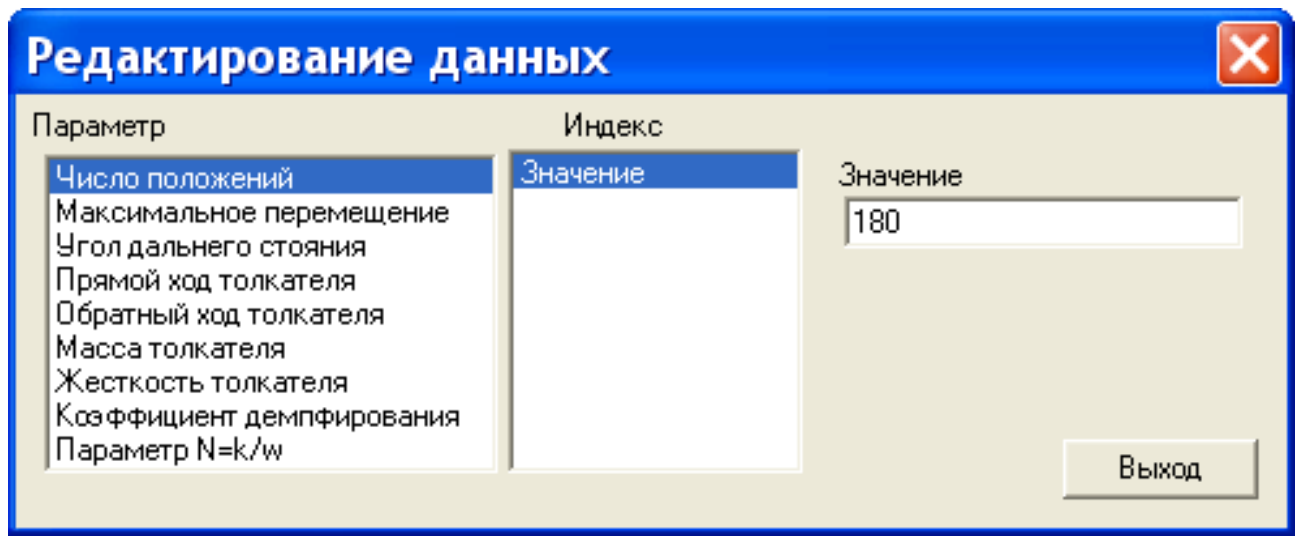

Рис. П.1. Окно ввода и редактирования данных

4 奜 - на экран выводится график закона движения с учетом упругости толкателя при нулевых начальных условиях.

5 奜2 - тот же график, но для установившегося движения. q(0)

6 q(0) - начальные условия для установившегося движения.

7 舀 - открывается окно, предназначенное для изменения массы, жесткости толкателя и коэффициента демпфирования: 


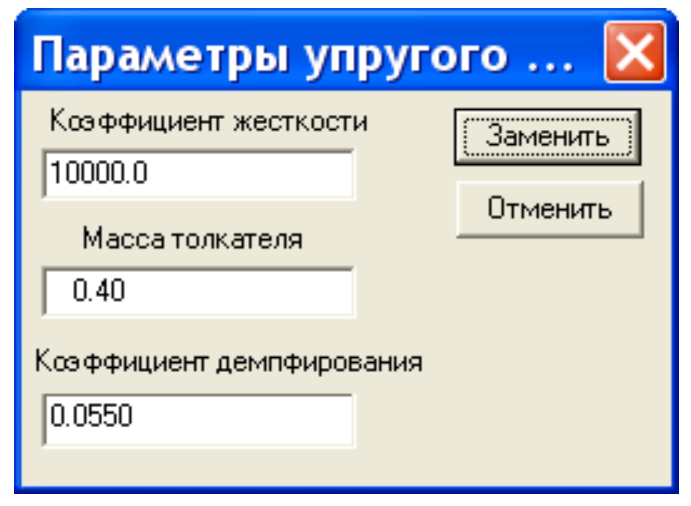

Рис. П.2. Ввод и редактирования параметров упругого толкателя

8 \#

— при нажатии этой кнопки на графике появляются деления по оси $\varphi$ и три активных символа: $\Pi, \Pi^{\prime}$ и $\Pi^{\prime \prime}$. При щелчке мыши на одном из этих символов на графике появляются деления для соответствующей функции. При повторном нажатии кнопки 8 сетка исчезает.

$9+$ и $10-$ - увеличение или уменьшение активного параметра. Активными могут стать параметры, изображенные на экране серым цветом. При наведении на них курсора меняется его форма, а при щелчке мыши изменяется цвет надписи. При этом параметр становится активным.

11 - возврат к исходным данным. При нажатии этой кнопки программа возвращается к данным, сохраненным в используемом файле данных. Кнопкой рекомендуется пользоваться в случаях, когда в процессе работы параметры менялись, но не сохранялись в файле данных.

12 基 - вывод и удаление с экрана параметров закона движения. По умолчанию все параметры выводятся в правой верхней части экрана.

13 - вывод информации.

14 - сохранение данных. При нажатии кнопки открывается стандартное окно для выбора имени файла. Данные могут быть сохранены и в старом, и в новом файле с произвольным именем. Чтобы данные загружались по умолчанию, их надо сохранять в файле Kul.dat.

15 - ввод данных из выбранного файла. Открывается стандартное окно для выбора имени файла данных.

16

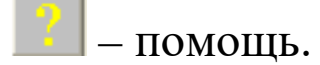

При запуске программы данные вводятся по умолчанию из файла данных Kul.dat, помещенного в одной директории с выполняемым файлом Kul_mag.exe. Если файл Kul.dat отсутствует, или в нем нет данных, то на экране появится пустое поле с нулевыми параметрами закона движения в правой верхней части экрана. Чтобы задать данные, надо нажать кнопку 2 - Ред, и в открывшемся окне редактора ввести данные. Если желательно, чтобы в 
дальнейшем данные открывались по умолчанию, без вызова файла данных, то их надо сохранить в файле Kul.dat, но при этом надо иметь в виду, то следующий пользователь может изменить эти файлы, поэтому, если предполагается неоднократное использование программы, то нужно создавать индивидуальные файлы данных.

Изменение данных можно производить не только в редакторе данных, но и с помощью активных надписей. Все надписи, на которых меняется форма курсора со стрелки на «указующий перст» могут быть активированы, для чего следует щелкнуть на них левой кнопкой мыши. При этом меняется цвет надписи. При щелчке правой кнопки мыши надпись возвращается в исходное состояние. Если надпись активна, то с помощью кнопок $<+>$ и $<->$ можно менять значения параметров. Кроме того, с помощью мыши можно сдвигать границы участков на графике. Для этого надо «поймать» мышью нужную границу, (при этом форма курсора меняется на горизонтальные стрелки) и передвигать её вправо или влево. При этом происходит полный пересчет графика.

На рис. П.З. представлено главное окно программы, в верхнем правом углу которого представлены все константы закона движения, в центре экрана изображен закон колебаний модели с учетом упругости толкателя. В нижней части окна справа и слева выведены параметры, которые можно активировать для дальнейшего варьирования.

Во время демонстрации вынужденных колебаний прямо на экране можно менять закон движения и наблюдать за влиянием изменения закона движения на вынужденные колебания. Для этого надо «захватить» мышью вертикальные линии закона движения и перемещать их вправо, или влево, изменяя длины участков разбега, постоянной скорости, или выбега.

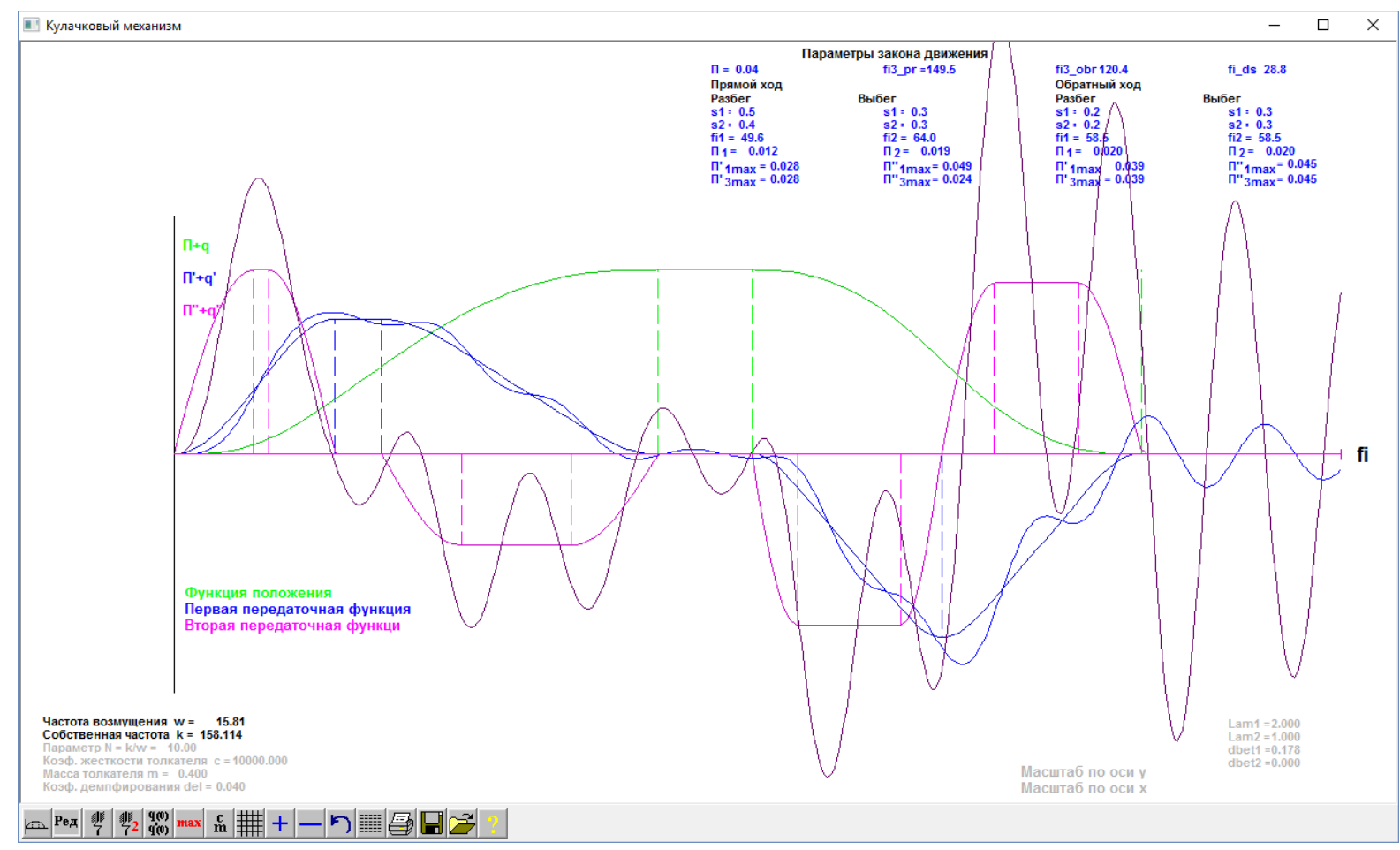

Рис. П.З. Колебания, вызванные кинематическим возбуждением 
Кроме того, после активизации надписей, расположенных в правой нижней части экрана, с помощью кнопок <+> и <-> можно менять параметры модели и исследовать их влияние на вынужденные колебания модели. 


\section{I.I. Vulfson, T. S. Gribkova, M. V. Preobrazhenskaya}

\section{Program laws of technological machines actuators}

\section{ABSTRACT}

The book gives basic concepts about the modern approach to solving an important engineering problem - the synthesis of the law of motion for the actuators of mechanisms at solving modern problems of designing high-speed machines, taking into account technological and dynamic requirements. Particular attention is paid to methods of suppressing excited oscillations, which are the main source of distortions of given program motions. When solving optimization problems, an apparatus of dimensionless characteristic functions is used that facilitates the variation and optimization of the parameters. Appropriate computer simulation programs are provided, the use of which implements a rational combination of analytical and numerical methods.

The book significantly reduces the complexity and labor intensity of calculations and can serve as a tool for mechanical engineers. It can also be used as a teaching aid for students, masters and for the upgrading of teachers of the relevant specialties.

Printed in authors' edition.

\section{The library of the Journal "Bulletin of Science and}

\section{Technical Development"}

(http://www.vntr.ru)

Vulfson, I. I., Gribkova T. S., Preobrazhenskaya M. V. Short course of the vibrations theory- Moscow: VNTR, 2017. — 108 p.p.

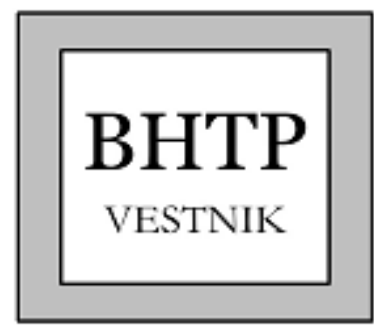

Moscow 


\section{Сетевое издание}

Иосиф Исаакович Вульфсон,

Тамара Степановна Грибкова

Марианна Васильевна Преображенская

\section{ПРОГРАММНЫЕ ЗАКОНЫ ДВИЖЕНИЯ РАБОЧИХ ОРГАНОВ ТЕХНОЛОГИЧЕСКИХ МАШИН}

Файл книги подготовлен автором при участии редакции журнала «Вестник Научно-Технического Развития»

Библиотека журнала «Вестник Научно-Технического Развития» (http://www.vntr.ru)

\section{BHTP}

VESTNIK

Москва, 2017 